National Bureau of Stenda"dy

\title{
Activation Analysis Section:
}

\author{
Summary of Activities \\ July 1969 to June 1970
}

U.S.

ARTMENT

OF

JMMERCE

National

Bureau

of

landards 



\section{NBS TECHNICAL NOTE 548 ISSUED DECEMBER 1970}

Nat. Bur. Stand. (U.S.), Tech. Note 548, 164 pages (Dec. 1970) CODEN: NBTNA

\section{Activation Analysis Section: Summary of Activities, July 1969 to June 1970}

Philip D. LaFleur and Donald A. Becker, Editors

Activation Analysis Section

Analytical Chemistry Division Institute for Materials Research

National Bureau of Standards

Washington, D.C. 20234

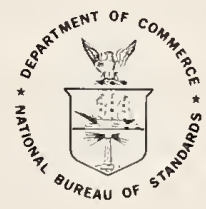

NBS Technicai Notes are designed to supplement the Bureau's regular publications program. They provide a means for making available scientific data that are of transient or limited interest. Technical Notes may be listed or referred to in the open literature.

For sale by the Superintendent of Documents, U.S. Government Printing Office, Washington, D.C., 20402. (Order by SD Catalog No. C 13.46:548). Price \$1.25. 
The Analytical Chemistry Division was established as a separate division at the National Bureau of Standards on September 1, 1963, and became part of the Institute for Materials Research in the February 1, 1964, reorganization. It consists at present of nine sections and about 100 technical personnel encompassing some 60 different analytical competences from activation analysis and atomic absorption to vacuum fusion and $\mathrm{x}$-ray spectroscopy. These competences, and in turn the sections which they comprise, are charged with research at the forefront of analysis as well as awareness of the practical sample, be it standard reference material or service analysis. In addition it is their responsibility to inform others of their efforts.

Formal publication in scientific periodicals is a highly important output of our laboratories. In addition, however, it has been our experience that informal, annual summaries of progress describing efforts of the past year can be very valuable in disseminating information about our programs. A word is perhaps in order about the philosophy of these yearly progress reports. In any research program a large amount of information is obtained and techniques developed which never find their way into the literature. This includes the "negative results" which are so disappointing and unspectacular but which can often save others considerable work. Of importance also are the numerous small items which are often explored in a few days and which are not important enough to warrant publication--yet can be of great interest and use to specialists in a given area. Finally there are the experimental techniques and procedures, the designs and modifications of equipment, etc., which often require months to perfect and yet all too often must be covered in only a line or two of a journal article. 
Thus our progress reports endeavor to present this information which we have struggled to obtain and which we feel might be of some help to others. Certain areas which it appears will not be treated fully in regular publications are considered in some detail here. Other results which are being written up for publication in the journal literature are covered in a much more abbreviated form.

At the National Bureau of Standards publications such as these fit logically into the category of a Technical Note. In 1970 we plan to issue these summaries for all of our sections. The following is the fourth annual report on progress of the Activation Analysis Section.

W. Wayne Meinke, Chief Analytical Chemistry Division 
The Activation Analysis Section has the responsibility to develop and apply measurement techniques for the quantitative and qualitative analysis of materials through the use of nuclear reactions.

The basic activities of the Section may be divided into two general areas, the research and development area and the sample area. The section has a very real responsibility for the development of new techniques, and the refinement of existing techniques, to increase the applicability of activation analysis and to reduce the cost of analysis to become more competitive with non-nuclear methods of analysis.

A very important part of the section's activities is involved in the certification of Standard Reference Materials in cooperation with the other Sections in the Analytical Chemistry Division. The Office of Standard Reference Materials of the National Bureau of Standards is currently developing, or has issued, Standard Reference Materials of very high purity which are well characterized for their trace element content. The Activation Analysis Section has made a significant contribution in the certification of these standard materials.

Activation analysis is particularly suited for the analysis of trace and ultra-trace components of materials because of the very high sensitivity for many elements, the lack of a reagent "blank" in most analyses, and the opportunity for reasonable accuracy when carefully done.

The Activation Analysis Section is composed of several groups, three of which are oriented to a specific source of nuclear radiation: the reactor, IINAC, and Cockcroft-Walton generator groups; a group for the development of radiochemical separations and a project oriented to the analysis of biological materials; a nuclear data handling project and project for the utilization of the nuclear track technique 
for quantitative analysis. Considerable interaction among the groups is encouraged so that activities of common interest are worked upon jointly in the several groups.

A roster of the personnel in the section is given in Part 7. In the past the section has enjoyed the presence of scientists from abroad, and from within the United States; they have contributed to a very stimulating environment. It is hoped that we may continue to utilize this program in the future.

In order to specify adequately procedures, it has been necessary occasionally to identify commercial materials and equipment in this report. In no case does such identification imply recommendation or endorsement by the National Bureau of Standards, nor does it imply that the material or equipment identified is necessarily the best available for the purpose.

Philip D. LaFleur, Chief Activation Analysis Section Analytical Chemistry Division 
1. INTRODUCTION. . . . . . . . . . . . . . . . . 2

2. LIAISON . . . . . . . . . . . . . . . 4

3. ACTIVATION ANALYSIS WITH THE NUCLEAR REACTOR. • • 5

A. Introduction. . . . . . . . . . . . 5

B. Facilities. . . . . . . . . . . . 6

1. Characterization of the NBSR for Neutron Activation Analysis . . . . . . . . . . .

2. Installation and Operation of a ComptonSuppressed Gamma Scintillation Spectrometer............. . 23

C. Research Activities . . . . . . . . . 28

1. Characterization of Medieval Glass by Neutron Activation Analysis . . . . . . 28

2. The Determination of Sulfur Using Secondary Reactor Tritons . . . . . . . . . 37

3. Analysis for Iodine in Protein Solutions by Neutron Activation Analysis. . . . . . 41

4. Lead Alloy Flux Monitors for Neutron Activation Analysis... . . . . . . 42

5. Extraction of Metals with Bis(2-ethylhexyl) Orthophosphoric Acid. . . . . . 44

6. Determination of Short-lived Trace Impurities in Sodium Metal by Neutron Activation Analysis... . . . . . 48

D. Analysis of Standard Reference Materials. . 50

1. Biological Standard Reference Materials: Orchard Leaves. . . . . . . . . 50

2. Determination of Cobalt, Silver, and Gold in SRM Glass by Neutron Activation Analysis. . . . . . . . . . . 56

3. Survey of SRM $20 \mathrm{ppb}$ Trace Elements . . 60

4. Survey of SRM $i$ ppm Trace Elements in $\dot{G}$ iass 61

5. The Determination of Manganese in Trace Elements in Glass Samples.... 
6. Intermediate Purity Sulfur. . . . . . . 63

7. Potassium Chloride Single Crystal. . . 65

8. Determination of Indium and Copper in SRM High Purity Gold Wire by Neutron Activation Analysis . . . . . . . . . 66

9. The Determination of Cobalt in CobaltAluminum Flux Wires... . . . . . 69

10. Analysis of Cast Iron Steels for

11. Activation Analysis of SRM Steels Using Group Radiochemical Separations . . . . . 72

12. The Determination of Cobalt in SRM Steels by Neutron Activation Analysis. . . . . 74

13. Determination of Copper and Cobalt in SRM

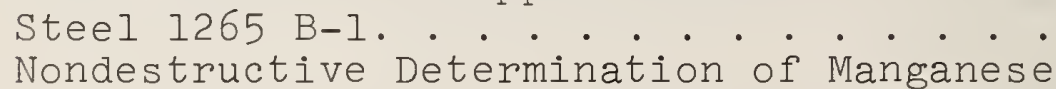

14. Nondestructive Determination of Manganes
and Vanadium in Iron and Steel Standard Reference Materials . . . . . . . 76

15. The Multielement Analysis of SRM Steels 1261 and 361 by Destructive Neutron Activation Analysis... . . . . . 78

E. Special Analyses. . . . . . . . . . . 79

1. Determination of Copper Homogeneity in Copper-Lead Alloy. . . . . . . . . .

2. Analysis of Neodymium Laser Glass by Neutron Activation Analys is . . . . . . 80

3. Determination of Trace Elements in Ruby Laser Crystals. . . . . . . . . . 82

4. Determination of Trace Elements in Synthetic Sapphire Crystals . . . . . 87

5. Determination of Gold in Semiconductor Silicon by Neutron Activation Analysis.

F. The Nuclear Track Technique . . . . . . . 88

1. Introduction. . . . . . . . . . . . . 88

2. Analysis of Uranium and Boron in Trace Elements in Glass... . . . . 89

3. Analysis of Orchard Leaves for Boron and Uranium . . . . . . . . . . . . . 94

4. Photo-fission Studies . . . . . . 94 
4. ACTIVATION ANALYSIS WITH THE COCKCROFT-WALTON NEUTRON GENERATOR

A. Introduction. . . . . . . . . . . 96

B. Research Activities . . . . . . . . 97

1. Gamma-ray Spectra and Experimental Sensitivities for 3-MeV Neutron Activation Analysis. . . . . . . . . . . . 97

2. D-D Neutron Activation Interferences in 14-MeV Neutron Activation Analysis. . . . 99

3. Thermalization Characteristics of $3-\mathrm{MeV}$ Neutrons............ 100

4. Pulse Pile-up and Nuclear Interferences in the Analysis for Trace Oxygen. . . . 103

C. Analysis of Standard Reference Materials

1. Cadmium Cyclohexane Butyrate SRM 1053a. . 105 2. Magnesium Cyclohexane Butyrate SRM 1061c. 105

D. Special Analyses

1. Oxygen in Metallic Barium . . . . . . . 106

2. Oxygen in Niobium Metal and Niobium-
Titanium Alloy. . . . . . 106

E. Status of Neutron Generator Facility

1. Reconditioning the Penning Ion Source. . 107

2. Health Physics........... 107

5. ACTIVATION ANALYSIS WITH THE NBS LINAC

A. Facilities. . . . . . . . . . . . 109

1. Target System . . . . . . . . . 109

2. Sample Terminal . . . . . . . . . . 111

3. Beam Dump . . . . . . . . . . . . . . 111

4. Pneumatic Transfer and Shuttle Systems. . 111

5. LINAC Interface System. . . . . . . . . 113

6. Pneumatic Tube Coupled Lazy Susan . . . 114

B. Analyses

1. Determination of Thallium in Glass and Zinc Using Photon Activation. . . . . . 
Page

2. Determination of Gold, Tantalum, Manganese and Iron in Cadmium Metal by Photon Activation .......... . 120

3. Determination of Nitrogen in Metals by Photon Activation . . . . . . . . 122

6. APPLICATION OF COMPUTERS TO ACTIVATION ANALYSIS

A. Introduction. . . . . . . . . . . . 124

B. ALSPIS - Activation Analysis Peak Area Program . . . . . . . . . . . 128

C. A Mini-Computer and the Activation Analysis Section . . . . . . . . . . . 135

D. Miscellaneous . . . . . . . . . . 139

7. PERSONNEL AND ACTIVITIES

A. Personnel Listing... . . . . . . . 140

B. Publications. . . . . . . . . . . 141

C. List of Talks. . . . . . . . . . . 142

8. ACKNOWLEDGMENTS . . . . . . . . . . . 145

9. REFERENCES. . . . . . . . . . . . 146 
1. Positions of the pneumatic tube terminals in the NBSR . . . . . . . . . . . . . 7

2. Neutron cross sections of copper, gold and cobalt as a function of neutron energy. . . . 8

3. Diagramatic representation of the circumferential flux distribution in the NBSR pneumatic tubes at three positions inside the rabbit.

4. Copper foil placement for circumferential flux measurements in the NBSR pneumatic tubes. . .

5. Axial flux distribution in the NBSR pneumatic tubes.......... . . . . . . . 12

6. Axial flux dropoff inside the rabbits (normalized to proximal end $\equiv l .00$ for each pneumatic tube )

7. Device used to measure pressures generated from irradiated samples.

8. Vertical distribution of the total neutron flux in the NBSR LTIF. . . . . . . . . . . . . 21

9. Partially shielded Compton-suppressed gammaray scintillation spectrometer with associated electronics.

10. Schematic of the Compton-suppressed gamma-gamma scintillation spectrometer. . . . . . . . 25

11. Gamma sum spectra of cobalt-60. . . . . . 26

12. Gamma-gamma coincidence spectra of cobalt-60. . 27

13. Gamma-gamma coincidence spectra of cesium-137 . 28

14. Fragment of Medieval glass with sample cut from one edge. . . . . . . . . . . . . . . . . 33

15. French 14th Century circle of stained glass Angel of the Passion.

16. Correlation between weight and neutron selfabsorption of lithium fluoride. 
17. Lead alloy ingot being rolled into a thin foil. 43

18. Extraction curves for bis(2-ethylhexyl)

orthophosphoric acid as a function of hydrochloric acid concentration

19. Extraction curves for bis(2-ethylhexyl) orthophosphoric acid as a function of perchloric acid concentration

20. Extraction curves for bis(2-ethylhexyl) orthophosphoric acid as a function of nitric acid concentration.

21. Freeze drying apparatus for the removal of moisture from biological samples. . . . . .

22. Ruby crystal as cut for analysis. . . . . . 83

23. Microscope display for fission track counting. 90

24. Boron distribution across the diameter of a glass wafer. . . . . . . . . . . 92

25. Photon activation study depicting uranium weight fraction to total track response.

26. 3-MeV neutron buildup as a function of time at constant beam current........... 100

27. Relative flux determinations as a function of moderator thickness . . . . . . . . . 102

28. LINAC irradiation assembly. . . . . . . . . 110

29. LINAC target assembly . . . . . . . . . . 110

30. LINAC beam dump. . . . . . . . . . . 112

31. LINAC pneumatic transfer system control . . . 112

32. LINAC interface system control. . . . . . . 113

33. Varian 622/i minicomputer . . . . . . . 136 
1. NBSR irradiation facilities

2. Experimental neutron flux - NBSR pneumatic facilities

3. Circumferential flux distribution in NBSR pneumatic tube facilities

4. Cadmium ratios for the NBSR pneumatic tube facility.

5. Radiation damage and temperature measurements at 10 MW in RT-1..............

6. Radiation damage and temperature measurements at $10 \mathrm{MW}$ in RT-2. . . . . . . . . . . .

7. Radiation damage and temperature measurements at 10 MW in RT-3...............

8. Radiation damage and temperature measurements at 10 MW in RT-4.............

9. Heat resistance of polyethylene rabbits: effect of irradiation time . . . . . . . . . . . . .

10. Pressure measurements on neutron irradiated. samples

11. Cadmium ratios in the LTIF. . . . . . . . . 22

12. Circumferential flux variation in the NBSR LTIF irradiation facility. . . . . . . . . . . . .

13. Results of activation analysis of simulated. ancient glasses... . . . . . . . . . .

14. Homogeneity of Medieval and 18th Century glasses.

15. Results of analysis of Bourges glasses. . . .

16. Alkali ratios found in Medieval glasses.... .

17. Manganese/sodium ratios found in Medieval glasses 
18. Results of activation analysis of Medieval

window.

19. Results of sulfur analysis.

20. NBS biological Standard Reference Materials .

21. Moisture removal for orchard leaf samples

22. Manganese homogeneity: orchard leaves. . . . 54

23. Manganese homogeneity: orchard leaves. . . . 55

24. Combined averages for all containers. . . . . 56

25. Results of analysis of 1 ppm glass (SRM 615). . 58

26. Results of analysis of 50 ppm glass (SRM 613). 59

27. Results of analysis of 500 ppm glass (SRM 611). 59

28. Glass impurities. . . . . . . . . . 6 60

29. Analytical results for 1 ppm glass. . . . . . 61

30. Manganese concentrations in trace elements in

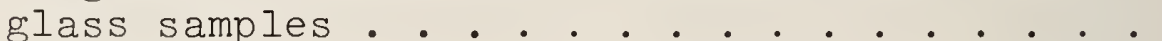

31. Impurities found in intermediate purity sulfur. 64

32. Variation in impurities found - surface vs. interior of sample. . . . . . . . . . 65

33. Analyses for copper and indium in SRM gold wire 69

34. Analysis results for cobalt in cobalt-aluminum wire.

35. Results of tellurium analysis in SRM cast iron steel

36. Results of analysis of SRMs 1263, 1264, 1265..

37. Results of analysis of SRM ductile irons. . . 73

38. Results of analysis for cobalt. . . . . . . 75 


\section{LIST OF TABLES (cont)}

$\underline{\text { Table }}$

$\underline{\text { Page }}$

39. Results of activation analysis of SRM 1265 B-1. 76

40. Results of manganese and vanadium analyses of iron and steel Standard Reference Materials. .

41. Results of multielement analysis of SRM steels 1261 and 361 .

42. Results of activation analysis of neodymium glasses

43. Results of activation analysis of ruby crystals, group I. . . . . . . . . . . .

44. Results of activation analysis of ruby crystals, group II. . . . . . . . . . . .

45. Results of activation analysis of ruby crystals, group III.

46. Detection limits for trace elements in ruby.

47. Trace elements observed in sapphire crystals. .

48. Results of analysis of silicon wafers......

49. Boron and uranium content in the trace elements in glass (50 ppm).

50. Boron and uranium content in the trace elements in glass

( $1 \mathrm{ppm}$ )

51. Boron and uranium content in the trace elements in glass $(0.02)$.

52. Experimental sensitivity for 3-MeV neutron activation analysis

53. Results of trace oxygen interferences . . . . 104

54. Results of analyses for thallium in NBS glass . 117

55. Thallium content in zinc and zinc salts... . 118

56. Representation of alphanumeric character string internally and as base ten numbers. . . . . . 

ACTIVATION ANALYSIS: SUMMARY OF ACTIVITIES

\section{July 1969 to June 1970}

Edited by

Philip D. LaFleur and Donald A. Becker

This survey of the activities of the Activation Analysis Section covers the period July 1969 to June 1970. An overview of the progress made by the various projects in the section is given. A discussion of the operating characteristics of the NBS Reactor for activation analysis is given and the research activities of the various projects are discussed. Special attention is given to the many practical problems encountered by the section during the year, and the results of the solutions to these problems, especially the analysis of Trace Elements in Glass Standard Reference Material (SRM) and of the botanical SRM. The quantitative analysis for boron using the nuclear track technique is discussed as well as extension of the anlaysis for uranium using this technique.

An extensive discussion of the value of $3-\mathrm{MeV}$ neutrons in neutron generator activation analysis and the experimental parameters for using these neutrons is discussed. The new target and pneumatic transfer systems at the NBS LINAC for photon activation analysis are described and the application of photon activation analysis for determining thallium in glass and high purity metals and gold, manganese and iron in high purity cadmium is discussed. Research into determination of nitrogen by photon activation analysis is described.

A computer program, ALSPIS, for peak finding and integration is described and the application of a mini-computer to the Activation Analysis Section is noted.

Key words: activation analysis; Cockcroft-Walton neutron generator; computer programming; NBS IINAC; NBS Reactor; Standard Reference Materials. 
During the past year the Activation Analysis Section has reached a turning point. For the past several years we have been engaged in demonstrating our capabilities for analysis both within and outside of the National Bureau of Standards. We now feel that we have satisfactorily demonstrated this capability and intend during the coming year to spend considerably more of our time on research activities and the development of new analytical techniques.

As was mentioned in last year's progress report, our most serious problem at that time was the attainment of a capability using computers for processing data. The addition of Dr. H. P. Yule to our staff has started us on the solution to this problem and we are looking forward, in the next several years, to the fruitful application of automated data processing both on large digital computers and on mini computers.

During this year the NBS Reactor has operated all year at a power level of 10 Megawatts. The operating schedule has been approximately three to four weeks at full power and then about three days of down time for routine maintenance. The flight lines in the four originally installed pneumatic tube systems have been replaced with armoured polyethylene tubing and all four systems are operative and are being fully characterized. The pneumatic tube system in the thermal column facility should be installed very early in fiscal year 1971 as should the 7/8 in. pneumatic tube system.

A comprehensive study of the application of $3-\mathrm{MeV}$ neutrons from neutron generators and of the problems of $3-\mathrm{MeV}$ neutron buildup in 14-MeV activation analysis and of the use of moderated 3-MeV neutrons has been completed. This was the final phase of the research with the neutron generator laboratory and the application of this machine in the near future will be primarily in the analysis of samples. 
During this year a new bremsstrahlung converter and rabbit system has been installed in the NBS LINAC. This has caused the facility to be down during the last four months of the year, however, the new system should be operable in August of 1970. Work has been completed on the analysis of carbon in a number of high purity metals using the LINAC, and efforts have begun on the analysis of elements other than the light elements in a number of sample matrices. 
During this year we have enjoyed a fruitful cooperative program with the Smithsonian Institution and Mrs. Jacqueline Olin of that organization. This program has resulted in some very interesting data on the characterization of stained glass, so that future work may be extended for the elucidation of the origin and/or period of manufacture of stained glass windows.

Fledgling cooperative programs have also begun with the Internal Revenue Service of the Treasury Department, in this case with Mr. C. Michael Hoffman and Mr. Maynard Pro of that laboratory, in the analysis of bullet lead. We are expecting to become more deeply involved in forensic science and are looking forward to an interesting and fruitful collaboration with the Treasury Department.

With the occasion of the closing down of the Naval Research Laboratory Reactor, we have been participating with the Reactor Radiation Division with accomodating new users to the NBS Reactor and expect to have many encounters with these new users during the next year. We anticipate additional cooperative programs may result from these contacts. It is our opinion that the cross-fertilization resulting from activities of this kind is beneficial to both groups who participate, and we welcome inquiries about the use of NBS facilities either on a cooperative basis, or where the interested party merely wishes to utilize the radiation source. 
3. ACTIVATION ANALYSIS WITH THE NUCLEAR REACTOR

A. Introduction

During this year we have continued our efforts to attain a high degree of versatility in activation analysis and have spent much of our time in developing a greater expertise in nondestructive activation analysis, particularly coupled with computer data reduction. We intend, of course, to continue our efforts in obtaining the highest possible versatility in both destructive and nondestructive activation analysis and in the investigation of parameters affecting accuracy and precision in activation analysis.

We have spent a great deal of time in characterizing the two pneumatic tube facilities which became available this year. All four of the originally installed facilities are now available and have been very carefully studied in terms of temperature effects, relative neutron fluxes and to a more limited extent on neutron energy spectra. We have maintained a very satisfactory relationship with the Reactor Operations Section and with the Health Physics Section.

A greater portion of our activities this year than in past years has been spent in service analyses for organizations outside the Bureau of Standards and for other groups within the Bureau of standards. Particularly notable are our efforts in the analysis of lasers and silicon chips. We are presently beginning a service analysis program for the Department of Agriculture, involving the determination of mercury in wheat and peas and with the National Institutes of Health in the determination of iodine in blood serum samples. It is our hope that contacts with other governmental organizations in the Washington area, such as these, may continue and that interesting cooperative research programs may develop therefrom. 
1. Characterization of the NBSR for Neutron Activation Analysis

Since the last report [1] much additional information has been obtained on the irradiation characteristics of the NBSR. This information has included thermal neutron flux variations within the irradiation areas, neutron energy distribution in the various irradiation positions, temperature effects, and measurements of the gas production from several different biological materials. These different types of measurements will be treated separately here, with the techniques used presented along with the results obtained. For comparative purposes, all results obtained to date will be included here.

The current status of the NBSR irradiation facilities are given in table 1 . The operational facilities include

Table 1. NBSR irradiation facilities.

Pneumatic Facilities

\begin{tabular}{|c|c|c|c|c|}
\hline Ident. & Position & Attitude & $\begin{array}{c}\text { Access } \\
\text { Diameter }\end{array}$ & Status \\
\hline $\mathrm{RT}-1$ & Core gap edge & Horiz. & $1.5 \mathrm{in.}$ & Operational \\
\hline $\mathrm{RT}-2$ & In core & Horiz. & $1.5 \mathrm{in}$. & Operational \\
\hline $\mathrm{RT}-3$ & Core gap, $\mathrm{D}_{2} \mathrm{O}$ & Horiz. & $1.5 \mathrm{in.}$ & Operational \\
\hline $\mathrm{RT}-4$ & $\mathrm{D}_{2} \mathrm{O}$ reflector & Horiz. & 1.5 in. & Operational \\
\hline $\mathrm{RT}-\mathrm{V}$ & Vertical thimble & Vert. & 0.75 in. & Under const \\
\hline $\mathrm{RT}-\mathrm{C}$ & [Vertical thimble] & JVert. & 0.75 in. & Under develc \\
\hline$(R T-V-2)\}$ & {$[$ (Converter) $\mathrm{J}-4$} & Vert. & 0.75 in. & Under const \\
\hline $\mathrm{RT}-\mathrm{T}$ & Thermal column & $\mathrm{Ve}$ & $1.5 \mathrm{in.}$ & Under const \\
\hline \multicolumn{4}{|c|}{ Manual Access Facilities } & $*$ \\
\hline $\begin{array}{r}\text { Verti } \\
\text { thim }\end{array}$ & In core, $\mathrm{G}-2$ & Vert. & $1.5 \mathrm{in.}$ & Operational \\
\hline Vertic & Reflector, $V-4$ & Vert. & 1 in. & Planning \\
\hline
\end{tabular}


the four pneumatic tubes ( $f i g .1$ ) and the manually accessed Long Term Irradiation Facility (LTIF) located in the G-2 $\mathrm{V}$ vertical thimble. The program of characterization has included measurements on all of these operational facilities, but with particular emphasis on the pneumatic facilities which are used for the majority of the activation analysis irradiations.

a. Pneumatic Tube Irradiation Facilities

The four operational pneumatic tube facilities have diameters of $1 \mathrm{l} / 2$ inches, are powered by $\mathrm{CO}_{2}$, and terminate in significantly different neutron environments (fig. I).

RT - 1

$\mathrm{RT}-2$
TANK WALL $\rightarrow$
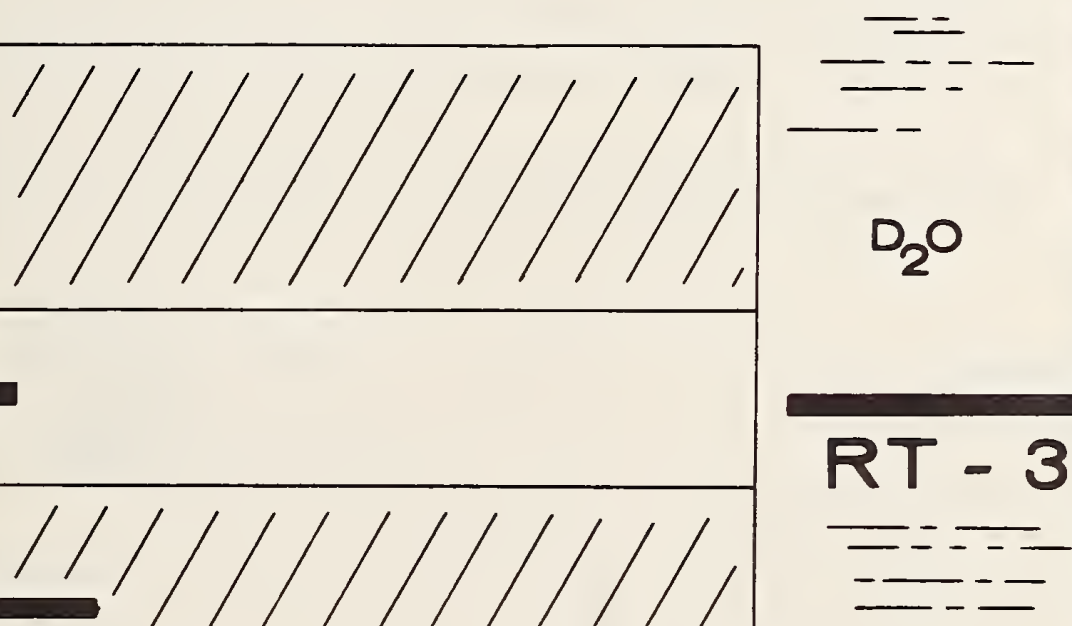

RT - 4

Figure 1. Positions of the pneumatic tube terminals in the NBSR. (Terminology: The proximal end of the rabbit is that end closest to the reactor core vertical centerline; the distal end is that furthest from the core centerline.) 
Linear polyethylene rabbits, which have a capacity of approximately $42 \mathrm{~cm}^{3}(2.6 \mathrm{~cm}$ dia $x 8 \mathrm{~cm}$ long) are used in these facilities $[1]$.

(1) Neutron Flux Measurements

Initial measurements in the pneumatic tubes were for the thermal neutron flux. Of interest was the absolute thermal neutron flux, and the variation of this neutron flux within each irradiation position. Some measurements also have been made of the neutron energy distribution characteristics within each facility.

(2) Thermal (Sub-cadmium) Neutron FIux

The thermal (sub-cadmium) neutron flux measurements were made using the thin foil technique [2]. Two different detector foils, gold and copper, were used for these measurements. Copper foils were preferred, due to the absence of significant resonance peaks in its neutron cross section (fig. 2). Some measurements were also made on gold foils,

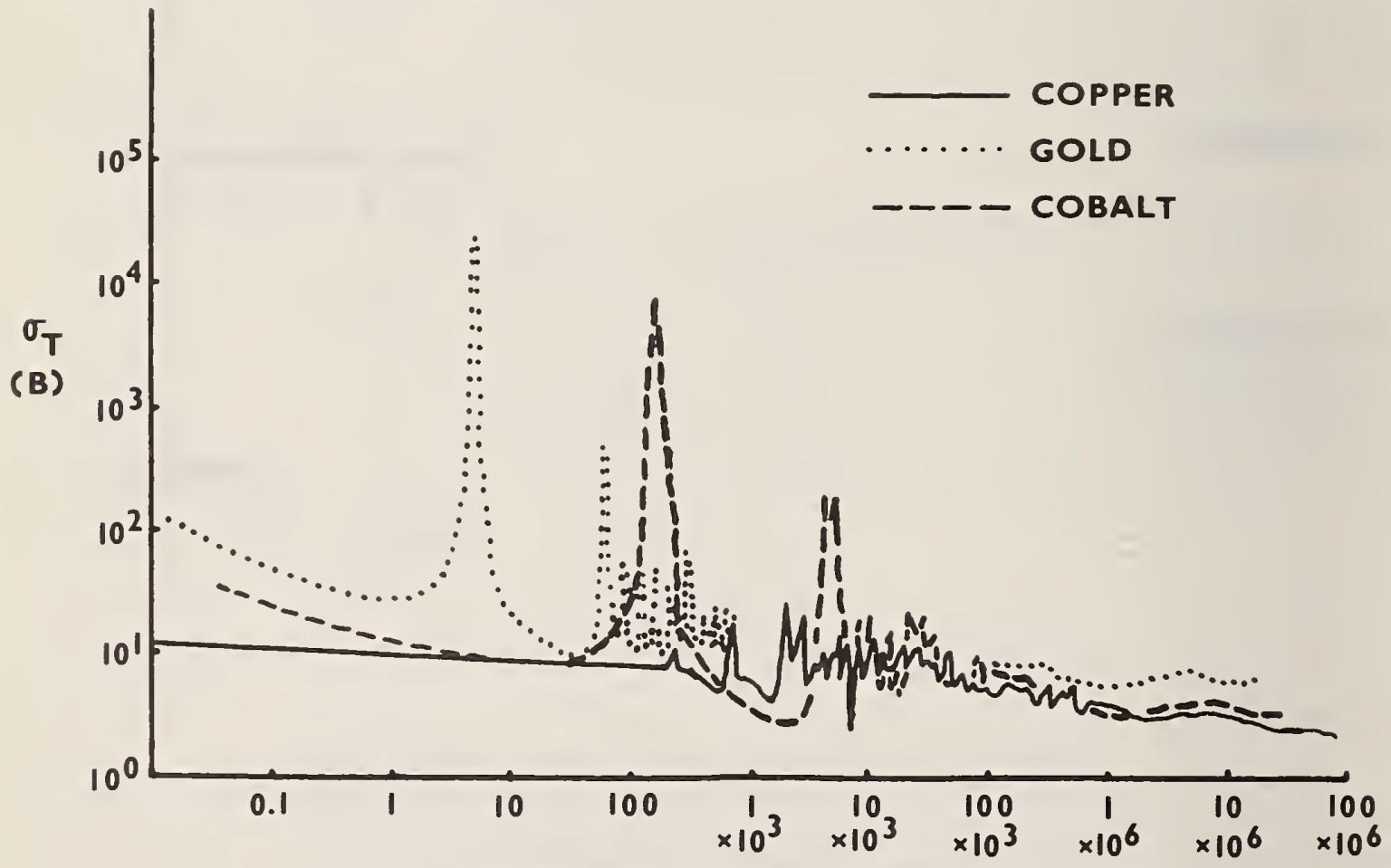

NEUTRON ENERGY (EV)

Figure 2. Neutron cross sections of copper, gold and cobalt as a function of neutron energy. 
since gold is often used in other laboratories. However, since gold has significant neutron self-absorption problems, slight variations in foil thickness can lead to appreciable errors in flux determinations.

The technique used consisted in irradiating a thin foil (0.005 in $\mathrm{Cu}$ foil; 0.001 in $\mathrm{Au}$ foil) of the element of interest in the appropriate facility, then counting the radioactive species formed on a large volume Ge(Li) detector. This detector has been calibrated for absolute photopeak counting efficiency using NBS calibrated radioactive sources. Neutron fluxes were then calculated from nuclear constants. The thermal (sub-cadmium) neutron flux was obtained after correction for epicadmium contribution to the foil activity. For copper, this correction did not exceed $7 \%$, however, for gold this correction was in excess of $25 \%$. No neutron selfshielding corrections were made.

The results of the flux determinations are found in table 2. It is apparent that the thermal neutron flux in tubes RT-1, RT-2 and RT-3 are very similar, with a significantly lower flux in RT-4. The gold foils gave higher flux numbers (relative to those from $\mathrm{Cu}$ measurements) for the tubes RT-1, RT-2 and RT-3, where a larger epicadmium neutron flux exists, but a value very close to that for copper in RT-4, where the neutrons are highly thermalized. Repeated determinations of these flux measurements gave results agreeing well with the original values.

Table 2. Experimental neutron flux-NBSR pneumatic facilities. Sub-Cadmium Neutron Flux-Proximal End $\left(\mathrm{n} . \mathrm{cm}^{-2} \mathrm{Sec}^{-1}\right)$

Detector Foil

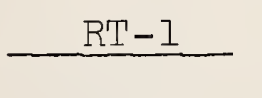

Gold

$7.0 \times 10^{13}$

$6.9 \times 10^{13}$

$\frac{\mathrm{RT}-3}{5.8 \times 10^{13}}$

RT-4

Copper

$$
5.6 \times 10^{13}
$$

$6.3 \times 10^{13}$

$4.9 \times 10^{13}$

$1.4 \times 10^{13}$

$1.3 \times 10^{13}$ 


\section{(3) Neutron Flux Variation Measurements}

In order to obtain accurate and precise results in neutron activation analysis using a nuclear reactor, the geometrical variation of the flux within each irradiation position must be accurately evaluated. Previous studies of the NRL Nuclear Reactor [3] revealed serious neutron flux variations within the sample irradiation area, and thus emphasized the need for such measurements.

The flux measurements for the NBSR were made on the inside dimensions of the polyethylene rabits for the $11 / 2$ in. pneumatic tube system (RT-1 to RT-4). Since the irradiation space available for each facility approximated a right circular cylinder $2.6 \mathrm{~cm}$ in diameter and $8 \mathrm{~cm}$ long, both the circumferential flux distributions and the axial flux distributions were measured. The procedure developed used $3 / 16$ in. disks of copper foil or 20 mil copper wire with gross gamma counting of the copper-64 radioactivity using an automatic sample changer. The copper activity was checked for extraneous radioactivities by counting on a 47cc Ge(Li) detector and 4096 channel PHA and by following the decay characteristics of the foil on the gross gamma counter for over 8 half-lives. Both procedures revealed no detectable radioactivity in either the wire or the foil other than copper-64. All samples from a particular type of measurement were counted under identical conditions.

The results obtained for the circumferential flux distribution of the pneumatic facilities are found in table 3 . In all cases copper foils were used. These results are also shown graphically in figure 3. The foil placement diagram for the circumferential flux measurements is shown in figure 4. It can be readily seen from these results that there is no significant circumferential flux variation at any plane perpendicular to the axis of the right circular cylinder comprising the rabbit irradiation area. 


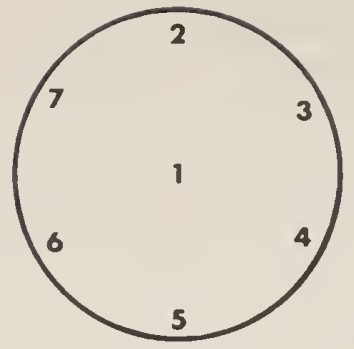

Figure 4. Copper foil placement for circumferential flux measurements in the NBSR pneumatic tubes.

The axial flux measurements were made using the copper wire. A single length of the wire was centered along the rabbit axis and held securely in position with polyethylene foam. After irradiation, the wire was cut into 1 cm sections, weighed, and counted. The results, shown in figures 5 and 6, reveal a serious neutron flux dropoff along the axis of the rabbit. The magnitude of this dropoff over the length of the rabbit ranged from approximately $11 \%$ for $\mathrm{RT}-1$

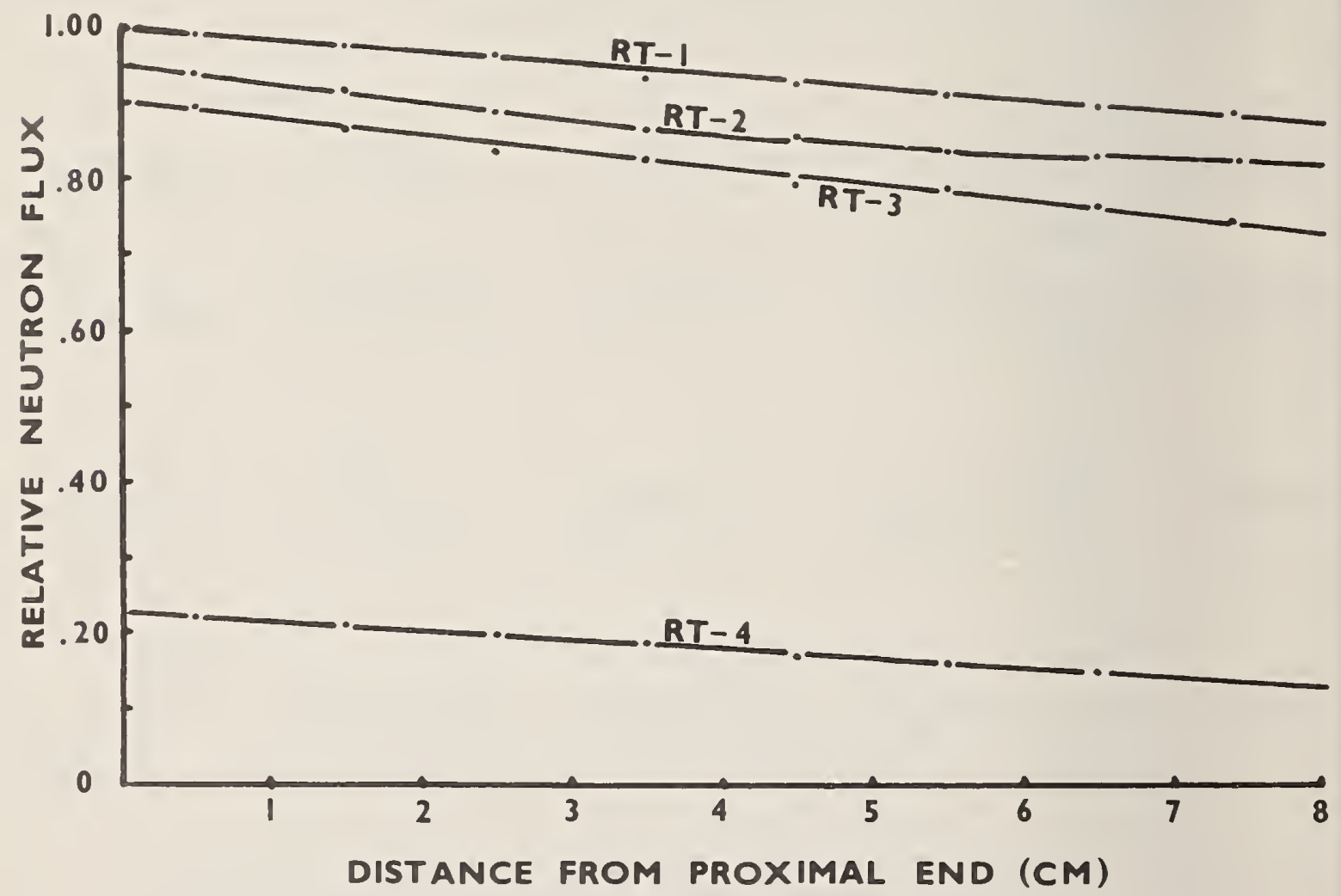

Figure 5. Axial flux distribution in the NBSR pneumatic tubes. 


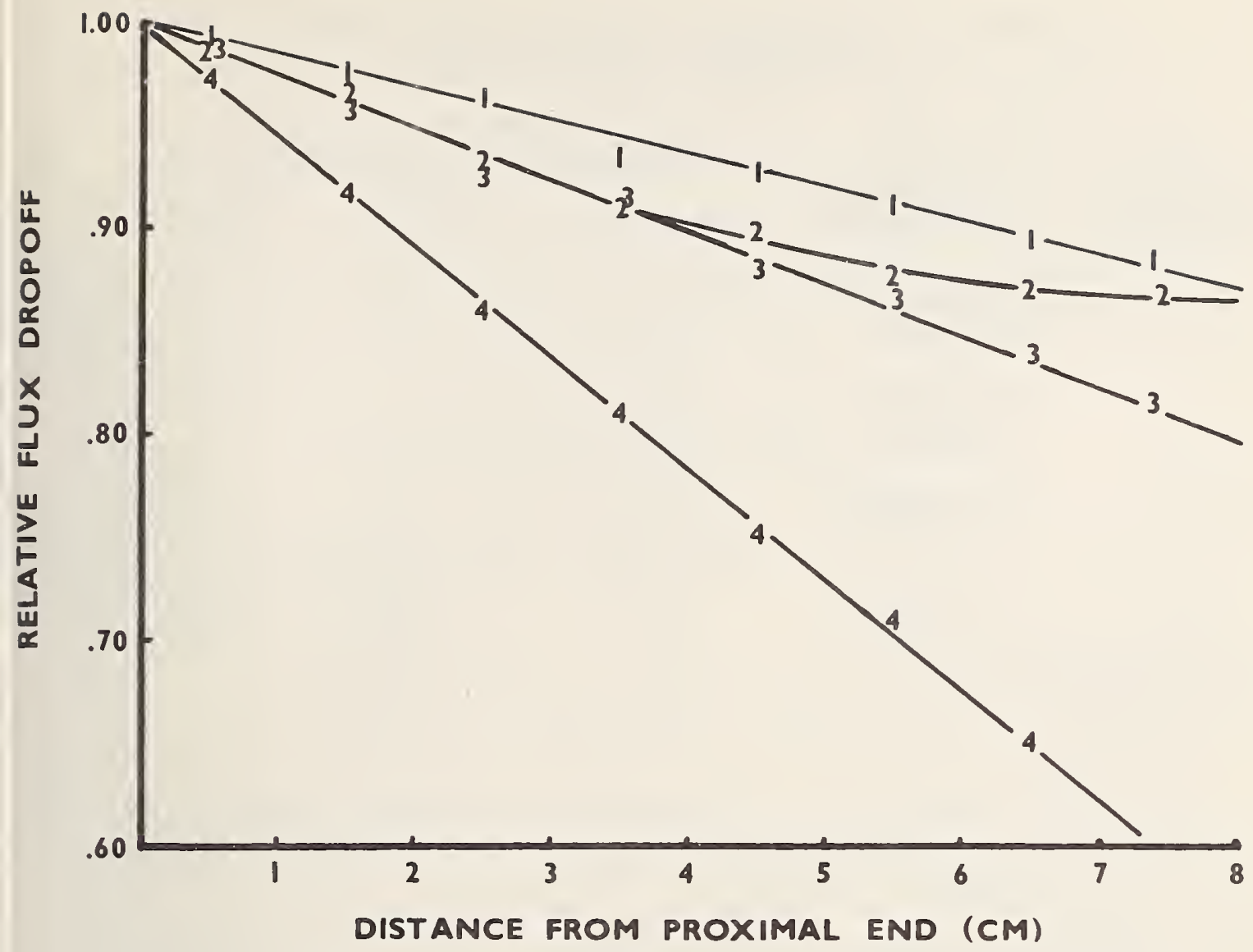

Figure 6. Axial flux dropoff inside the rabbits (normalized to proximal end $\equiv l .00$ for each pneumatic tube).

to over $40 \%$ for RT-4. The flux dropoff was linear in all pneumatic facilities except RT-2.

\section{(4) Neutron Energy Distribution Measurements}

Some preliminary neutron energy distribution measurements have been made, consisting almost entirely of cadmium ratio measurements. These cadmium ratios were made at both ends of all four pneumatic facilities. Three different elemental foils were used, 1 mil thick gold foil, 5 mil copper foil, and $2 \mathrm{mil}$ cobalt foil. Interlocking 20 mil cadmium covers were used for the cadmium shielded measurements.

The results of these measurements are found in table 4. The values for gold and cobalt were obtained primarily 
Pneumatic Location

Facility In Rabbit

RT-1 Proximal

$\mathrm{RT}-1$

$\mathrm{RT}-2$

$\mathrm{RT}-2$

$\mathrm{RT}-3$

$\mathrm{RT}-3$

$\mathrm{RT}-4$

$\mathrm{RT}-4$
Distal

Proximal

Distal

Proximal

Distal

Proximal

Distal
Experimental Cadmium Ratios ${ }^{\mathrm{a}}$
$\underline{\mathrm{Cu}(\mathrm{cd})}$

10

3.7

4.0

$10 \cdot 3$

16.5

87

111
46

64

$\underline{\mathrm{C}} \underline{\mathrm{o}(\mathrm{Cd})}$

29

15.7

15.8

65

80.5

536

582

\section{a} .

For elemental foils using $20 \mathrm{mil}$ cadmium covers.

for intercomparisons with other laboratories, since these two elements are the ones generally used for flux and cadmium ratio measuarements. However, as mentioned previously, the large resonance neutron cross sections (fig. 2) casts some doubt on usefulness of measurements using these foils. The copper foils, which behave as true "infinitely thin" foils throughout the neutron energy range seen in a nuclear reactor, should yield results which can be compared directly to neutron fluxes above and below the cadmium cutoff.

Additional efforts will be expended in the direction of neutron energy measurements in these facilities by the use of threshold detectors. These threshold detectors yield integrated neutron fluxes above their reaction thresholds, and will be used to plot diagrams of the integral neutron flux vs. neutron energy similar to the work done by us at the NRL nuclear reactor [3].

\section{(5) Temperature Measurements}

An extensive series of measurements were made in the pneumatic facilities using stick-on type temperature sensors. 
These sensors were described previously [1] as were the various techniques employed and some preliminary results.

Essentially all of the measurements have been completed at this time, and the results obtained are presented in tables 5, 6, 7 and 8. The equilibrium temperatures were lower than those expected, and therefore maximum irradiation times are based on factors other than temperature, e.g. radiation

Table 5. Radiation damage and temperature measurements at 10 NW in RT-1.

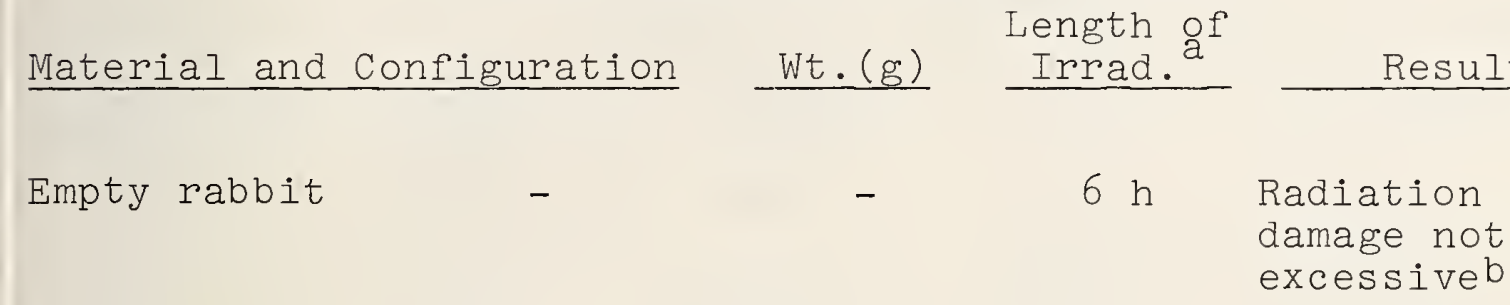

$\begin{array}{llll}\text { Quartz ampoule, empty } & 3.5 \mathrm{~g} & 20 \mathrm{~m} & 170 \pm 10^{\circ} \mathrm{F} \\ \text { Iuead in quartz ampoule } & 0.6 \mathrm{~g} \mathrm{~Pb} & & \\ & 3.5 \mathrm{~g} \text { Quartz } & 20 \mathrm{~m} & 225 \pm 5^{\circ} \mathrm{F} \\ \text { Iron in quartz ampoule } & 0.6 \mathrm{~g} \mathrm{Fe} & & \\ & 3.5 \mathrm{~g} \text { Quartz } & 20 \mathrm{~m} & <180^{\circ} \mathrm{F}\end{array}$

$a_{20}$ minutes required to reach equilibrium temperature

In this and succeeding tables, this phrase means the rabbit is not so brittle as to break upon deceleration in receiver.

Table 6. Radiation damage and temperature measurements at 10 MW in RT-2.

\begin{tabular}{|c|c|c|c|}
\hline Materials and Configuration & Wt. (g) & $\begin{array}{l}\text { igth of } \\
\text { grad. }\end{array}$ & Results \\
\hline Empty rabbit & - & $20 \mathrm{~m}$ & $155 \pm 5^{\circ} \mathrm{F}$ \\
\hline Empty 2 dram poly vial & $3.25 \mathrm{~g} \mathrm{poly}$ & $10 \mathrm{~m}$ & $205 \pm 5^{\circ} \mathrm{F}$ \\
\hline $\begin{array}{l}\text { Empty heat sealed sections } \\
\text { of poly tubing }\end{array}$ & $\begin{array}{l}0.7 \mathrm{~g} \text { poly } \\
(\text { total })\end{array}$ & $10 \mathrm{~m}$ & $205 \pm 5^{\circ} \mathrm{F}$ \\
\hline
\end{tabular}


Table 7. Radiation damage and temperature measurements at $10 \mathrm{NW}$ in $\mathrm{RT}-3$.

\section{Material and Configuration}

Empty rabbit

Empty rabit

Lead pieces (6) in sealed quartz ampoule

Quartz block

Quartz block

Lead and quartz - lead

(single piece) in sealed quartz ampoule

Empty rabbit, containing two 2/5 dram polyvials, with polyvial packing
Length of

$\begin{array}{cr}\text { Wt.(g) } & \frac{\text { Irrad. }}{-} \\ - & 80 \mathrm{~m} \\ & 8 \mathrm{~h}\end{array}$

Results

$$
<110^{\circ} \mathrm{F}
$$

Radiation damage not excessive.

$0.6 \mathrm{~g} \mathrm{~Pb}$

$3.7 \mathrm{~g}$ Quartz

$4 \mathrm{~h}$

$195 \pm 5^{\circ} \mathrm{F}$

$6.0 \mathrm{~g}$

$15 \mathrm{~m}$

$170 \pm 10^{\circ} \mathrm{F}$

$7.0 \mathrm{~g}$

$20 \mathrm{~m}$

$190 \pm 10^{\circ} \mathrm{F}$

$1.0 \mathrm{~g} \mathrm{~Pb}$

$3.2 \mathrm{~g}$ Quartz $10 \mathrm{~m}$ $>220^{\circ} \mathrm{F}$

$8 \mathrm{~h}$

Radiation damage not excessive to rabbit or polyvials. Poly foam crumb ly .

Table 8. Radiation damage and temperature measurements at 10 MW in RT-4.

Material and Configuration Wt.(g) $\begin{gathered}\text { Length of } \\ \text { Irrad. }\end{gathered}$ Empty rabbit
Empty rabit

Lead in sealed quartz ampoule

$-16 \mathrm{~h}$

Results

Radiation damage not excessive

\begin{tabular}{|c|c|c|}
\hline - & $16 \mathrm{~m}$ & $105 \pm 5^{\circ} \mathrm{F}$ \\
\hline $0.6 \mathrm{~g} \mathrm{~Pb}$ & $15 \mathrm{~m}$ & $<130^{\circ} \mathrm{F}$ \\
\hline
\end{tabular}

damage to the sample carrier. Irradiations of up to 8 hours are made routinely in the polyethylene rabbits at a thermal neutron flux of $5 \times 10^{13} \mathrm{n} \cdot \mathrm{cm}^{-2} \mathrm{sec}^{-1}$. 
Initially, reactor operations would permit temperatures of up to $200^{\circ} \mathrm{F}$ in the rabbit facilities. However, a few tests were made of the melting point of these rabbits and the effect of irradiation on the melting point. The results of these tests are given in table 9. As a result of these tests, the maximum permissable temperature in the pneumatic facilities was raised to $240^{\circ} \mathrm{F}$.

Table 9. Heat resistance of polyethylene rabbits: effect of irradiation timea.

Length of

Irradiation

none

\begin{tabular}{|c|c|c|c|c|}
\hline rradiation & $230^{\circ} \mathrm{F}$ & $240^{\circ} \mathrm{F}$ & $260^{\circ} \mathrm{F}$ & $280^{\circ} \mathrm{F}$ \\
\hline none & none & none & $\begin{array}{l}\text { slight } \\
\text { softening }\end{array}$ & $\begin{array}{l}\text { completely } \\
\text { melted }\end{array}$ \\
\hline $10 \mathrm{sec}$ & none & none & $\begin{array}{l}\text { slight } \\
\text { softening }\end{array}$ & $\begin{array}{l}\text { mostly } \\
\text { melted }\end{array}$ \\
\hline $1 \min$ & none & none & $\begin{array}{l}\text { slight } \\
\text { softening }\end{array}$ & $\begin{array}{l}\text { completely } \\
\text { deformed }\end{array}$ \\
\hline $10 \mathrm{~min}$ & none & none & $\begin{array}{l}\text { slight } \\
\text { softening }\end{array}$ & $\begin{array}{l}\text { transparent } \\
\text { and starting } \\
\text { to deform }\end{array}$ \\
\hline
\end{tabular}

Melting point of conventional polyethylene is $2250^{\circ} \mathrm{F}$; high density polyethylene $\sim 270^{\circ} \mathrm{F}$.

Rabbits irradiated in RT-I.

\section{(6) Pressure Measurements}

When the request was made for the irradiation of freeze dried biological materials in the NBSR, a number of questions were raised which could not be definitively answered. One of these questions involved the danger of explosion of a quartz ampoule due to the pressure generated by gaseous decomposition products of the material under irradiation. To effectively answer this question, and to establish criteria for the safe irradiation of a variety of aqueous solutions, 
organic materials, and biological materials, a series of pressure measurements was initiated. The pressure testing system used to test irradiated samples is shown in figure 7 . It consists of an appropriate sized rubber balloon, a section of polyethylene tubing which will fit inside the balloon but is still large enough to hold the irradiated quartz ampoule, and a gas-tight clamp for the open end of the balloon. To make a pressure measurement, an irradiated sample (inside the quartz ampoule) is inserted into the tubing and the balloon, the end of the balloon clamped shut and the ampoule struck smartly with a hammer. The polyethylene tubing prevents the shattered quartz from puncturing the balloon, and the pressure inside the ampoule estimated from the degree of expansion of the balloon.

Tests with a hypodermic syringe (less needle) snugly fitted to the balloon opening indicated that $1 / 2 \mathrm{~cm}^{3}$ of gas could be readily detected. The quartz ampoules being tested had volumes of $1 \mathrm{~cm}^{3}$ or greater, therefore excess pressures of 1 atm could most certainly be measured. Since even poor quality glass blowing produces ampoules which can readily withstand 2 atm excess pressure, and good quality quartz glass blowing can produce ampoules which will withstand internal pressures in excess of 5 atm excess pressure, a preliminary limit for testing purposes of $11 / 2$ atm excess pressure was established.

\section{RUBBER BALLOON}

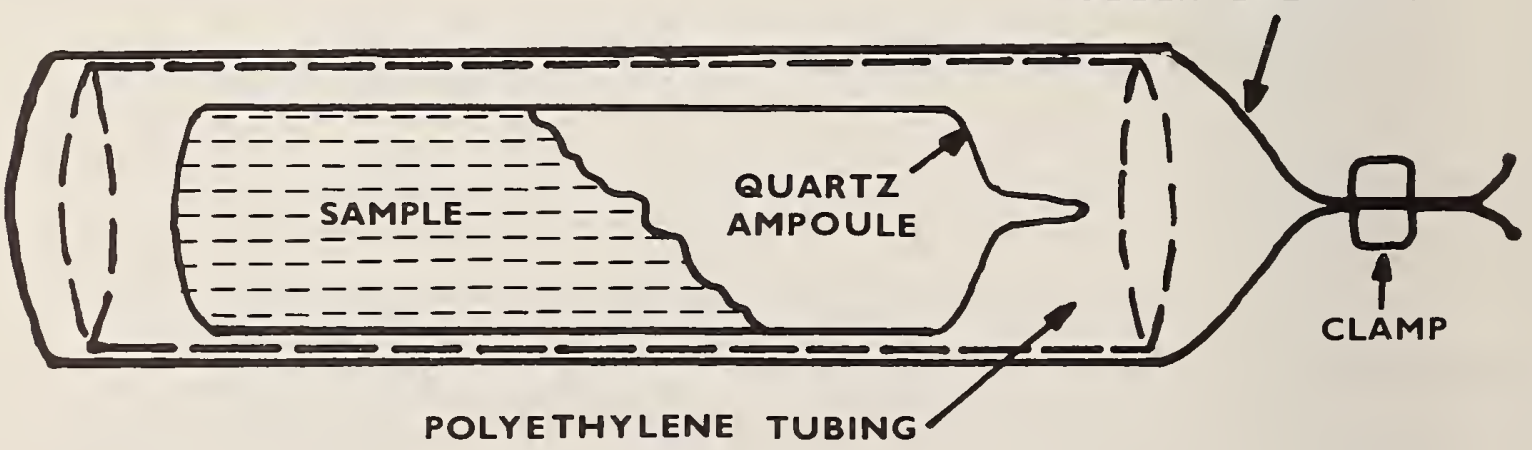

Figure 7. Device used to measure pressures generated from irradiated samples. 
The results of these pressure tests are found in table 10. From these results it can be seen that freeze-dried biological materials produce only small quantities of gaseous products upon irradiation in the pneumatic facilities RT-I, RT-3 and RT-4. For example, $1 / 2$ gram of freeze-dried orchard leaves, irradiated for 6 hours in RT-3 at a neutron flux of $5 \times 10^{13} \mathrm{n} . \mathrm{cm}^{-2} \mathrm{sec}^{-1}$ produced only about $1 \mathrm{~cm}^{3}$ of gaseous decomposition products (which produced approximately 1 atm of excess pressure in the ampoule size used). These conditions of excess pressure are well within the tentative limits set up for these tests.

Table 10. Pressure measurements on neutron irradiated samples.

Length

Sample Wt (g) of Irrad. Facility Results $^{\mathrm{a}}$

Orchard leaves

$0.25 \quad 2 \mathrm{~h} \quad \mathrm{RT}-1$ freeze dried

\section{Alfalfa, freeze} dried

$0.252 \mathrm{~h}$

$\mathrm{RT}-3$ pressure buildup

Blood, freeze

0.25

$2 \mathrm{~h}$

$\mathrm{RT}-3$

No significant dried

$0.252 \mathrm{~h}$

$\mathrm{RT}-3$ pressure buildup

Liver, freeze dried

Water, in heat- 2.0

$5 \mathrm{~m}$

$\mathrm{RT}-3$

No significant buildup

sealed polyethylene

Orchard leaves

0.50

$8 \mathrm{~h}$

$\mathrm{RT}-4$

No significant pressure buildup

freeze dried

Orchard Leaves, freeze dried

$0.50 \quad 6 h \quad R T-3$

Small pressure buildup

a Limit of detection $\sim 1 / 2 \mathrm{~cm}^{3}$; "small pressure buildup" estimated to be $1 / 2-1 \mathrm{~cm}^{3}$. 
b. Long Term Irradiation Facility (LTIF)

The flux measurements made on the LTIF Vertical Thimble (see [4] for details of construction) included only neutron flux measurements and cadmium ratio determinations. Since this facility has its own recirculating $\mathrm{D}_{2} \mathrm{O}$ cooling system, the temperature measurements were not immediately essential. The measurement of pressures due to sample decomposition have not yet been made on this facility, at least partly due to the probable applicability of those measurements made with the pneumatic tubes.

The flux variation measurements on this facility were made using two different techniques. For the vertical flux measurements a copper wire inside of sealed polyethylene tubing was quickly inserted the entire length of the vertical irradiation facility and irradiated for $30 \mathrm{~m}$ at a power level of $10 \mathrm{KW}$. At the end of the irradiation time, the copper wire was quickly removed from the reactor, the polyethylene tubing removed, and the copper wire cut into small pieces for gross $\gamma$ counting of the 12.9 h copper-64 radioactivity. At the lower end, where the activity level was relatively high, the wire was cut into $1 / 2$ inch lengths. As the induced radioactivity decreased in the upper portions of the wire (above the core area), sections were increased to 1 inch, and finally to 6 inches at a point considerably above the upper core area. After this vertical flux diagram was obtained, the piece having the highest specific activity (from the core gap area) was taken for an absolute flux determination.

Circumferential flux measurements were made during irradiations for the cadmium ratios. These measurements were taken using suitably weighted polyethylene rabits at the end of a nylon fishing line. The copper wire was wrapped around the rabbit in which the bare copper foil was irradiated. The cadmium covered copper foil had its separate irradiation. This set of two irradiations was necessary for each area measured. Four areas were measured this way, as shown in 
figure 8 and table 11 .

The results of these measurements are shown in figure

8 and tables 11 and 12. Figure 8 shows the vertical

distribution of the total neutron flux in the LTIF, and demonstrates the effectiveness of the core gap area as a region of maximum thermal neutron flux. The maximum neutron

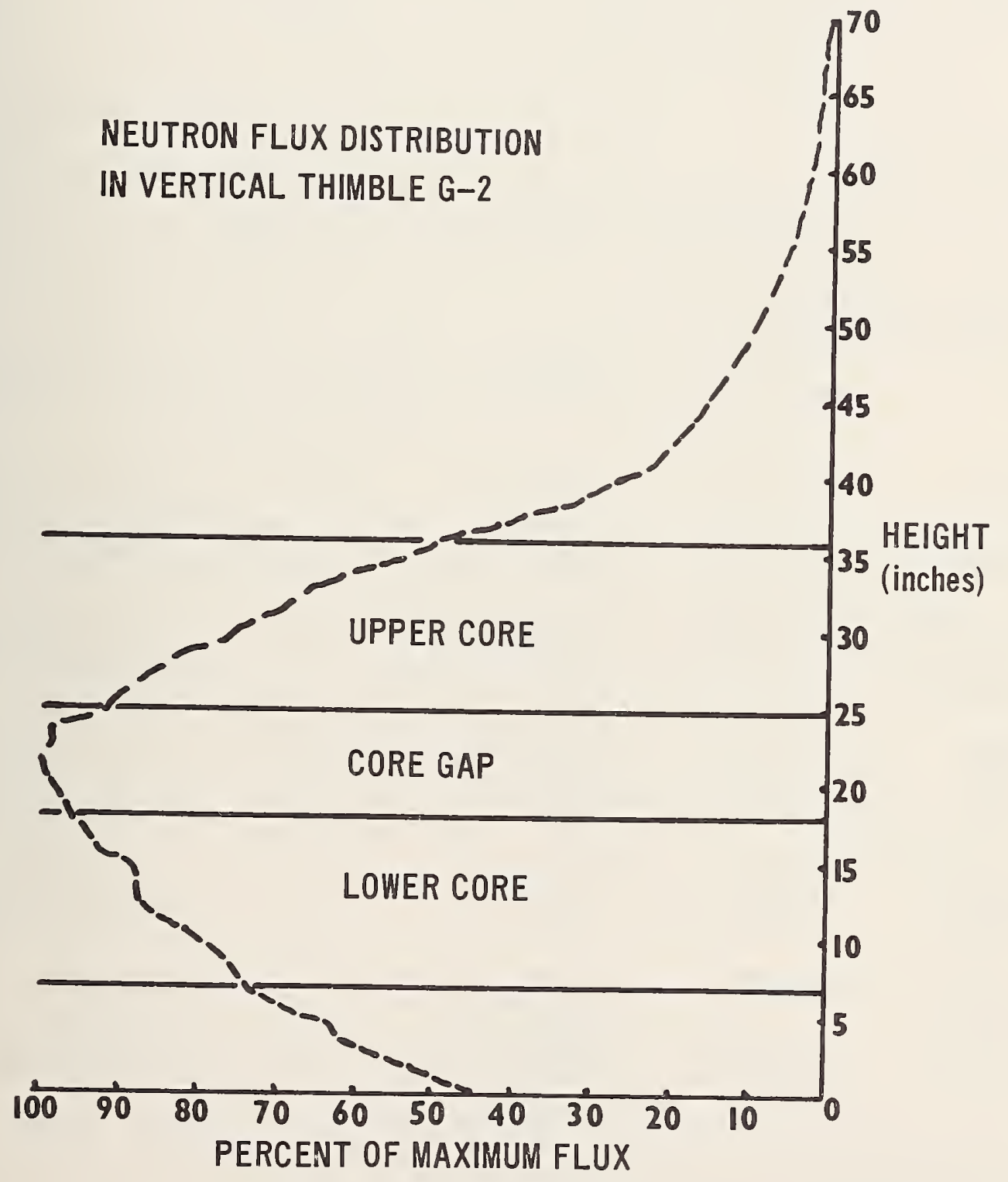

Figure 8. Vertical distribution of the total neutron flux in the NBSR LTIF. 
Table 1l. Cadmium ratios in the LTIF.

\section{Area}

Below core

Lower core

Core gap

Above core
Height (in.)

0.5

11

19.5

56
Cadmium Ratio

(Copper)

84.1

32.8

54.9

1830

Table 12. Circumferential flux variation in the NBSR LTIF irradiation facility.

Location in LTIF

\begin{tabular}{|c|c|c|c|c|}
\hline Area & Height (in) & $\begin{array}{c}\text { Location } \\
\text { Rabbit } \\
\end{array}$ & $\begin{array}{r}\text { in No. of } \\
\text { Monitors } \\
\end{array}$ & $\begin{array}{c}\text { Relative } \\
\text { Neutron Flux }\end{array}$ \\
\hline $\begin{array}{l}\text { On bottom } \\
\text { On bottom } \\
\text { On bottom }\end{array}$ & $\begin{array}{l}0.5 \\
1.8 \\
3.0\end{array}$ & $\begin{array}{l}\text { Bottom } \\
\text { Middle } \\
\text { Top }\end{array}$ & $\begin{array}{l}9 \\
9 \\
9\end{array}$ & $\begin{array}{l}1.000 \pm 0.010^{c} \\
1.122 \pm 0.008 \\
1.238 \pm 0.009\end{array}$ \\
\hline $\begin{array}{l}\text { Lower core } \\
\text { Lower core } \\
\text { Lower core }\end{array}$ & $\begin{array}{l}11.5 \\
12.8 \\
14.5\end{array}$ & $\begin{array}{l}\text { Bottom } \\
\text { Middle } \\
\text { Top }\end{array}$ & $\begin{array}{l}9 \\
9 \\
9\end{array}$ & $\begin{array}{l}1.000 \pm 0.018 \\
1.055 \pm 0.026 \\
1.088 \pm 0.015\end{array}$ \\
\hline $\begin{array}{l}\text { Core gap } \\
\text { Core gap } \\
\text { Core gap }\end{array}$ & $\begin{array}{l}20 \cdot 0 \\
21 \cdot 3 \\
22 \cdot 5\end{array}$ & $\begin{array}{l}\text { Bottom } \\
\text { Middle } \\
\text { Top }\end{array}$ & $\begin{array}{l}9 \\
9 \\
9\end{array}$ & $\begin{array}{l}1.000 \pm 0.021 \\
0.997 \pm 0.016 \\
0.980 \pm 0.018\end{array}$ \\
\hline $\begin{array}{l}\text { Upper core } \\
\text { Upper core } \\
\text { Upper core }\end{array}$ & $\begin{array}{l}28.5 \\
29.8 \\
31.0\end{array}$ & $\begin{array}{l}\text { Bottom } \\
\text { Middle } \\
\text { Top }\end{array}$ & $\begin{array}{l}9 \\
9 \\
9\end{array}$ & $\begin{array}{l}1.000 \pm 0.081 \\
0.837 \pm 0.170 \\
0.725 \pm 0.092\end{array}$ \\
\hline $\begin{array}{l}\text { Above core } \\
\text { Above core } \\
\text { Above core }\end{array}$ & $\begin{array}{l}56.5 \\
57.8 \\
59.0\end{array}$ & $\begin{array}{l}\text { Bottom } \\
\text { Middle } \\
\text { Top }\end{array}$ & $\begin{array}{l}9 \\
9 \\
9\end{array}$ & $\begin{array}{l}1.000 \pm 0.011 \\
0.885 \pm 0.013 \\
0.774 \pm 0.011\end{array}$ \\
\hline
\end{tabular}

\footnotetext{
a Measured from bottom of polyethylene rabbit (see figure 8). Normalized to bottom $\equiv 1.00$.

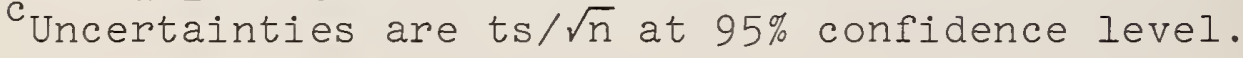


flux for 10 MW operation was found to be approximately $1.1 \times 10^{14} \mathrm{n} . \mathrm{cm}^{-2} \mathrm{sec}^{-1}$. The cadmium ratios are given in table 11. The core gap cadmium ratio of 55 is significantly higher than the core area, which has a cadmium ratio of approximately 33. All of these cadmium ratios are somewhat higher than would normally be expected, due to the highly thermalizing effect of the $\mathrm{D}_{2} \mathrm{O}$ filled thimble. The copper cadmium ratio of 1830 at a hight of 56 inches in the thimble (with a 10 MW neutron flux of $\sim 5 \times 10^{12} \mathrm{n} . \mathrm{cm}^{-2} \mathrm{sec}^{-1}$ ) suggests the potential use of this facility for obtaining a highly thermalized neutron environment. The results of the circumferential flux distributions in the LTIF reveal a small but significant variation in the neutron flux from side-to-side in the thimble. This variation is strongly dependent upon the area chosen for the irradiation, but usually is less than $10 \%$ over the outer diameter of the polyethylene rabits used for the measurements.

\section{c. Conclusions}

The NBSR has been characterized for many of its irradiation parameters, both for the pneumatic facilities RT-1, RT-2, RT-3 and RT-4, and for the LTIF (G-2 Vertical Thimble). While some additional measurements remain to be made, the irradiation facilities available for use are revealed as a set of extremely useful and convenient facilities under which a wide variety of irradiation environments can be obtained.

(D. A. Becker and P. D. LaFleur)

2. Installation and Operation of a Compton-Suppressed Gamma Scintillation Spectrometer

The Compton-suppressed gamma-gamma coincidence scintillation spectrometer, with its associated equipment and lead shielding was obtained from the Naval Radiological Defense Laboratory [5] and has been installed and made operational. (fig. 9). The individual modular equipment has been checked for damage during shipment and was found to be in proper 


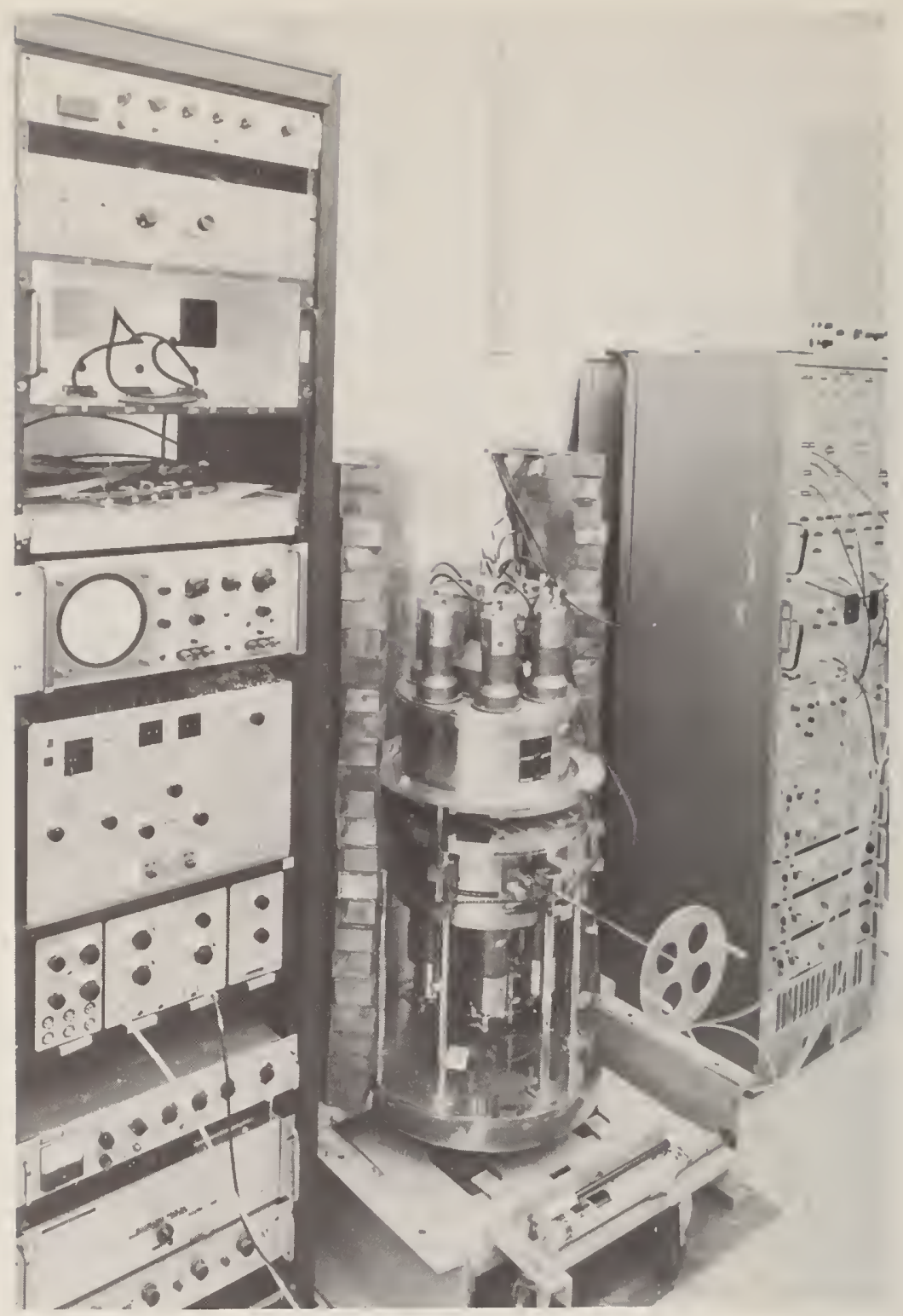

Figure 9. Partially shielded Compton-suppressed gammaray scintillation spectrometer with associated electronics.

order. The system has been interconnected and successfully operated in the gamma sum, and the gamma-gamma sum coincidence modes.

a. Modes of Operation

(I) Gamma Sum Anticompton

In this mode, the signal pulses from each of the two central crystals are linearly summed into a preamplifier, 
then fed to an amplifier. Two outputs are taken from the amplifier, a delay output directly fed to a 400 channel analyzer as signal, the other, a prompt output in coincidence with the annulus signal gated to block the signal pulse any time the sum crystal pulse is in coincidence with the annulus The block diagram of this mode of operation is shown in figure $10 a$.
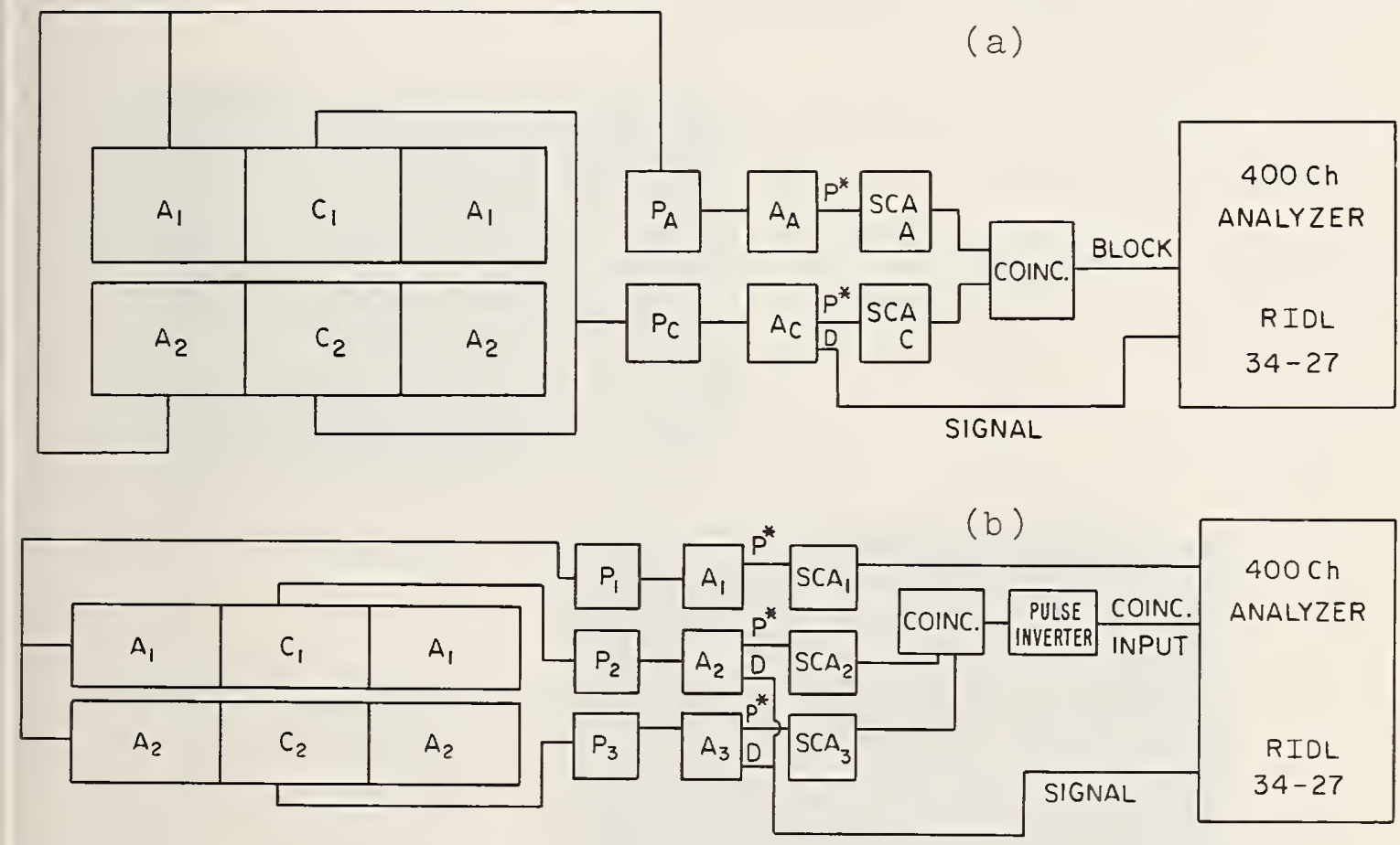

$C_{1}$ - CRYSTAL I

$\mathrm{C}_{2}$ - CRYSTAL 2

$A_{1}$-ANNULUSI

$A_{2}-A N N U L U S 2$

$P^{*}$-PROMPTOUTPUT

$D$ - DELAYED OUTPUT
$P_{1}$ - PREAMPLIFIER FOR ANNUL

$P_{2}$ - PREAMPLIFIER FOR CRYSTALI

$\mathrm{P}_{3}$ - PREAMPLIFIER FOR CRYSTAL 2

$A_{1}$ - AMPLIFIER FOR ANNUL

$A_{2}-A M P L I F I E R$ FOR CRYSTAL I

$A_{3}-A M P L I F I E R$ FOR CRYSTAL 2

SCA-SINGLE CHANNEL ANALYZER

COINC.-COINCIDENCE UNIT

Figure 10. Schematic of the Compton-suppressed gamma-gamma scintillation spectrometer. (a) Block diagram of the coincidence system used in the gamma sum mode with compton supression. (b) Block diagram of the coincidence system used in the gamma-gamma coincidence sum mode with Compton suppression. 
(2) Gamma-Gamma Sum Coincidence

In this mode, only the pulses from the two central crystals which are in coincidence are accepted. These pulses are linearly summed after the amplifiers and fed as a signal to a 400 channel analyzer. All pulses from the annulus in coincidence with the signal pulses were fed to a blocking gate to provide Compton suppression. The block diagram of this mode of operation is shown in figure lob.

b. Results

(1) Gamma Sum Anticompton

The system was used to count ${ }^{60}$ Co in the gamma sum and the gamma sum Compton-suppressed modes. The Compton suppression reduced the compton continuum by about a factor of 4 while reducing the background a factor of about 2. A Comparison of spectra of ${ }^{60} \mathrm{Co}$ with and without Compton-suppression is shown in figure 11 .

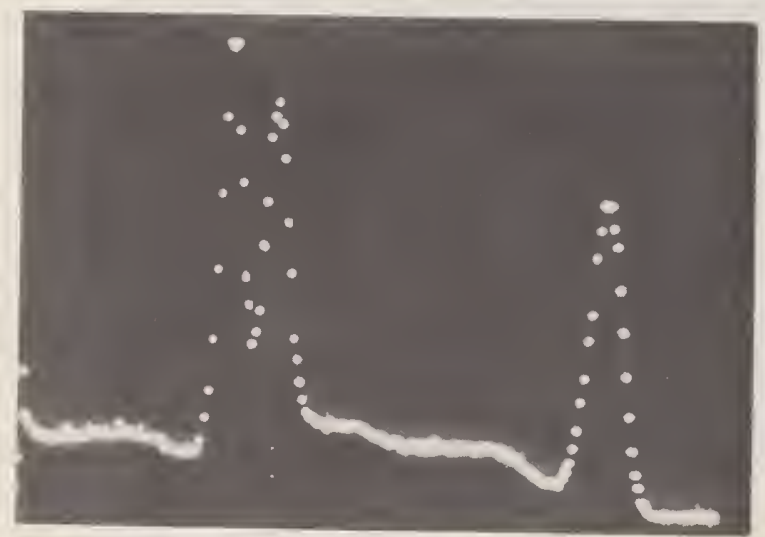
A. GAMMA SUM NO COMPTON SUPPRESSION

B. GAMMA SUM WITH COMPTON SUPPRESSION

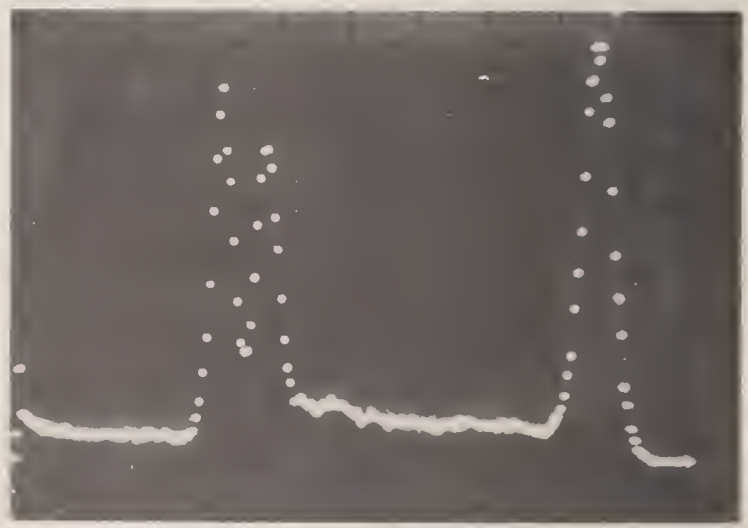

Figure 11. Gamma sum spectra of cobalt-60. 
(2) Gamma-Gamma Sum Coincidence

The system was used to count ${ }^{60} \mathrm{Co}$ and ${ }^{137} \mathrm{Cs}$ in the gammagamma coincidence mode with and without compton suppression. Non-coincidence events were reduced by a factor of between 15 and 20. The best results were obtained using gamma-gamma coincidence with Compton suppression as can be seen in figures 12 and 13.

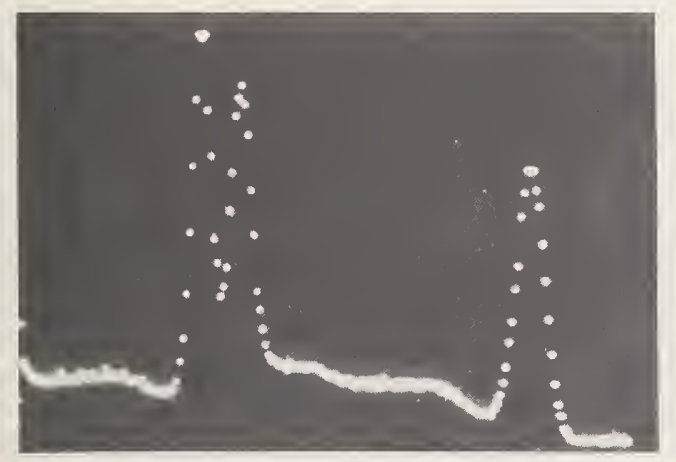
A. GAMMA SUM WITHOUT COMPTON SUPPRESSION

B. GAMMA-GAMMA SUM COINCIDENCE WITHOUT COMPTON SUPPRESSION
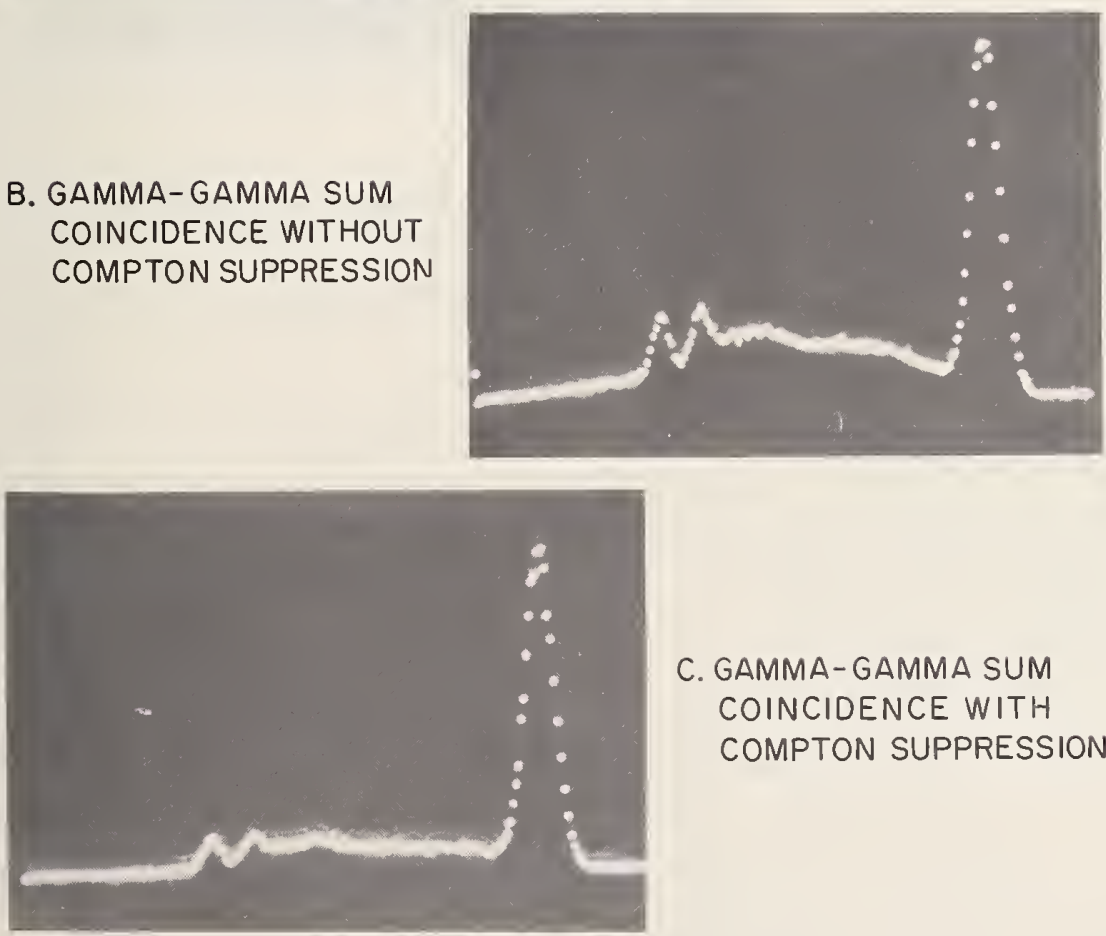

C. GAMMA-GAMMA SUM COINCIDENCE WITH COMPTON SUPPRESSION

Figure 12. Gamma-gamma coincidence spectra of cobalt-60. 


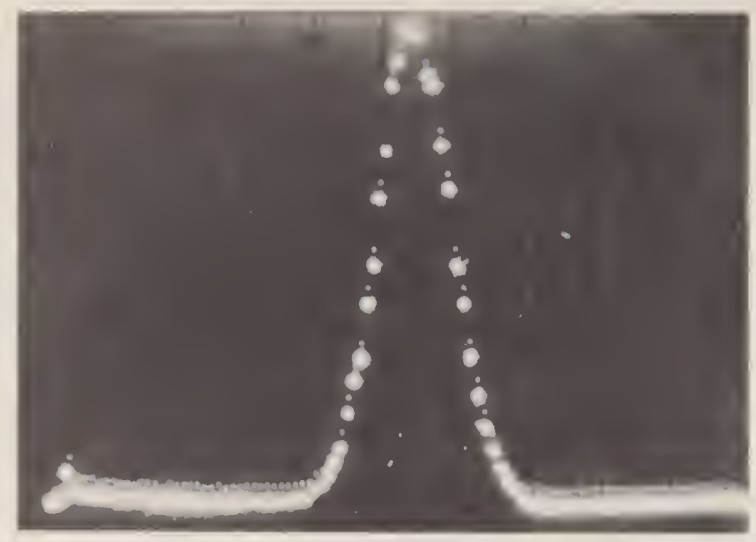

A. GAMMA SUM

B. GAMMA-GAMMA SUM COINCIDENCE

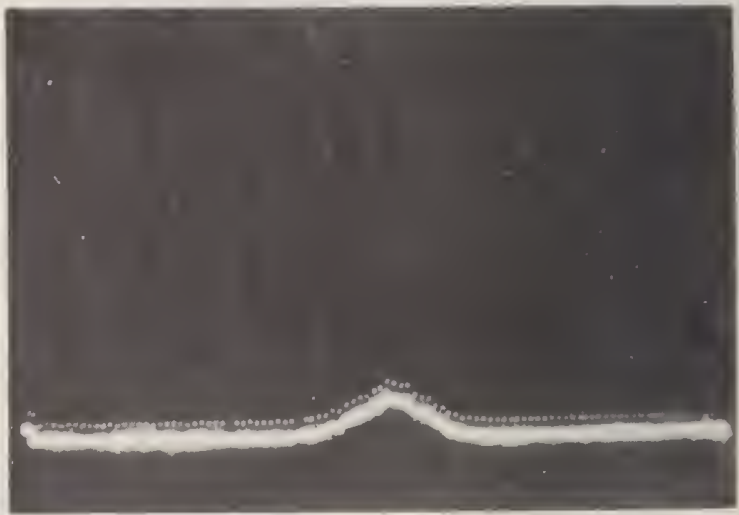

Figure 13. Gamma-gamma coincidence spectra of cesium-137. (H. L. Rook and D. A. Becker)

C. Research Activities

1. Characterization of Medieval Glass by Neutron Activation Analysis

a. Introduction

resedrcn carried out by J. S. Olin of the Smithsonian Institution and E. V. Sayre of Brookhaven National Laboratory [6] and by I. Perlman and F. Asaro of the Lawrence Radiation Laboratory [7] has shown that neutron activation analysis can be a valuable aid in determining the provenance of pottery samples. The Smithsonian was interested in learning whether similar methods could be applied to the characterization of medieval glass to help decide questions of the 
authenticity of stained glass windows, which panes are original and which replacements, and possibly even the precise origin of a partciular window. To determine the feasibility of this approach, a joint study was begun using the NBS reactor and personnel from both the Smithsonian and NBS, as reported previously $[8]$.

b. Experimental Procedure

The first experiments were carried out to gain information about the composition and homogeneity of medieval glass and also to find out whether reliable data could be obtained from very small samples (10-100 mg) since this is probably close to the maximum which could be expected to be made available from an actual window. The general approach followed was similar to that used by Coleman and Wood [9] in their work with plate glass fragments. First, a small sample ( $10 \mathrm{mg}$ ) was irradiated for 2 seconds at $6 \times 10^{13} \mathrm{n} \cdot \mathrm{cm}^{-2} \cdot \mathrm{sec}^{-1}$ and used to obtain information on major constituents such as $\mathrm{Na}, \mathrm{K}$, $\mathrm{Ca}, \mathrm{Al}, \mathrm{Cu}$, and $\mathrm{Sb}$. Vanadium and indium were also observed if present in these samples. A larger sample (50-100 mg) was then irradiated for $15 \mathrm{~min}-1$ hr to obtain information about minor and trace constituents. Initial plans were to dissolve these large samples and to remove ${ }^{24} \mathrm{Na}$ with HAP and $122,124 \mathrm{Sb}$ by extraction of the chloride complex with isopropyl ether. However, because of the high levels of $\mathrm{Cu}$ and $\mathrm{K}$ present in many of the samples, the value of the HAP separation was reduced and therefore this step was eliminated and the only chemistry used was separation of Sb from samples containing high concentrations of this element. A 47-cc Ge(Li) detector was used for analysis of all samples.

To check out the reliability of the procedure, analyses were carried out on samples of simulated ancient glasses which had been prepared by Corning and distributed by Dr. Robert Brill to various interested laboratories, including the Smithsonian, for the purpose of checking different analytical methods. The 
results obtained with these glasses are shown in table 13 and it is clear that even in these very preliminary analyses, the reproducibility is quite satisfactory.

Because of the high manganese levels in samples "A", "B", and " $D$ ", the analyzer dead time during the measurement of $A l$ and $V$ activities was often as high as 30-40\%. Although no gain shift or loss of resolution could be observed, the high dead time created a problem in the choice of suitable standards for these elements. The problem was solved by using NBS Standard Reference Material (SRM) steels, which often have high Mn concentrations, as standards. SRM 344, which has $0.57 \% \mathrm{Mn}$ and $1.16 \% \mathrm{Al}$, was used for an Al standard, and SRM $30 \mathrm{f}$ with $0.79 \% \mathrm{MN}$ and $0.18 \% \mathrm{~V}$ for a $\mathrm{V}$ standard. Using samples of these, it was fairly easy to get a standard with about the same ${ }^{56}$ Mn activity and thus about the same dead time as the sample. This eliminated the need for decay corrections on these short-lived activities. SRM lole with $1.77 \%$ Min was used as a Mn standard.

The results shown in Table 13 were all obtained on glasses which had been prepared under modern conditions and should have had good homogeneity. The samples to be used for medieval glass analysis had been obtained by Mrs. Olin from the The Cloisters, Metropolitan Meseum of Art, through the courtesy of Miss Jane Hayward and included two sets of fragments. One set was blue glass, designated as probably l3th-l4th century, and the other was a greenish "bullseye" glass designated "Paris - 1775." Although this second set could not really be considered medieval, these two sets of fragments were used to determine the degree of homogeneity to be expected from glasses which had not been prepared under modern conditions. Some of the results obtained are shown in table 14 and it can be seen that in cases where there are plenty of counts and no interferences, e.g. Na in both glasses and Co and La in the blue glass, good precision 
Table 13. Results of activation analysis of simulated ancient glasses, \%.

Element

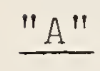

"B"

"C"

"D"

$\mathrm{Na}$

12.34

14.09

12.46

14.56

12.82

11.64

13.93

$13 \cdot 36$

0.990

0.951

0.853

0.891

$\mathrm{Cu}$

1.11

1.08

2.27

2. 34

1.04

2.24

1.05

2.22

1.07

0.312

1.01

0.310

0.92

0.95

Mn

0.816

0.190

0.0007

0.280

0.793

0.179

0.787

0.183

0.0011

0.417

0.474

$\mathrm{Sb}$

$$
\begin{aligned}
& 1.50 \\
& 1.24 \\
& 1.26 \\
& 1.12
\end{aligned}
$$

0.31

0.32

0.30

0.34

$\mathrm{Ba}$

8.71

8.85

7.68

8.08

Co

0.11

0.030

0.118

0.034

0.124

0.87

0.66

0.67

K

$\begin{array}{rr}2.30 & 9.74 \\ -\quad 2.07 & 9.62 \\ 2.39 & \end{array}$

V

0.0046
0.0042

0.017

0.0395

0.0088

0.019

0.018

0.0093

0.015

AI

0.47
0.43
0.44

2.21
2.19
2.22

0.422

0.419

0.419

0.014

0.0394

2.56

2.54

can be obtained. Where there are few counts or interfering peaks, e.g. $\mathrm{K}$ and $\mathrm{Fe}$, the precision is much poorer. On the basis of these results it was concluded that differences of less than $\pm 10 \%$ could not be regarded as significant for most elements. 
Table 14. Homogeneity of Medieval and 18th Century glasses.

\section{Concentrations, \%}

\section{Blue Glass, XIII-XIV}

$\underline{\text { Element }}$

$\begin{array}{lll}\mathrm{Na} & 0.96 \quad \pm 0.04^{\mathrm{a}} \\ \mathrm{K} & 18.9 \quad \pm 2.7^{\mathrm{a}} \\ \mathrm{La} & 0.00404 \pm 0.00008^{\mathrm{b}} \\ \mathrm{Co} & 0.114 \pm 0.001^{\mathrm{b}} \\ \mathrm{Fe} & 0.93 & \pm 0.25^{\mathrm{b}} \\ \mathrm{Cu} & 0.38 & \pm 0.03^{\mathrm{a}} \\ \mathrm{Sb} & 1.08 & \\ \mathrm{Al} & 0.82 & \end{array}$

Clear "Bullseye" Glass Paris, 1775
$4.08 \pm 0.05^{c}$
$3.51 \pm 0.62^{c}$
$0.0015 \pm 0.0001^{\mathrm{c}}$
$0.00066 \pm 0.00006^{c}$
$0.57 \pm 0.06^{c}$
0.404
1.66

Sta. deviation of the mean; $n=4$ $\mathrm{c}_{n-8}^{n-5}$

Also observed in the blue glass: In, Ca, Sc, Zn, Rb. Also observed in the "bullseye" glass: Ca, Sc, Zn, Sm.

The data in table 14 also represents the results of a study of various cleaning methods and it was concluded that the specific type of cleaning is relatively unimportant.

c. Results on Medieval Glass Samples

In addition to the two sets of fragments, five glass samples, all designated as being from Bourges, XII or XIII century, were analyzed using the procedure described above. Figure 14 shows how these glasses were sampled. This is a typical piece, about 2 in. long, with a cut-out portion along one edge where samples for analysis were removed. Samples from this glass were used for one of the homogeneity experiments and so more sample was removed than would be the case for an ordinary analysis. The results obtained on these five samples are listed in table 15. 


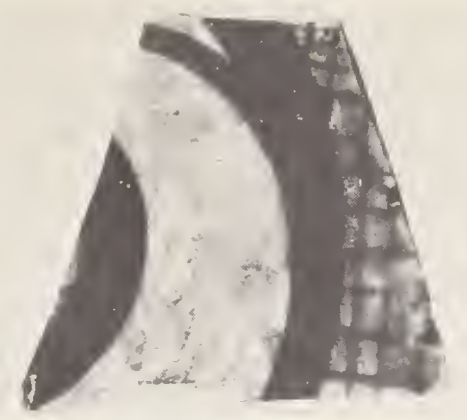

Figure 14. Fragment of medieval glass with sample cut from one edge. (Photograph courtesy of the Smithsonian Institution.)

Table 15. Results of analysis of Bourges glasses, \%

\begin{tabular}{|c|c|c|c|c|c|}
\hline Element $^{a}$ & \multicolumn{4}{|c|}{ Clear Glass } & Blue Glass \\
\hline & No. 1 & No. 2 & No. 3 & No. 4 & No. 5 \\
\hline $\mathrm{Na}$ & 9.8 & 1.45 & 4.2 & 0.45 & 14.5 \\
\hline K & $<2$ & 13.8 & 3.8 & 10.0 & $<2$ \\
\hline $\mathrm{Mn}$ & 0.50 & 0.57 & 0.85 & 0.47 & 0.36 \\
\hline Al & 0.47 & 0.55 & 1.20 & 1.05 & 1.13 \\
\hline Co & 0.0012 & 0.0008 & 0.0006 & 0.0012 & \\
\hline $\mathrm{Cu}$ & 0.24 & 0.024 & 0.026 & 0.067 & 0.26 \\
\hline $\mathrm{Sb}$ & - & - & - & - & 1.24 \\
\hline La & $\leq 0.0007$ & 0.0044 & 0.0015 & 0.0036 & $<0.001$ \\
\hline $\mathrm{Fe}$ & 0.16 & 0.58 & 0.44 & 0.35 & \\
\hline $\mathrm{Ca}$ & 9 & 9 & ح1 & 12 & 4 \\
\hline
\end{tabular}

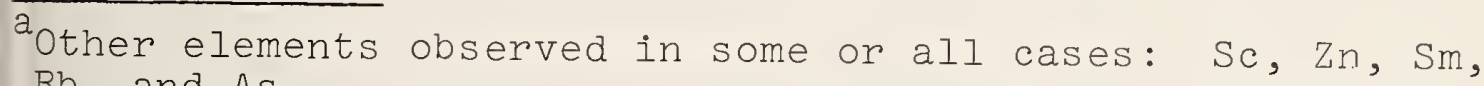
$\mathrm{Rb}$, and $\mathrm{As}$.

A consideration of the procedures and techniques used in making medieval glass provided a basis for the selection of useful comparisons and correlations of the various results in light of the original objectives. This information was obtained from the excellent series of papers on ancient glass and glassmaking by Turner [10] and the medieval treatise of Theophilus, "On Divers Arts" [11]. In the 
Middle Ages glass was made by mixing "ash" and "sand" with perhaps an additive or two to produce a desired color. It was not until the 18 th century that the differences between $\mathrm{K}$ and $\mathrm{Na}$ began to be known and, therefore, the ratio of the alkalis in medieval (and ancient) glasses would have been determined entirely by the ratio in the particular "ash" used. Medieval glass is, in general, characterized by a high $\mathrm{K}$ content which results from the use of vegetable ash, particularly beechwood ash, for glassmaking. Turner points out that the alkali content of such ash varies not only with the type of ash but also from place to place and from time to time depending on soil content, etc. He also points out that at various times ash from seacoast plants with high $\mathrm{Na}$ was popular.

In view of these considerations it seemed appropriate to calculate alkali ratios for the various glasses analyzed. These are listed in Table 16 and it can be seen that the glasses fall into groups with very different ratios, well outside the $\pm 10 \%$ limits mentioned above.

Table 16. Alkali ratios found in Medieval glasses.

Sample

Blue fragments

Bourges No. 4

Bourges No. 2

Bourges No. 3

"Bullseye" fragments

Bourges No. I

Bourges No. 5
\% Na

0.95

0.45

1.45

4.2

4.0

9.8

14.5
$\% \mathrm{~K}$

18.9

10.0

13.8

3.8

3.5

$<2$

$<2$
$\underline{\mathrm{K} / \mathrm{Na}}$

19.9

22.2

$9 \cdot 5$

0.90

0.88

$<0.2$

$<0.14$

Turner also mentioned that beechwood ash has an unusually high Mn content so that the source of the Mn in the glasses might again be the "ash" rather than a decolorizing agent or 
other additive. In this case the Mn-alkali ratios might also be significant. Table 17 gives these ratios for the same glasses and division into the same groups can be made, although the agreement is less striking than in the case of the $\mathrm{K} / \mathrm{Na}$ ratios.

Table 17. Manganese/sodium ratios found in Medieval glasses.

\begin{tabular}{lccc}
\multicolumn{1}{c}{ Sample } & $\frac{\% \mathrm{Na}}{0.95}$ & $\frac{\mathrm{Mn}}{1.08}$ & $\frac{\mathrm{Mn} / \mathrm{Na}}{1.14}$ \\
Blue fragments & 0.95 & 0.47 & 1.04 \\
Bourges No. 4 & 0.45 & 0.57 & 0.39 \\
Bourges No.2 & 1.45 & 0.85 & 0.20 \\
Bourges No. 3 & 4.2 & 0.40 & 0.10 \\
"Bullseye" fragments & 3.9 & 0.50 & 0.051 \\
Bourges No. 1 & 9.8 & 0.36 & 0.025. \\
Bourges No. 5 & 14.5 &
\end{tabular}

$\mathrm{Rb}$ was detected in all three of the high-K glasses (Bourges no. 2, no. 4 and the blue fragments) and, as can be seen from tables 14 and 15, glasses analyzed can be divided into almost identical groups on the basis of the La concentrations. Much more work needs to be done on the treatment of the data to gain the maximum amount of information, but before attempting this, data should be obtained on samples whose provenance is known much more accurately than is the case for the samples available for this study.

d. Analysis of Panes from Medieval Windows

The Gellatly Collection of the National Collection of Fine Arts, Smithsonian Institution, contains a group of stained glass windows whose attribution is French, 14 th Century. Mr. Charles Olin was studying one of these windows "The Angel of the Passion," which is shown in figure 15. He observed that although the three blue panes on the right of the window appeared similar in color under ordinary light, under inframed light the lower pane appeared to be different 


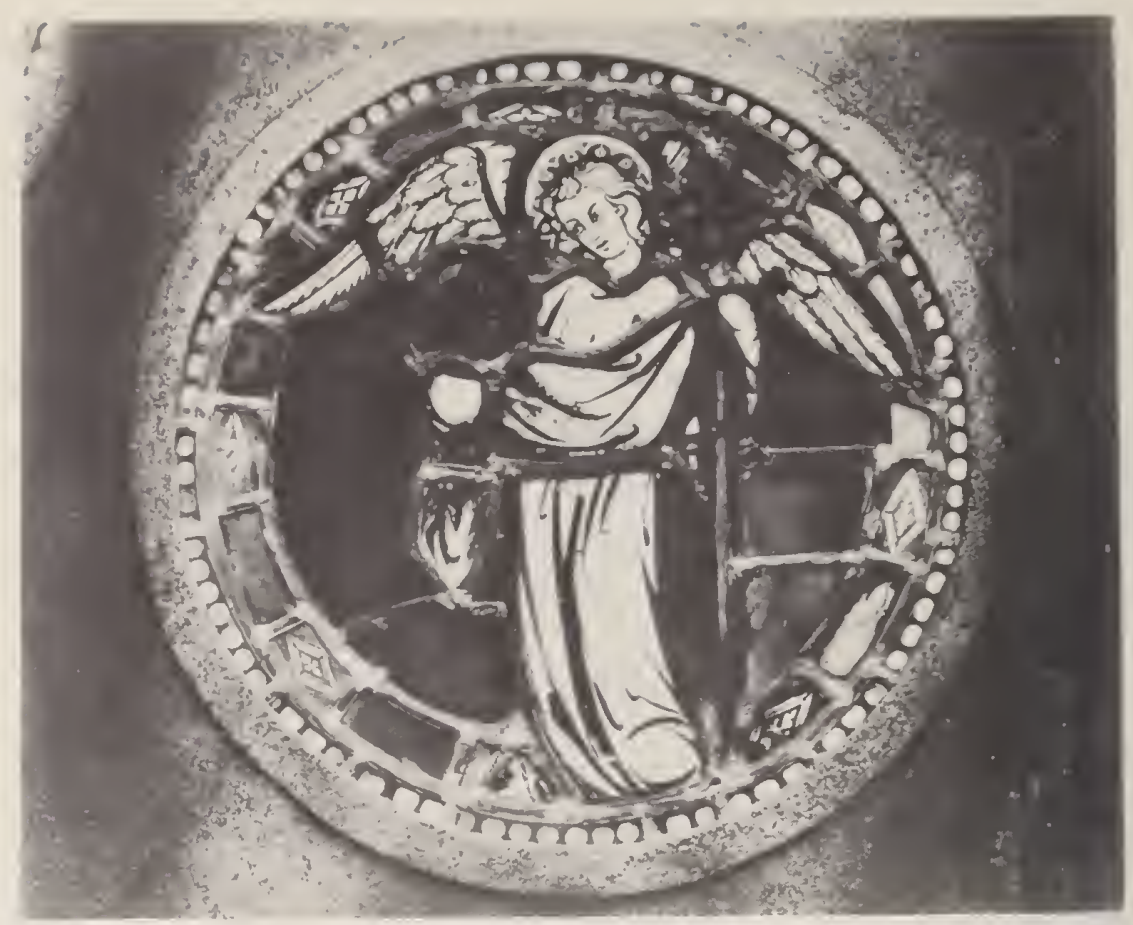

Figure 15. French 14th Century circle of stained glass Angel of the Passion. (Photograph courtesy of the National Collection of Fine Art,s, Smithsonian Institution.)

from the other two. By lifting the leading around the panes and very carefully chipping the edges he was able to remove small samples from each of the three panes. Two 10-mg and one 50-mg samples of each pane were analyzed by the neutron activation procedure described above and the results are shown in table 18. It is clear that the middle pane, not the lower, is very markedly different from the other two. The upper and lower panes are more like each other than they are like the middle pane, but they are far from identical. The difference in the appearance under infra-red light may possibly be explained by the substantially lower cobalt concentration in the lower pane relative to the other two, since it is this constituent that produces the blue color. In any case the results show very clearly the risks in drawing conclusions about an entire window from the analysis of a single pane or by a single technique. 
Table 18. Results of activation analysis of Medieval window, \%.

\begin{tabular}{|c|c|c|c|}
\hline lement & Upper Pane & Middle Pane & Lower Pane \\
\hline $\mathrm{Na}$ & $0.302\left\{\begin{array}{l}0.293 \\
0.294 \\
0.300 \\
0.319\end{array}\right.$ & $2.49\left\{\begin{array}{l}2.40 \\
2.50 \\
2.56\end{array}\right.$ & $0.207\left\{\begin{array}{l}0.212 \\
0.184 \\
0.224\end{array}\right.$ \\
\hline K & $17.24\left\{\begin{array}{l}16.73 \\
16.76 \\
17.25 \\
18.22\end{array}\right.$ & $11.33\left\{\begin{array}{l}11.65 \\
11.48 \\
11.86\end{array}\right.$ & $20.49\left\{\begin{array}{l}19.92 \\
21.21 \\
20.33\end{array}\right.$ \\
\hline Mn & $1.09\left\{\begin{array}{l}1.11 \\
1.06\end{array}\right.$ & $1.14\left\{\begin{array}{l}1.18 \\
1.11\end{array}\right.$ & $0.79\left\{\begin{array}{l}0.8 .6 \\
0.757\end{array}\right.$ \\
\hline $\mathrm{Cu}$ & $\begin{array}{l}0.129 \\
0.127\end{array}$ & $\begin{array}{l}0.462 \\
0.445\end{array}$ & $\begin{array}{l}0.131 \\
0.114\end{array}$ \\
\hline $\mathrm{Al}$ & $\begin{array}{l}1.48 \\
1.45\end{array}$ & $\begin{array}{l}0.517 \\
0.501\end{array}$ & 0.878 \\
\hline $\mathrm{Co}$ & $\begin{array}{l}0.0705 \\
0.0717\end{array}$ & 0.0920 & 0.0441 \\
\hline $\mathrm{Ca}$ & 13 & 6 & 8 \\
\hline La & $\begin{array}{l}0.00266 \\
0.00277\end{array}$ & 0.00412 & 0.00207 \\
\hline $\mathrm{Sb}$ & $\begin{array}{l}0.00436 \\
0.00477\end{array}$ & 0.0123 & 0.00230 \\
\hline $\mathrm{K} / \mathrm{Na}$ & 57 & 4.4 & 104 \\
\hline $\mathrm{Mn} / \mathrm{Na}$ & 3.5 & 0.45 & 3.9 \\
\hline
\end{tabular}

In, $\mathrm{Zn}$ and $\mathrm{Sc}$ present in all panes, Ta present in lower pane only.

(J. S. Olin, E. C. Miller, and B. A. Thompson)

2. The Determination of Sulfur Using Secondary Reactor Tritons

The determination of sulfur is of great importance in the metallurgical, biological, and environmental sciences. The most widely used method for this determination utilizes bomb combustion followed by volumetric or gravimetric analysis. With complete combustion, this technique maintains the accuracy of gravimetric or volumetric analysis, but is time- 
consuming and does not lend itself to large numbers of samples.

Tritons of $2.74 \mathrm{MeV}$ produced in a reactor via the ${ }^{6} \mathrm{Li}(\mathrm{n}, \alpha){ }^{3} \mathrm{H}$ reaction have been used for the determination of oxygen utilizing the ${ }^{16} \mathrm{O}(t, n)^{18}$ F reaction [12]. However, the method reported has never been totally successful for low concentrations of oxygen due to the large oxygen contamination in salts of lithium-6 used as sources of tritons. In the case of sulfur, this type of interference should be negligible due to the low sulfur content in isotopically enriched lithium-6 salts .

The use of the ${ }^{32} \mathrm{~S}(t, n)^{34 \mathrm{~m}}$ reaction was investigated [13] and more recently Albert et al. suggested the analytical utility of the reaction because of its specificity below $213.5 \mathrm{MeV}$ and the high natural abundance of sulfur-32 [14]. However, this reaction has not yet been analytically exploited because of the lack of accelerators with the authorization to accelerate tritium.

An activation analysis technique using reactor produced secondary tritons to induce the ${ }^{32} \mathrm{~S}(t, n){ }^{34 \mathrm{~m}} \mathrm{Cl}$ reaction has been investigated for the rapid determination of sulfur in fuel oils. In this work, the $2.78 \mathrm{MeV}$ tritons were used as they are slightly above the $2.38 \mathrm{MeV}$ threshold calculated for the ${ }^{32} \mathrm{~S}(t, n){ }^{34 \mathrm{~m}} \mathrm{Cl}$ reaction. The calculated reaction threshold was made using a non-relativistic charge interaction equation [15]. The useful range of the $2.78 \mathrm{MeV}$ triton in fuel oil is about $2 \times 10^{-3} \mathrm{~cm}$. Thus, a thin film of oil coating a lithium fluoride crystal will stop the triton. However, to use solid lithium fluoride coated with oil, the average particle size of the lithium fluoride must be a constant.

\section{a. Experimental}

Approximately $25 \mathrm{mg}$ of solid lithium fluoride was weighed into a quartz ampoule, two drops of oil allowed to coat the 
salt, and the ampoule sealed. The samples were irradiated for 15 seconds in the RT-4 facility of the NBS Reactor at a thermal neutron flux of $\sim 1.3 \times 10^{13} \mathrm{n} \cdot \mathrm{sec}^{-1} \mathrm{~cm}^{-2}$. After irradiation, the samples were washed into a separatory funnel with $4 \mathrm{cc}$ of benzene. The ionic chlorine $34 \mathrm{~m}$ was extracted with water containing $25 \mathrm{mg}$ of inactive chloride carrier. The chloride ion was precipitated as silver chloride, washed and counted for 2 half-lives of the chlorine-34m. The benzene phase was washed into a 4 dram vial, brought to $6.0 \mathrm{ml}$ volume and counted in a 5 in. $x 4$ in. well NaI (TI) crystal.

b. Results and Discussion

Residual fuel oils of known sulfur content (SRMs 1621 and 1622) were prepared and analyzed by the above procedure. Samples of SRM 1622 were analyzed using different weights of

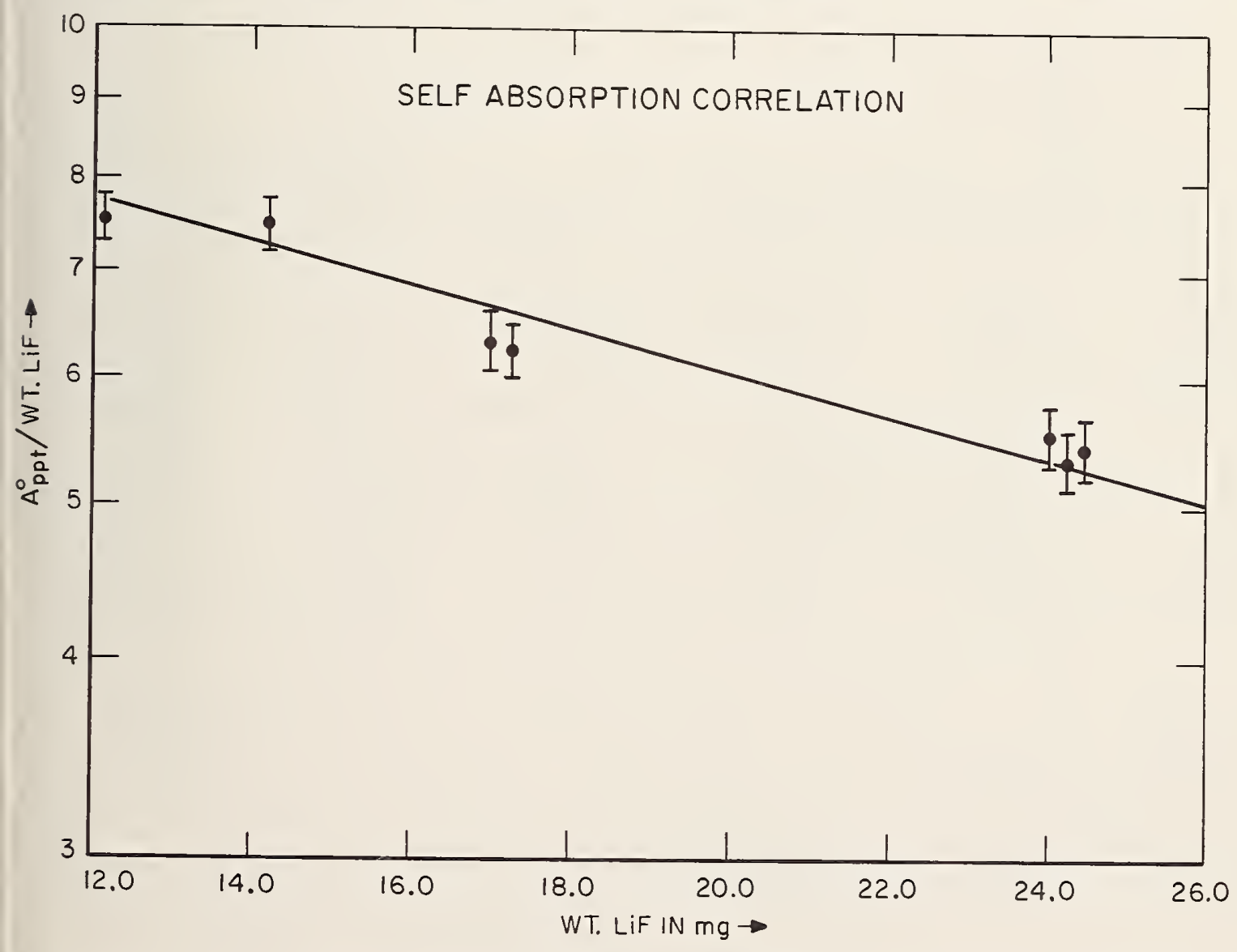

Figure 16. Correlation between weight and neutron selfabsorption of lithium fluoride. 
${ }^{6}$ LiF to obtain a correlation between weight and self absorption of the lithium fluoride (fig. 16). This correlation was used to correct the countrates of the silver chloride precipitates and these results used for analysis. The results are given in table 19.

Since the benzene phase results were not consistent, the lithium fluoride was ground in a ball mill to reduce the particle size and triturated in a low sulfur content oil. A suspension of $100 \mathrm{mg}$ of LiF per $\mathrm{ml}$ of oil was mixed with an equal weight of standard sulfur oil and analyzed by the previously mentioned procedure. The initial experiments showed no neutron self-absorption if less than $0.25 \mathrm{~g}$ of oil mix was used. Analytical results are currently being obtained.

Table 19. Results of sulfur analysis.

\begin{tabular}{|c|c|c|c|c|c|}
\hline Sample & $\mathrm{A}^{\circ \mathrm{a} p \mathrm{pt}}$ & $A^{\circ}$ Benzene & $\%$ Eff. & $\begin{array}{l}\text { Wt } \mathrm{LiF} \\
\text { in Mg }\end{array}$ & ${ }_{\%}^{\text {Using }} A^{\circ} p p t$ \\
\hline $1622-1$ & 186.2 & 153.6 & 56.1 & 12.09 & sta. \\
\hline $1622-2$ & 277.7 & 240.7 & 53.8 & 24.00 & Std. \\
\hline $1621-1$ & 107.1 & 120.7 & 46.1 & 17.22 & 0.98 \\
\hline $1621-2$ & 106.2 & 135.2 & 44.1 & 14.16 & 1.06 \\
\hline $1621-3$ & 108.0 & 137.5 & 44.1 & 16.98 & 1.00 \\
\hline $1621-4$ & 134.2 & 140.8 & 48.8 & 24.42 & 1.05 \\
\hline $1621-5$ & 130.5 & 124.7 & 51.2 & 24.24 & 1.02 \\
\hline Average & & & & & $1.02 \pm .04$ \\
\hline Certified & Value & & & & $1.05 \pm .02$ \\
\hline
\end{tabular}


3. Analysis for Iodine in Protein Solutions by Neutron Activation Analysis

In collaboration with scientists of the thyroid group in the Clinical Endocrinology Branch of the National Institute of Arthritis and Metabolic Diseases, preliminary investigations were made into the possibility of using neutron activation analysis for the determination of low levels of iodine. The samples of interest were dialyzed protein solutions, both normal and iodinated. Expected iodine levels ranged from $10 \mathrm{ng} / \mathrm{ml}$ to $1000 \mathrm{ng} / \mathrm{ml}$ ( 0.01 to $1 \mathrm{ppm}$ ).

\section{a. Experimental}

Initial tests were made on protein solutions with varying amounts of added iodide. Reagent grade potassium iodide was used as the standard, with care taken to prevent oxidation of the iodide to free iodine during the analysis.

A procedure was developed which allowed rapid analysis, of samples ( $20 \mathrm{~m}$ each, including irradiation and counting) ranging from $5 \mathrm{ng} / \mathrm{ml}$ to $10 \mathrm{mg} / \mathrm{ml}$ or higher. All the samples and standards were encapsulated in specially cleaned, heat sealed medical grade polyethylene tubing. The procedure developed included irradiation in RT-3 of the NBSR for $5.0 \mathrm{~m}$ (thermal neutron flux $\sim 5 \times 10^{13} \mathrm{n} \cdot \mathrm{cm}^{-2} \mathrm{sec}^{-1}$ ), removal from the polyethylene rabit, washing the outside of the polyethylene container in dilute $\mathrm{HnO}_{3}(l: 1)$, rinsing several times in distilled $\mathrm{H}_{2} \mathrm{O}$, and then drying, positioning in the counting holder, and counting for $10.0 \mathrm{~m}$ with a $60 \mathrm{~cm}^{3} \mathrm{Ge}(\mathrm{Li})$ detector and 1024 channel PHA. The counting geometry was varied from $1.25 \mathrm{~cm}$ to $20 \mathrm{~cm}$ from the detector to compensate for differences in radioactivity levels between samples. All geometry positions were calibrated with iodine-128 standards to determine efficiency factors. Flux normalization of the various irradiations were made with copper foil flux monitors.

\section{b. Results and Discussion}

The results of analysis for the preliminary tests yielded the expected straight line relationship between various iodine concentrations and the $0.441 \mathrm{MeV}$ radioactivity. The 
lack of interference using this gamma ray peak was verified by half-life determinations. In addition, the half-life determination verified that no significant amount of iodine escaped by diffusion through the polyethylene during this period. Considering that free iodine easily diffuses through polyethylene, and the oxidizing nature of ionizing radiation, this question was a real concern.

Blank levels were determined for the polyethylene tubing and for the dialyzing solution, and were found to be approximately $5 \mathrm{ng} / 0.5 \mathrm{ml}$, respectively. Actual samples received varied from $18 \mathrm{ng} / 0.5 \mathrm{ml}$ to $17.7 \mu \mathrm{g} / 0.1 \mathrm{ml}$.

The results obtained using neutron activation analysis agreed well with the values obtained from wet chemical techniques for the high iodine level solutions. The low level iodine samples could not be checked this way, since for these levels the wet chemical technique was not reproducible.

(D. A. Becker and W. D. Kinard)

4. Lead Alloy Flux Monitors for Neutron Activation Analysis

Since the NBSR came into routine use for neutron activation analysis, the high neutron flux available has encouraged the development of a variety of materials as flux monitors for long irradiations in high flux positions. In particular, alloys of lead with a number of different elements is in progress.

The requirements for flux monitors for high precision and accuracy in activation analysis has been discussed elsewhere $[16,3]$. Examination of commonly available elements for their neutron activation and decay characteristics as well as their metallurgical properties reveal several that could be conveniently used. Lead was chosen as the dilutant due to its lack of gamma ray producing radioactive products after neutron irradiation and because of its density and malleability. Dilute alloys of lead with many elements 
should remain ductile to allow rolling into foil form. After production, these foils must then be examined for their suitability as flux monitors.

The elements which have been alloyed with lead to produce foils are copper, gold, zinc, and indium. Selenium was attempted but has not yet been alloyed before combustion occurs. At present, only the copper-lead alloy and the goldlead alloy (0.1\% element in lead) have been tested for homogeneity. Both of these elements seem to behave quite well, yielding with care a homogeneous melt which can then be rolled into 25 mil foil ( $f i g . ~ 17)$ and cut into small ( $25 \mathrm{mg}$ ) pieces for use as flux monitors. While reagent grade lead was used for testing purposes, only $99.999 \%$ purity material was used for the final products. The homogeneity of actual $0.1 \%$ copper-lead alloy flux produced in this laboratory averaged $\pm 1.1 \% \mathrm{~S}_{d}$, about twice that of pure copper

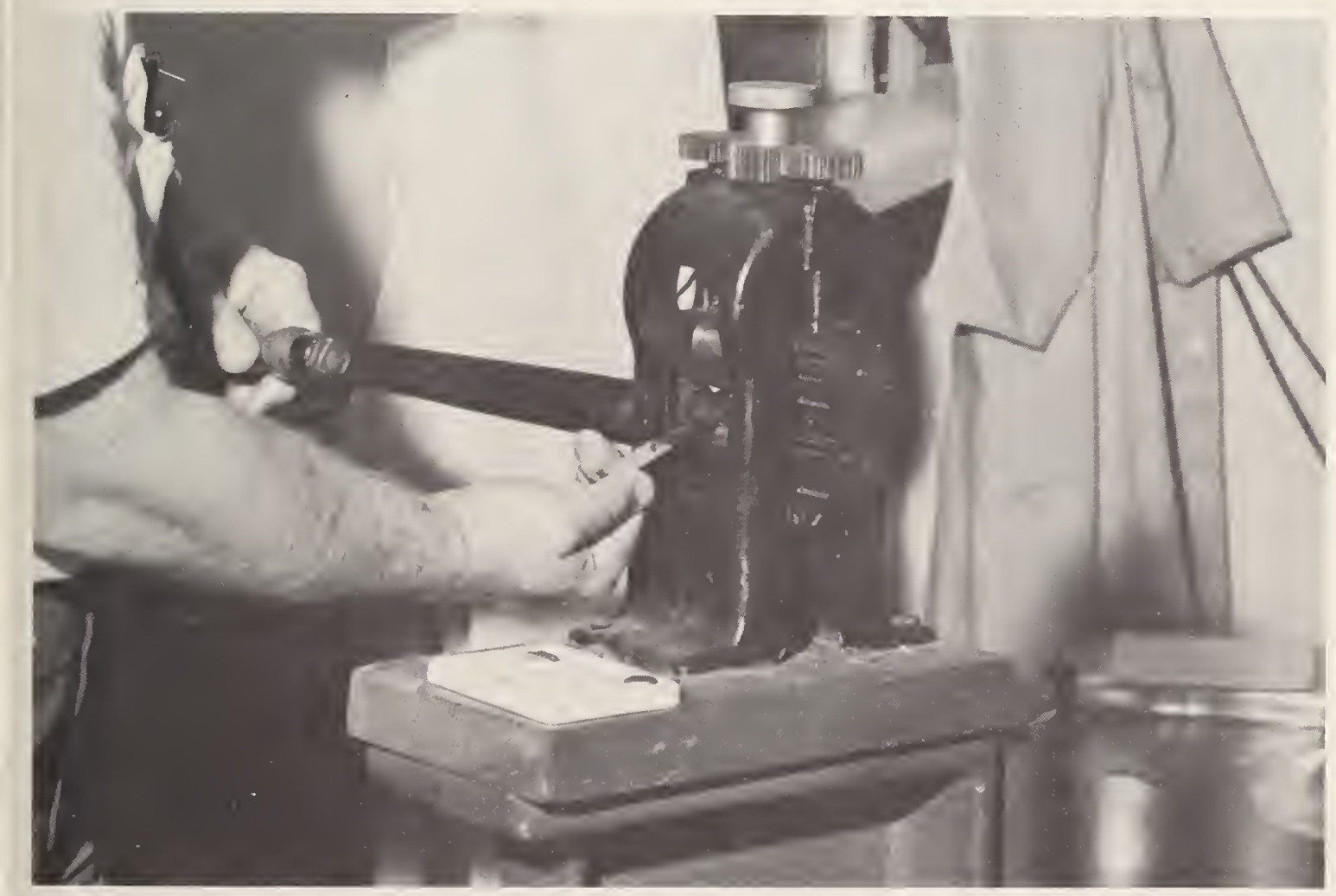

Figure 17. Lead alloy ingot being rolled into a thin foil. 43 
flux monitor foils [3]. The homogeneity of gold-lead alloy foils was slightly higher, at $\sim \pm 2 \%$.

Efforts have been made to interest a private company in producing the copper-lead alloy on a large scale for flux monitor use. However, several attempts by this company failed to produce a foil with acceptable specifications. Additional attempts are presently being made either to find a different alloy which can be mass produced, or to find another method of producing an acceptable copper-lead alloy.

(D. A. Becker and P. D. LaFleur)

5. The Extraction of Metals With Bis(2-ethylhexyl) Orthophosphoric Acid

The solvent extraction studies using bis(2-ethylhexyl) orthophosphoric acid (HDEHP) as the extractant which were described previously [17] have been largely concluded. The extraction of 53 elements was studied from 1 to IIM solutions of hydrochloric, perchloric and nitric acids into $0.75 \mathrm{M}$ HDEHP in petroleum ether. The extraction curves of these elements are shown in figures 18, 19, and 20. At the present time no more data has been acquired as to the mechanism of extraction using this reagent, but we are planning to investigate this mechanism in the future. At the present time effort is going into the application of these extractions to group and individual element separations for use with activation analysis, an area which we believe will be most fruitful.

(I. H. Qureshi and P. D. LaFleur) 

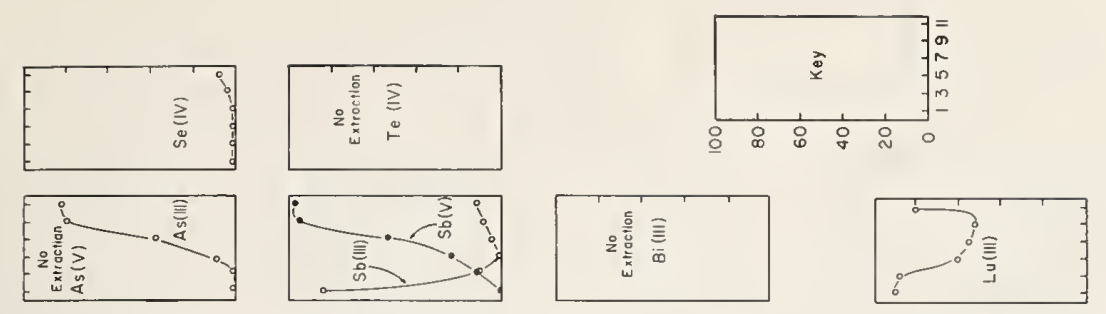

0
0
0
0
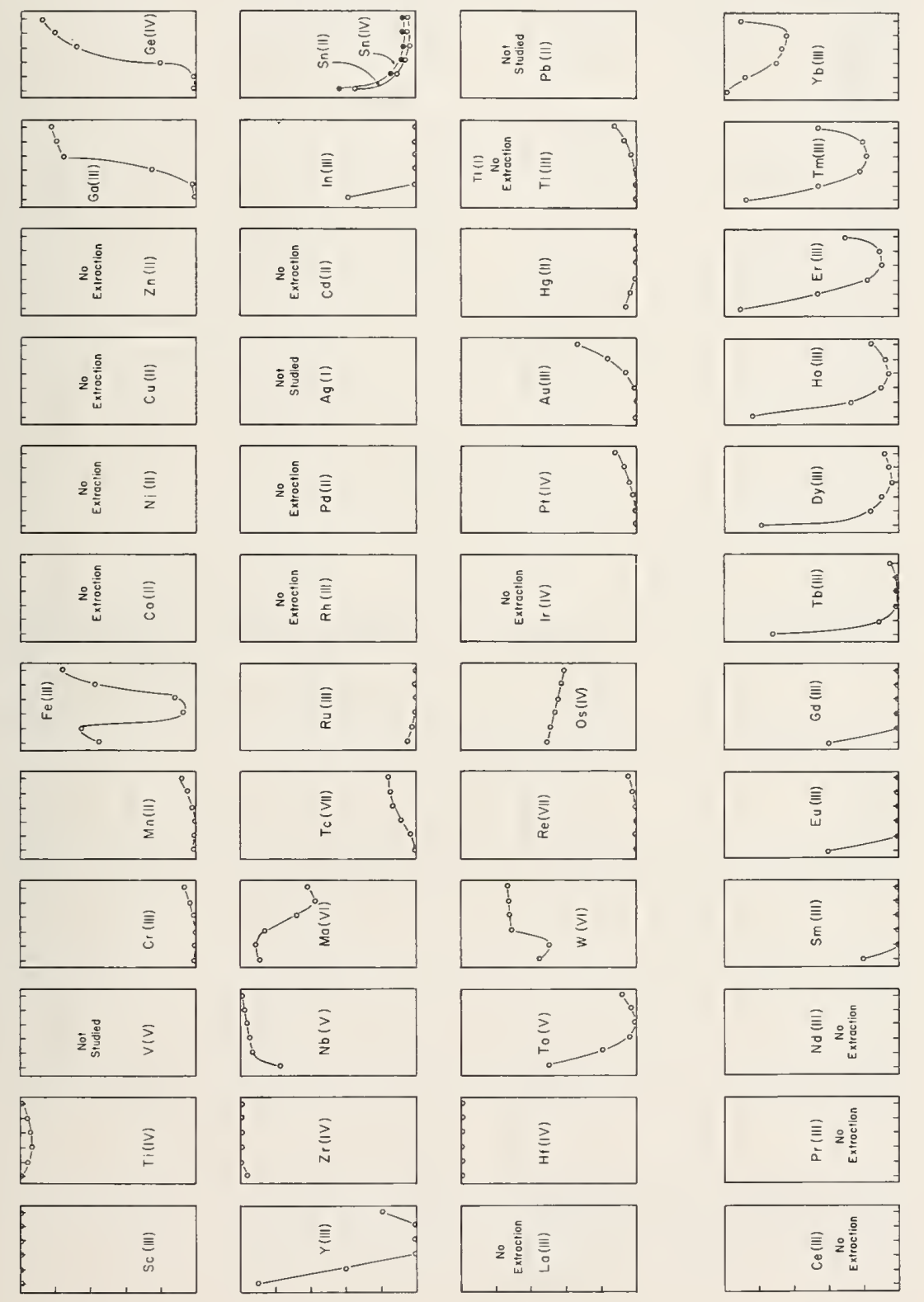

๑ $\lrcorner コ \forall y \perp x \exists \perp N \exists ว y \exists d$ 

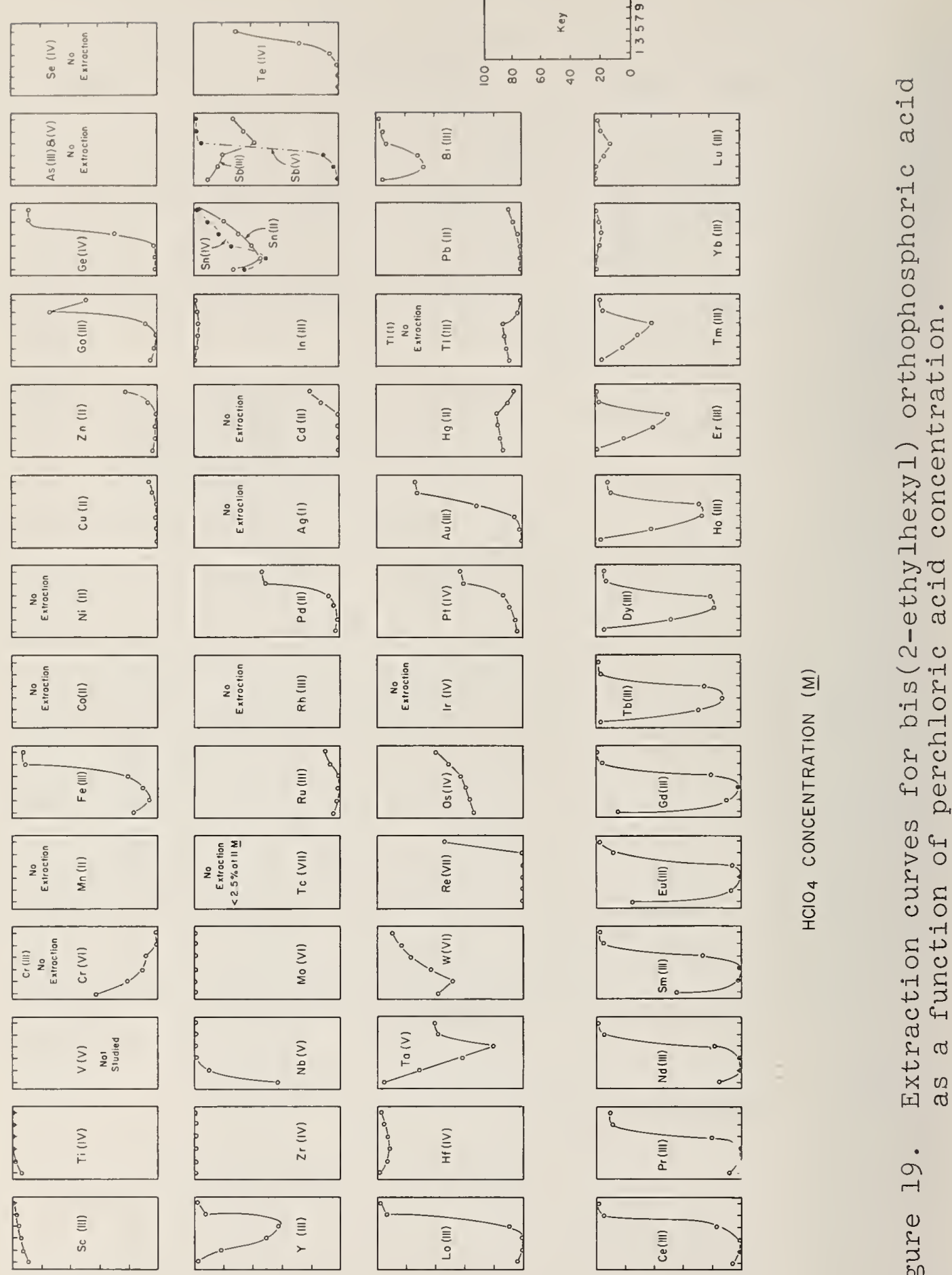

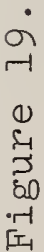

$0 \exists \perp \supset \forall Y \perp X \exists \perp N \exists \supset Y \exists d$ 


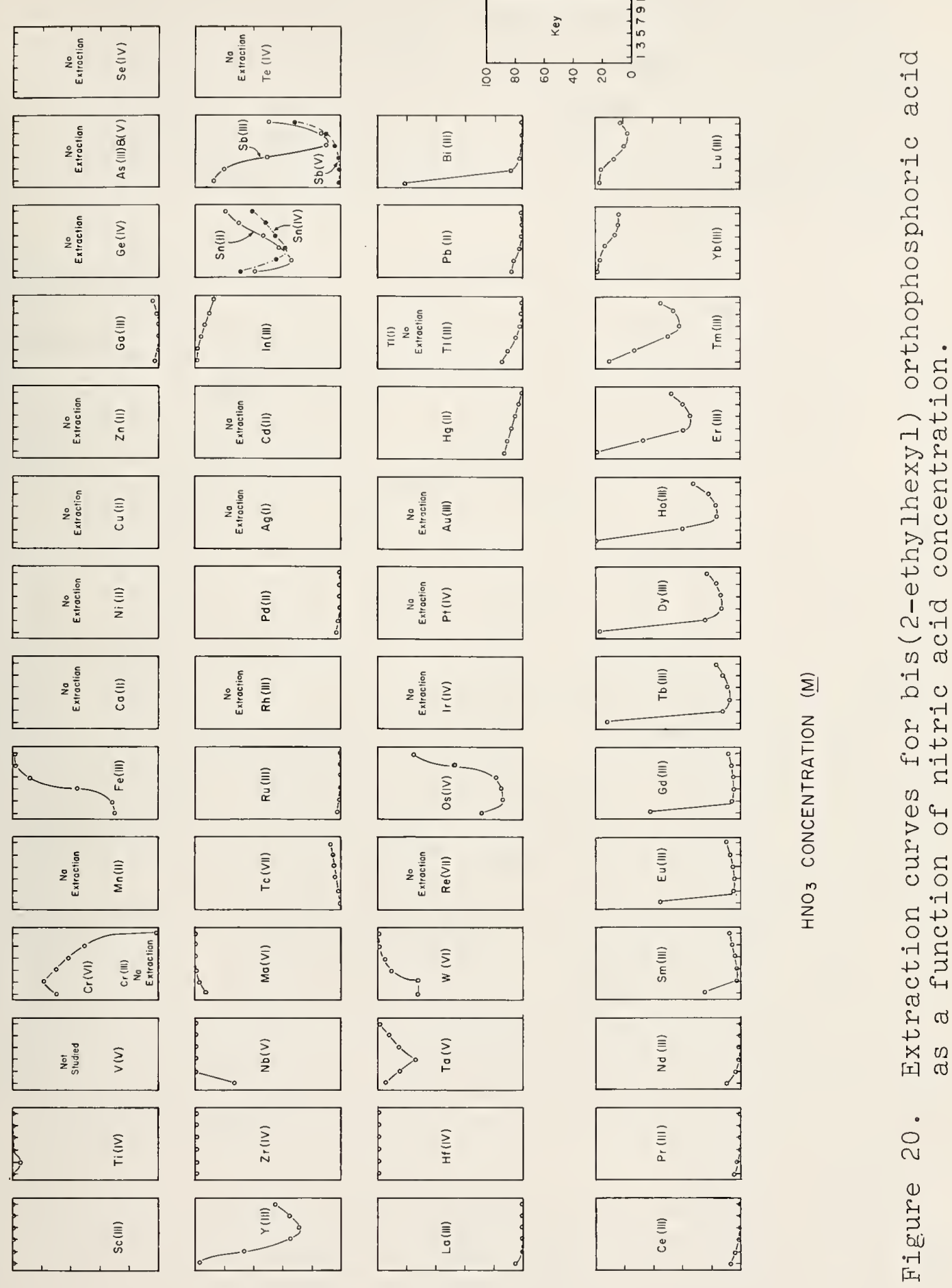

0 $\triangle \perp \forall \forall \perp X \exists \quad \perp N \exists J Y \exists d$ 
6. The Determination of Short-lived Trace Impurities in Sodium Metal by Neutron Activation Analysis

The determination of trace elements in sodium has recently received much interest because of the use of sodium in liquid metal cooled reactor technology. For liquid sodium reactors the determination of corrosion products demands state of the art analytical techniques.

To measure the extent of corrosion in the coolant lines of liquid-metal-cooled fast reactors, it is necessary to measure the build-up of the elements from the stainless steel piping present in the coolant as a function of operating time. Because of the high chemical reactivity of sodium metal, it is very difficult to maintain the integrity of the sample from the time it is drawn until it is analyzed.

Activation analysis has been largely ignored as a technique for analysis of a sodium matrix because of the intense activity of the sodium-24 created during irradiation. The analysis of elements producing short-lived ( 15 hrs) isotopes is especially difficult. The high energy gamma rays and its long half-life makes nondestructive analysis impossible. Destructive analysis requires a number of time consuming radiochemical separations. The time required to allow the matrix to decay is especially critical for the determination of elements such as $\mathrm{Mn}, \mathrm{Cu}$, and $\mathrm{W}$, etc. since all have halflives of 24 hrs or less. In addition, waiting a week or more to begin processing the samples after irradiation is usually undesirable. Because of the radiation hazards involved with handling the samples, involved chemical manipulations are very unattractive.

Recently Girardi and Sabbioni [18] reported the use of hydrated antimony pentoxide (HAP) to remove radiosodium from biological samples. Decontamination factors of $\geq 10^{10}$ were reported for sodium removal from $12 \mathrm{~m}$ HCl solutions. The only other element of the 60 elements they studied retained 
by the HAP was Ta, which was also quantitatively removed. The activation analysis of glass samples, containing $14 \%$ $\mathrm{Na}_{2} \mathrm{O}$, indicated that HAP was indeed as effective for radiosodium removal as was indicated by Girardi and Sabioni. Based on these experiments HAP was used to remove the matrix activity to allow the analysis of short-lived trace impurities in sodium metal.

\section{Experimental}

Samples of sodium weighing 100 to $250 \mathrm{mg}$ were encapsulated in medical grade polyethylene or quartz tubing and irradiated for 12 minutes to 2 hrs at a flux of $\sim 5 \times 10^{13}$ $\mathrm{n} . \mathrm{cm}^{-2} \mathrm{sec}^{-1}$ in the pneumatic tube facilities of the NBSR. After irradiation, the sample was lightly etched in ethanol and then dissolved in a 60\% ethanol-40\% water mixture. The sample was evaporated to dryness with a heat lamp, carefully taken up with concentrated $\mathrm{HCl}$, and diluted to $25 \mathrm{ml}$ in a volumetric flask. A $1.0 \mathrm{ml}$ aliquot is taken for subsequent determination of the sodium content and therefore the total amount of sodium taken for analysis. The remaining solution is passed through a l-cm diameter column containing $\sim 5 \mathrm{~g}$ of HAP. After the entire solution has passed through the HAP, the column is washed with 10 to $15 \mathrm{ml}$ of $12 \mathrm{M} \mathrm{HCl}$, and the wash combined with the main sample. The sample was then counted using a $47 \mathrm{cc}$ Ge(Li) detector in conjunction with a 4096 channel analyzer. In the spectrum energy range 0-3.5 $\mathrm{MeV}$, one could easily see ${ }^{64} \mathrm{Cu},{ }^{56} \mathrm{Mn},{ }^{122} \mathrm{Sb},{ }^{87} \mathrm{Br}$, ' and ${ }^{42} \mathrm{~K}$. As an example of the decontamination achieved with HAP, only a small peak from ${ }^{24} \mathrm{Na}$ could be seen in the eluate from a column having a radiation level of $4 \mathrm{R} / \mathrm{hr}$. The manipulations involved are readily adaptable to remote handling.

To see if these elements represented the bulk of the matrix, several etches were made and it was found that the impurities observed were representative of the entire sample.

(T. E. Gills and P. D. LaFleur) 
1. Biological Standard Reference Materials: Orchard Leaves

After several years of looking into the need for and preparation of a number of biological Standard Reference Materials: [19] an NBS Standard Reference Material, Orchard Leaves, is now in the process of being analyzed and certified (table 20). At present, six different botanical materials have been acquired (orchard leaves, tomato leaves, citrus leaves, alfalfa, pine needles and aspen chips) of which one, orchard leaves, has been tested for homogeneity, radiation sterilized, packaged: and is undergoing analysis. This one botanical material will be certified for major, minor and trace elemental content. In addition to these botanical materials, efforts are being made to obtain large samples of lyophilized beef liver and lyophilized bovine serum. These materials will also be prepared and analyzed for elemental content, and issued as Standard Reference Materials.

The total number of elements in these Biological SRM's which will finally be certified is still uncertain. At present, the elements most likely to be certified include nitrogen, phosphorus, potassium, calcium, magnesium, sodium, boron, iron, manganese, aluminum, zinc, copper, arsenic and iodine.

(P. D. LaFleur and D. A. Becker)

a. Determination of Moisture Content in SRM Orchard Leaves

In preparation for the analysis and certification of this material, a literature search revealed no standardized technique for drying a biological sample before analysis. Therefore, several of the different suggested techniques were tested in order that one or two effective drying methods could be suggested for this materials. The methods used included freeze drying (temperature not to exceed $50^{\circ} \mathrm{C}$ ) (fig. 2l), vacuum oven drying at $90^{\circ} \mathrm{C}$, oven (air) drying at $90^{\circ} \mathrm{C}$, and oven (air) drying at $135^{\circ} \mathrm{C}$. 
Table 20. NBS biological Standard Reference Materials.

Material

Botanical

Orchard leaves Citris leaves

Tomatoe leaves

Alfalfa

Pine needles

Aspen chips

Blood

Bovine serum

Tissue

Beef liver
Acquired Packaged Homogeneity Certified

$\begin{array}{llcc}\text { Yes } & \text { Yes } & \text { Yes } & \text { In process } \\ \text { Yes } & \text { Yes } & \text { In process } & \\ \text { Yes } & \text { No } & - & \\ \text { Yes } & \text { Yes } & \text { In process } & \\ \text { Yes } & \text { No } & - & \\ \text { Yes } & \text { No } & - & \end{array}$

In process

In process

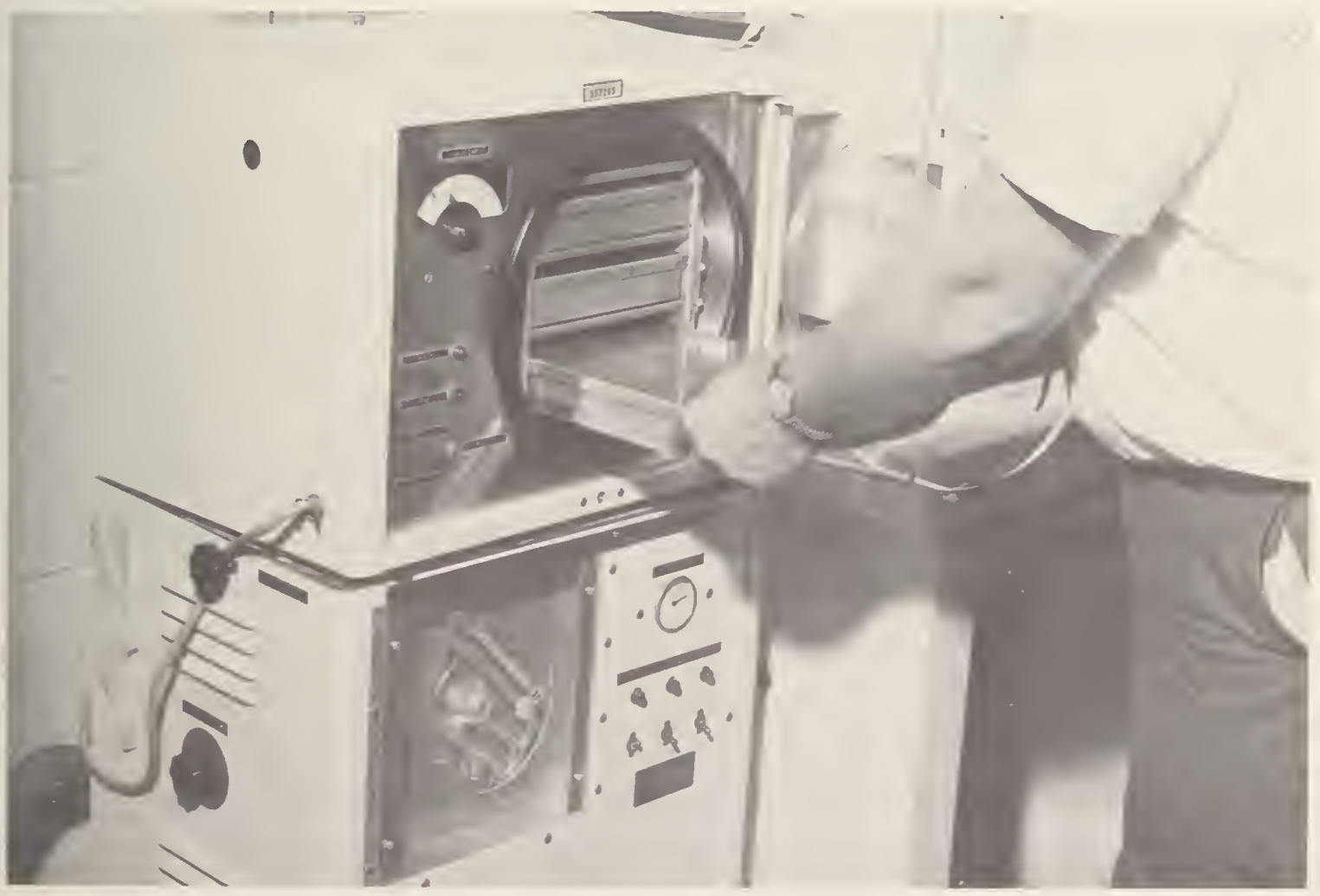

Figure 21. Freeze drying apparatus for the removal of moisture from biological samples. 
Table 2l. Moisture removal for orchard leaf samples.

\begin{tabular}{|c|c|c|c|c|c|}
\hline $\begin{array}{c}\text { Drying } \\
\text { Technique } \\
\end{array}$ & $\begin{array}{l}\text { Drying } \\
\text { Time } \\
\end{array}$ & Temperature $\left({ }^{\circ} \mathrm{C}\right)$ & $\begin{array}{l}\text { Visual } \\
\text { Change } \\
\end{array}$ & $\begin{array}{l}\text { Weight } \\
\text { Loss }(\%)\end{array}$ & $\begin{array}{l}\text { Average } \\
\text { Wt. Loss }(\%)\end{array}$ \\
\hline $\begin{array}{l}\text { Freeze } \\
\text { Drying }\end{array}$ & $24 h$ & $<50$ & None & $\begin{array}{l}3.39 \\
3.40\end{array}$ & 3.40 \\
\hline Vacuum oven & $24 h$ & 90 & None & $\begin{array}{l}3.05 \\
3.59\end{array}$ & $3 \cdot 32$ \\
\hline (Air) oven ${ }^{a}$ & $24 h$ & 90 & None & $\begin{array}{l}3.44 \\
3.53\end{array}$ & 3.48 \\
\hline (Air) oven ${ }^{a}$ & $24 h$ & 135 & Yes $^{b}$ & $\begin{array}{l}7.60 \\
7.29\end{array}$ & 7.45 \\
\hline $\begin{array}{l}\text { Dessicator } \\
{\left[\operatorname{Mg}\left(\mathrm{ClO}_{4}\right)_{2}\right]}\end{array}$ & $96 \mathrm{~h}$ & $20^{\circ} \mathrm{C}$ & None & $\begin{array}{l}1.87 \\
1.96 \\
2.05\end{array}$ & 1.96 \\
\hline
\end{tabular}

The results of these tests are given in table 21. Sample sizes were approximately 22 grams each. They were stored in dessicators for cooling prior to weighing. The results show that the first three drying techniques used, freeze drying, drying in a vacuum at $90^{\circ} \mathrm{C}$, and drying in an air oven at $90^{\circ} \mathrm{C}$, are all approximately equally effective without apparent loss of volatile materials. However, drying in an oven at $135^{\circ} \mathrm{C}$ obviously results in the loss of some of the sample, and is not recommended. Dessicator drying is not recommended due to the length of time required for complete drying. With only the dessicator, over half of the moisture is removed in four days. However, the remaining moisture would most likely require much longer to remove.

A few additional measurements were made on the rate of moisture pickup in an open laboratory room. These measurements revealed that in $20 \mathrm{~m}$ almost $0.3 \%$ moisture was regained. In $45 \mathrm{~m}$ standing, $0.7 \%$ moisture was regained, or almost one quarter of the total moisture lost on drying. The foregoing 
results emphasize that these samples or any dried samples should not be exposed to the open air for long without redrying the samples.

(D. A. Becker and P. D. LaFleur)

b. Homogeneity of Botanical Standard Reference Material: Orchard Leaves

The first of these materials to be studied was the sample of dried powdered orchard leaves. Before proceeding with the analyses it was necessary to show that the distribution of elements in the $220 \mathrm{~kg}$ of sample was homogeneous. other Sections within the Analytical Chemistry Division also participated in this study. The Activation Analysis Section studied the distribution of Mn in the samples. The sampling technique used was designed by Dr. J. Mandell, Statistical Consultant to the Institute for Materials Research, to satisfactorily sample the 10 barrels containing the sample.

\section{(1) Experimental}

The samples as received from OSRM were in tightly closed bottles. A portion of each sample was transferred to a clean plastic tray and freeze-dried over night. After removal from the freeze dryer, the samples were stored in several freshly cleaned dissicators over calcium sulfate. Individual samples of $250 \mathrm{mg}$ were accurately weighed and prepared for irradiation. SRM steel No. IOle, containing 1.77\% manganese, was used as the standard. The samples were irradiated for 10 seconds in the NBSR RT-4 pneumatic tube facility. Counting was delayed for $\sim 2.5$ hours to allow $27 \mathrm{Mg}\left(t_{1 / 2}=9.5 \mathrm{~min}\right)$ to decay. Samples and standards were counted with identical geometries on a large volume Ge(Li) detector coupled to a 4096 channel PHA. After corrections for decay and flux normalization, the manganese concentrations were calculated. 


\section{(2) Results and Discussion}

Results of the individual analyses are shown in tables 22 and 23. The average of all the samples is shown in table 24.

Table 22. Manganese homogeneity: orchard leaves.

Sample No. Sample Position

$\begin{array}{ll}0-2 & \text { P - top } \\ 0-2 & \text { Middle center } \\ 0-2 & \text { Bottom center } \\ 0-2 & \text { Q - middle } \\ 0-2 & \text { Right bottom } \\ 0-2 & \text { Top center } \\ 0-2 & \text { Overall average } \\ 0-3 & \text { Top center } \\ 0-3 & \text { Right bottom } \\ 0-3 & \text { Bottom center } \\ 0-3 & \text { Q - middle } \\ 0-3 & \text { P - top } \\ 0-3 & \text { Middle center }\end{array}$

$0-3$

$0-3$

$0-5$

$0-5$

$0-5$

$0-5$

$0-5$

$0-5$

$0-5$

Right bottom
Manganese Conc.

(

\section{Average}

$104 ., 103$

103.

$98.4,102$.

100.

$94.2,99.8$

97.0

$100 ., 101$.

100 .

101., 100.

100 .

$100 ., 106$.

103.

\section{$101 . \pm 1.9$}

101., 99.6

100 .

$107 ., 104$.

106.

$102 ., 98.6$

100 .

$103 ., 96.3$

99.6

103., 97.4

100 .

108., 99.6

104.

\section{$102 . \pm 2.8$}

$99.5,105$.

102

101., 107

104

$98.7,105$.

102.

101., 99.0

100.

$103 ., 99.2$

101.

$98.6,97.4$

98.0

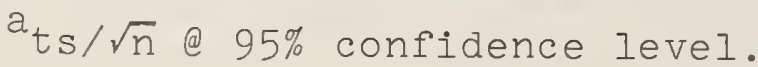


Table 23. Manganese homogeneity: orchard leaves.

Sample No. Sample Position

$0-6$

$0-6$

$0-6$

$0-6$

$0-6$

$0-6$

$0-6$

$0-9$

$0-9$

$0-9$

$0-9$

$0-9$

$0-9$

$0-9$

$0-10$

$0-10$

$0-10$

$0-10$

$0-10$

$0-10$

$0-10$
Middle center

Q - midale

Top center

Bottom center

Right bottom

$P$ - top
Overall average ${ }^{a}$

P - top

Center top

Bottom center

Middle center

Q - Middle

Right bottom

Overall average ${ }^{a}$

Center top

Center bottom

Center midale

Q - middle

Right bottom

Overall average ${ }^{a}$
Manganese Conc.

\begin{tabular}{cc}
\hline (ppm) & Average \\
102., 95.4 & 98.7 \\
$97.0,95.7$ & 96.4 \\
$96.3,97.4$ & 96.8 \\
$93.9,99.7$ & 96.8 \\
$96.7,110$. & 103. \\
$100 ., 106$. & 103.
\end{tabular}

$99 \cdot 1 \pm 3 \cdot 3$

101.96 .4

$98 \cdot 7$

$122 . \mathrm{b}, 99.6$

99.6

$97.4,97.6$

97.5

$97.8,93.0$

95.4

$95.8,96.0$

95.9

$101 ., 94.1$

97.6

$97.2 \pm 1.7$

$99.1,99.0$

99.0

$97.9,97.1$

97.5

$97.4,95.3$

96.4

$98.9,96.9$

97.9

$97.7,94.0$

95.8

$98.1,94.8$

96.4

$97.2 \pm 1.2$

ats $/ \sqrt{n}$ at $95 \%$ confidence level.

This value rejected in subsequent calculations. 
Table 24. Combined averages for all containers.

Sample No.

$0-2$

$0-3$

$0-5$

$0-6$

$0-9$

$0-10$

Overall average (all samples) Range 93.0 - 108
Average Manganese Conc. $(\mathrm{ppm})^{\mathrm{a}}$

101 .

102.

101.

99.1

97.2

97.2

$99 \cdot 6 \pm 2.17$

$\overline{a_{t s} / \sqrt{n}}$ at $95 \%$ confidence level.

In general, the results indicated that, for manganese, these samples are homogeneous for $250 \mathrm{mg}$ to at least $\pm 3 \%$. All results were calculated on a dry weight basis.

(W. D. Kinard)

2. Determination of Cobalt, Silver, and Gold in SRM Glass by Neutron Activation Analysis

The determination of $\mathrm{Co}, \mathrm{Ag}$ and $\mathrm{Au}$ was required for certification of the SRM trace elements in glass. In many samples $\mathrm{Co}$ and $\mathrm{Au}$ can be determined nondestructively and $\mathrm{Ag}$ can be determined using a simple AgCl separation. However, in the case of the SRM glasses many uncommon elements were added and all the trace elements were added at about the same concentration levels. This resulted in different interferences than are ordinarily encountered and, thus, a different approach to the analysis.

The radionuclides produced by neutron activation of Co, $\mathrm{Ag}$ and $\mathrm{Au}$ all have half-lives long enough to permit decay of the very high ${ }^{24} \mathrm{Na}$ levels before analysis, and so the HAP separation for $\mathrm{Na}$ used in previous work [8] was not necessary. In this work the glass disks $(0.2-0.8 \mathrm{~g})$ were irradiated for 1 hour at a flux of $\sim 6 \times 10^{13} \mathrm{n} \cdot \mathrm{cm}^{-2} \cdot \mathrm{sec}^{-1}$ and set aside for 
7-10 days. After this time the principal radioactive species present include ${ }^{46} \mathrm{Sc},{ }^{198} \mathrm{Au},{ }^{182} \mathrm{Ta},{ }^{152} \mathrm{Eu},{ }^{140} \mathrm{La},{ }^{177} \mathrm{Lu}$, $153 \mathrm{Sm}$, and ${ }^{175} \mathrm{Yb}$. Thus, of the elements of interest, only Au could be determined nondestructively, even in principle; but, because the actual Au concentration level was in some cases only 5-10\% of the nominal ( 5 ppm instead of $50 ; 20$ ppm instead of 500), separation of the Au was necessary to obtain the degree of precision required for certification. Because of the extremely high ${ }^{24} \mathrm{Na}$ levels present immediately after irradiation, and the resulting high radiation fields (>100 $\mathrm{r} / \mathrm{hr}$ per sample at $\mathrm{l} \mathrm{cm}$ ) encountered in transferring the irradiated samples to storage containers, it was desirable to minimize the number of irradiations by determining all the elements of interest in the same sample. The following procedure was therefore developed and utilized.

The irradiated glasses were dissolved in $\mathrm{HF}_{-} \mathrm{HClO}_{4}$ and carried to fumes of $\mathrm{HClO}_{4}$. The residue was taken up in $6 \mathrm{~N}$ $\mathrm{HCl}$ with a few drops of $\mathrm{HNO}_{3}$ and $\mathrm{Au}$ was removed by extraction with isopropyl ether. The ether phase was adjusted to a standard volume and gold was determined by counting with a 47-cc Ge(Li) detector and a multichannel analyzer.

The aqueous phase was evaporated nearly to dryness and the residue taken up in $0.2 \mathrm{~N} \mathrm{HClO}_{4}$. Silver was separated by extraction into $\mathrm{CHCl}_{3}$ of the dibenzyldithiocarbamate. Any entrained $\mathrm{Sc}$ and rare earth activities were removed by washing the $\mathrm{CHCl}_{3}$ phase with a small amount of $0.2 \mathrm{~N} \mathrm{HClO}_{4}$. The $\mathrm{CHCl}_{3}$ phase was adjusted to a standard volume and silver was determined by counting with a 3 in $x 3$ in $N a I(T I)$ detector and a multichannel analyzer. This method gave a silver fraction of high radiochemical purity, in contrast to other methods tried, including precipitation of $\mathrm{AgCl}$ and of $\mathrm{Ag}$ metal which give products contaminated with Sc, Ta, or various rare earths.

The remaining aqueous phase, $00.2-0.5 \mathrm{~N}^{-} \mathrm{HClO}_{4}$ and containing the cobalt, had too much activity from ${ }^{46} \mathrm{Sc}$ and 
various rare earths, including ${ }^{152} \mathrm{Eu}$ to be counted directly. Therefore the bulk of the Eu and heavy rare earths were removed by extraction with HDEHP. Sc was removed by a second HDEHP extraction after acidification with $\mathrm{HNO}_{3}$. Cobalt was then determined in the aqueous phase by counting with a 47-cc $\mathrm{Ge}(\mathrm{Li})$ detector to separate the ${ }^{60} \mathrm{Co}$ peaks from those of ${ }^{122}$ Ta and remaining traces of ${ }^{152}$ Eu.

In all cases concentrations were determined by comparison with standards ( $\mathrm{CO}, \mathrm{Ag}$, and $\mathrm{Au}$ metal foils) which had been irradiated simultaneously with the samples, dissolved, and made up to the same volume for counting.

The results obtained are shown in Tables 25, 26, and 27. The scatter in the $\mathrm{Ag}$ and Co results for the $500 \mathrm{ppm}$ glass may be due in part to the presence of a small amount of precipitate, probably $\mathrm{Ta}_{2} \mathrm{O}_{5}$, which could be observed when the samples were taken up in $0.2 \mathrm{~N}^{-} \mathrm{HClO}_{4}$ following the evaporation to dryness after Au extraction. This precipitate was not present in the more dilute $50 \mathrm{ppm}$ and $1 \mathrm{ppm}$ glasses.

Table 25. Results of analysis of 1 ppm glass (SRM 615).

\begin{tabular}{|c|c|c|c|}
\hline Sample & Co, $\mathrm{ppm}$ & $\mathrm{Ag}, \mathrm{ppm}$ & $\mathrm{Au}, \mathrm{ppm}$ \\
\hline Rod \# 2A & $\begin{array}{l}0.724 \\
0.725\end{array}$ & $\begin{array}{l}0.470 \\
0.455\end{array}$ & $\begin{array}{l}0.591 \\
0.565\end{array}$ \\
\hline $36 \mathrm{~A}$ & $\begin{array}{l}0.738 \\
0.741\end{array}$ & $\begin{array}{l}0.469 \\
0.468\end{array}$ & $\begin{array}{l}0.591 \\
0.572\end{array}$ \\
\hline $40 \mathrm{~A}$ & $\begin{array}{l}0.723 \\
0.729\end{array}$ & 0.460 & $\begin{array}{l}0.557 \\
0.590\end{array}$ \\
\hline $69 \mathrm{~A}$ & $\begin{array}{l}0.709 \\
0.744\end{array}$ & $\begin{array}{l}0.460 \\
0.446\end{array}$ & $\begin{array}{l}0.547 \\
0.560\end{array}$ \\
\hline $76 \mathrm{~A}$ & $\begin{array}{l}0.730 \\
0.711\end{array}$ & & $\begin{array}{l}0.596 \\
0.591\end{array}$ \\
\hline $114 \mathrm{~A}$ & $\begin{array}{l}0.726 \\
0.729\end{array}$ & & $\begin{array}{l}0.541 \\
0.559\end{array}$ \\
\hline $\bar{x} \pm S$ & $\begin{array}{r}0.727 \\
\pm 0.011\end{array}$ & $\begin{array}{r}0.461 \\
\pm 0.008\end{array}$ & $\begin{array}{r}0.572 \\
\pm 0.019\end{array}$ \\
\hline
\end{tabular}


Table 26. Results of analysis of 50 ppm glass (SRM 613).

\begin{tabular}{|c|c|c|c|c|}
\hline \multirow{2}{*}{$\frac{\text { Sample }}{\text { Rod \#2 }}$} & \multicolumn{2}{|c|}{ Co, $\mathrm{ppm}$} & $\mathrm{Ag}, \mathrm{ppm}$ & $\mathrm{Au}, \mathrm{ppm}$ \\
\hline & 37.1 & $\begin{array}{l}36.3 \\
35.2\end{array}$ & $\begin{array}{l}25.0 \\
24.0\end{array}$ & $\begin{array}{l}5.62 \\
5.57\end{array}$ \\
\hline 37 & $\begin{array}{l}36.8 \\
35.9\end{array}$ & $\begin{array}{l}34.9 \\
34.1\end{array}$ & 21.8 & $\begin{array}{l}5.44 \\
5.41 \\
5.41\end{array}$ \\
\hline 44 & $\begin{array}{l}35.6 \\
36.1\end{array}$ & $\begin{array}{l}35.4 \\
33.7\end{array}$ & $23 \cdot 3$ & $\begin{array}{l}5.62 \\
5.38 \\
5.26\end{array}$ \\
\hline 68 & $\begin{array}{l}37.6 \\
35.6\end{array}$ & $\begin{array}{l}36.5 \\
35.0\end{array}$ & $\begin{array}{l}24.1 \\
25.2\end{array}$ & $\begin{array}{l}5.31 \\
5.30 \\
5.20\end{array}$ \\
\hline 75 & 35.1 & $\begin{array}{l}34.8 \\
35.8\end{array}$ & $\begin{array}{l}23.0 \\
22.7\end{array}$ & $\begin{array}{l}5.36 \\
5.14\end{array}$ \\
\hline 114 & 34.1 & $\begin{array}{l}35.4 \\
35.8\end{array}$ & $\begin{array}{l}21.4 \\
23.8\end{array}$ & $\begin{array}{l}5.43 \\
5.14\end{array}$ \\
\hline $\bar{x} \pm S$ & $35.5 \pm$ & & $23.4 \pm 1.2$ & \\
\hline
\end{tabular}

Table 27. Results of analysis of 500 ppm glass (SRM 61l).

Sample

Rod \# 2

13

18

48

56

66

78

106

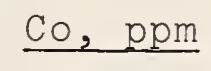

367

408

393

384

370

368

360

372
Ag, $\underline{p p m}$

221

255

226

233

225

361
Au, ppm

22.9

25.3

24.0

24.9

23.6

23.8

$\bar{x} \pm S$

$378 \pm 16$

$237 \pm 17$

$24.1 \pm 0.9$

(B. A. Thompson) 
A survey analysis was conducted on the trace elements in glass to which $20 \mathrm{ppb}$ of 61 trace elements had been added. The procedure used was that previously reported for the analysis of base glass; i.e.

1) irradiation in RT-3 for 15 minutes

2) washed with $1: 1 \mathrm{HNO}_{3}$

3) dissolution with $\mathrm{HCIO}_{4}$ and $\mathrm{HF}$

4) removal of $\mathrm{Na}-24$ with HAP column

5) 30 and 720 minute count on a $47 \mathrm{cc}$ Ge(Li) detector coupled to a 2048 channel analyzer

6) peak location and area calculation via the ALSPIS computer program written by $H$. P. Yule.

Gold, europium, copper, lanthanum, antimony, gallium, and scandium were observed in all samples. The results are given in table 28. Scandium results have a greater than

Table 28. Glass impurities.

\begin{tabular}{|c|c|c|c|c|c|c|c|}
\hline Samp Ie & $\mathrm{Au}$ & Er & $\mathrm{Cu}$ & $\underline{\mathrm{La}}$ & $\underline{S b}$ & $\underline{\mathrm{Ga}}$ & $\underline{\mathrm{Sc}}$ \\
\hline $2 \mathrm{~A}$ & 0.169 & 0.015 & 0.827 & 0.032 & 0.081 & 0.233 & 0.0 \\
\hline $2 \mathrm{~A}$ & 0.205 & 0.013 & 0.751 & 0.028 & & & \\
\hline 33 & 0.176 & 0.016 & 0.781 & 0.033 & 0.077 & 0.253 & \\
\hline 33 & 0.197 & 0.013 & 0.715 & 0.038 & & 0.2 & \\
\hline 43 & 0.181 & 0.015 & 0.808 & 0.039 & 0.080 & 0.2 & \\
\hline 43 & 0.178 & 0.016 & 0.922 & 0.035 & & 0.1 & \\
\hline $43 \mathrm{~A}$ & 0.206 & 0.016 & 0.831 & 0.035 & 0.080 & 0.2 & \\
\hline $43 \mathrm{~A}$ & 0.138 & 0.014 & 0.797 & 0.028 & & 0.25 & \\
\hline 51 & 0.180 & 0.017 & 0.858 & 0.038 & 0.076 & 0.24 & . \\
\hline 51 & 0.175 & 0.014 & 0.827 & 0.038 & & 0.1 & \\
\hline $51 \mathrm{~A}$ & 0.165 & 0.015 & 0.758 & 0.035 & 0.071 & 0.23 & \\
\hline $51 \mathrm{~A}$ & 0.7 & 0.016 & & 0.036 & 00 & & \\
\hline 16 & 0.7 & 0.016 & $0 . ?$ & 0.035 & 0.080 & 0. & \\
\hline 78 & 0. & & & 0.02 & & & \\
\hline $76 \mathrm{~A}$ & 0.1 & 0.0 & 0 . & 0.03 & 0.081 & 0.2 & \\
\hline $76 \mathrm{~A}$ & 0.189 & 0.015 & & 0.035 & & $0.2^{2}$ & \\
\hline
\end{tabular}

Recommended

Value

$$
\begin{array}{rcccccr}
0.179 & 0.015 & 0.808 & 0.035 & 0.078 & 0.231 & 0.026 \\
S \quad \pm 0.018 & \pm 0.0013 & \pm 0.049 & \pm 0.003 & \pm 0.003 & \pm 0.025 & 0.005 \\
t_{S} / \sqrt{n} \pm 0.010 & \pm 0.0007 & \pm 0.027 & \pm 0.0018 & \pm 0.003 & \pm 0.014 & 0.004
\end{array}
$$


average uncertainty due to the low solubility of scandium chloride in 8-12N HCl. Gold values have been corrected for self absorption in the standard.

(H. L. Rook and W. D. Kinard)

4. Survey of SRM One ppm Trace Elements in Glass

A survey analysis was conducted on the trace elements in glass to which 1 ppm of 61 trace elements had been added. The procedure was the same as for that of the $20 \mathrm{ppb}$ glass reported above.

Gold, europium, lanthanum, antimony, gallium, scandium, and copper were observed in all samples. The results are given in table 29. The gallium results are only significant to $\sim \pm 10 \%$ since europium in the 1 ppm glass increased by a factor of 10 relative to gallium when compared to the $20 \mathrm{ppb}$ glasses. This necessitated the use of a less abundant photopeak for analysis.

Table 29. Analytical Results for 1 ppm Glass.

Concentration (ppm)

\begin{tabular}{|c|c|c|c|c|c|c|c|}
\hline $\begin{array}{c}\text { No. } \\
\end{array}$ & $\underline{\mathrm{Au}}$ & $\underline{\mathrm{La}}$ & $\underline{\mathrm{Eu}}$ & $\underline{\mathrm{Sb}}$ & $\mathrm{Ga}$ & $\underline{\mathrm{Sc}}$ & $\underline{\mathrm{Cu}}$ \\
\hline $\begin{array}{l}2 A \\
36 A \\
40 A \\
69 A \\
76 A \\
114 A \\
76\end{array}$ & $\begin{array}{l}0.436 \\
0.438 \\
0.408 \\
0.332 \\
0.308 \\
0.383 \\
0.369\end{array}$ & $\begin{array}{l}0.842 \\
0.842 \\
0.826 \\
0.835 \\
0.810 \\
0.842 \\
0.839\end{array}$ & $\begin{array}{l}0.998 \\
1.001 \\
0.979 \\
0.979 \\
0.972 \\
1.015 \\
1.009\end{array}$ & $\begin{array}{l}1.06 \\
0.98 \\
1.09 \\
1.13 \\
1.12 \\
1.04 \\
1.05\end{array}$ & $\begin{array}{l}1.4 \\
1.5 \\
1.2 \\
1.3 \\
1.3 \\
1.2 \\
1.2\end{array}$ & $\begin{array}{l}0.565 \\
0.579 \\
0.603 \\
0.597 \\
0.592 \\
0.596 \\
0.606\end{array}$ & $\begin{array}{l}1.56 \\
1.61 \\
1.44 \\
1.65 \\
1.69 \\
1.64 \\
1.52\end{array}$ \\
\hline $\begin{array}{l}\text { Ave } \\
\text { Ts } / \sqrt{n}\end{array}$ & $\begin{array}{l}0.382 \\
0.046\end{array}$ & $\begin{array}{l}0.834 \\
0.011\end{array}$ & $\begin{array}{l}0.991 \\
0.016\end{array}$ & $\begin{array}{l}1.07 \\
0.048\end{array}$ & $\begin{array}{l}1.3 \\
0.11\end{array}$ & $\begin{array}{l}0.591 \\
0.013\end{array}$ & $\begin{array}{l}1.59 \\
0.080\end{array}$ \\
\hline
\end{tabular}


5. The Determination of Manganese in Trace Elements in Glass Samples

Manganese was determined in glass disc samples at NBS as part of the SRM certification. Manganese is one of 61 different elements which will be certified in concentrations ranging from 1 to $500 \mathrm{ppm}$.

a. Experimental

The glass discs were weighed and encapsulated in polyethylene tubing. The weights ranged from 0.40 gram to 0.80 gram. Copper foils were attached to each sample and standaro for flux normalization. Manganese metal (99.99+ purity) was the standard used. The samples and standard were irradiated at appropriate times in the NBSR pneumatic tube facility at a flux of $\sim 5 \times 10^{13} \mathrm{n} \cdot \mathrm{cm}^{-2} \mathrm{sec}^{-1}$.

The discs were washed with $1: 1$ nitric acid for 1 minute and dried with ethyl alcohol. The discs.were then dissolved with $\mathrm{HF}-\mathrm{HClO}_{4} \mathrm{l}: \mathrm{l}$ in a Teflon beaker. The solution was passed through a hydrated antimony pentoxide (HAP) column to remove the sodium-24 activity [18,8]. An additional $5 \mathrm{ml}$ of concentrated $\mathrm{HCl}$ was used to wash the column. The eluent was made $7 \mathrm{M}$ in $\mathrm{HCl}$ and extracted with isopropyl ether to remove $\mathrm{Ga}^{72}$ which has a $0.840 \mathrm{MeV}$ peak which interferes with the $0.845 \mathrm{MeV}$ peak of manganese-56. The aqueous phase was made to a constant volume and counted on a 3 in. $x 3$ in. NaI detector with 400 channel analyzer. The standards were dissolved and counted at the same geometry as the samples. In the case of the $500 \mathrm{ppm}$ glass, the additional activities made determination of the Mn difficult without further separations. In this case the sample was dissolved in hot I:I $\mathrm{HClO}_{4}-\mathrm{HF}$, taken to incipient dryness and manganese precipitated as the dioxide from $6 \mathrm{M}^{\mathrm{HNO}_{3}}$ with $\mathrm{KBrO}_{3}$. The manganese concentrations were calculated using the standard comparator method.

b. Results

The results are given in table 30 . 62 
Table 30. Manganese concentrations in trace elements in glass samples.

\begin{tabular}{|c|c|c|c|c|c|}
\hline $\begin{array}{l}\text { Sample } \\
\text { No. } \\
\end{array}$ & $\begin{array}{l}\text { Disc } \\
\text { No. }\end{array}$ & $\begin{array}{c}\text { Amount Found } \\
(\mathrm{ppm}) \\
\end{array}$ & $\begin{array}{l}\text { Sample } \\
\text { No. } \\
\end{array}$ & $\begin{array}{l}\text { Disc } \\
\text { No. }\end{array}$ & $\begin{array}{c}\text { Amount Found } \\
(\mathrm{ppm}) \\
\end{array}$ \\
\hline \multirow{8}{*}{$\begin{array}{l}\text { Base } \\
\text { glass }\end{array}$} & \multirow[t]{2}{*}{$\begin{array}{l}\text { Rod } \\
\quad 61 \mathrm{~A}\end{array}$} & \multirow{2}{*}{$\begin{array}{r}.512 \\
.519\end{array}$} & \multirow[t]{8}{*}{ SRM 611} & $\begin{array}{r}\text { Rod } \\
56\end{array}$ & 469 \\
\hline & & & & 56 & 477 \\
\hline & \multirow[t]{2}{*}{$3 I A$} & \multirow{2}{*}{$\begin{array}{l}.502 \\
.499\end{array}$} & & 48 & 466 \\
\hline & & & & 48 & 487 \\
\hline & \multirow[t]{2}{*}{$117 \mathrm{~A}$} & \multirow{2}{*}{$\begin{array}{l}.461 \\
.461\end{array}$} & & 2 & 479 \\
\hline & & & & 18 & 469 \\
\hline & \multirow[t]{2}{*}{$107 \mathrm{~A}$} & \multirow{2}{*}{$\begin{array}{l}.462 \\
.458\end{array}$} & & 66 & 484 \\
\hline & & & & 106 & 477 \\
\hline
\end{tabular}

$\begin{array}{rrr}41 \mathrm{~A} & .413 & \\ & & .417 \\ 8 \mathrm{~A} & .492 & \\ & .468 \\ 87 \mathrm{~A} & . & \\ & .518 \\ & .513 \\ & .522\end{array}$

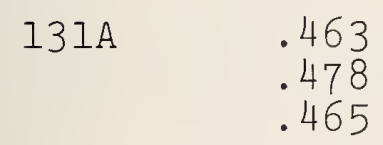

Average $\quad 470 \pm .014^{\mathrm{a}} \mathrm{ppb}$

$\overline{a_{\text {Uncertainties are }}} \mathrm{s} / \sqrt{\mathrm{n}}$ at $95 \%$ C. L. (T. E. Gills)

6. Intermediate Purity sulfur

A survey analysis was conducted on the intermediate purity sulfur with emphasis on sample homogeneity. Replicate 0.5 gram samples were taken from each batch by placing a sulfur lump in a new polyethylene bag and fracturing it with a soft blow from a plastic beaker. Enough pieces to make 0.5 gram were sealed in precleaned medical grade polyethylene tubing, irradiated in RT-3 of the NBSR for 15 minutes. The samples were transferred to clean polyvials and counted on the $47 \mathrm{cc}$ 
Table 31. Impurities found in intermediate purity sulfur (ppm by weight).

LOT - I

Sample

$\underline{M n}$

As

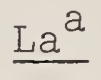

$\underline{\mathrm{Cu}^{\mathrm{b}}}$

$\underline{\mathrm{Na}}$

$\begin{array}{lllllll}I \operatorname{can} I & 0.7 \times 10^{-4} & 0.033 & 0.026 & \text { N.D. } & 0.10 \\ 1 \operatorname{can} I & 2.3 \times 10^{-4} & 0.023 & 0.015 & \text { N.D. } & 0.26\end{array}$

1 can 2

$2.4 \times 10^{--4}$

0.025

N.D.

N.D.

0.03

$2 \operatorname{can} 2$

$1.5 \times 10^{-4}$

0.030

N.D.

N.D.

0.10

$1 \operatorname{can} 3$

$6.5 \times 10^{-4}$

0.050

N.D.

N.D.

0.21

2 can 3

$6.1 \times 10^{-4}$

0.042

N.D.

N.D.

0.36

$1 \operatorname{can} 4$

$1.0 \times 10^{-3}$

0.01

0.27

0.035

0.10

$2 \operatorname{can} 4$

$1.5 \times 10^{-3}$

0.19

0.14

0.037

0.10

1 can 5

$1.5 \times 10^{-4}$

2 can 5

$1.3 \times 10^{-4}$

0.029

0.19

0.031

0.08

0.027

0.15

0.55

0.03

bimit of detection under contitions used $\sim 0.005 \mathrm{ppm}$. Limit of detection under conditions used $20.001 \mathrm{ppm}$ N.D. = not detected.

Ge(Li) detector coupled with a 2048 channel analyzer. The results are given in table 31 .

Impurities found to be present were manganese, arsenic, lanthanum, copper, and sodium. Cans 3 and 4 were found to have approximately a factor of 3 and 10 higher concentrations of trace elements respectively. The large variation in results indicated a high degree of inhomogeneity. Because of this, a second analysis was carried out where samples were segregated between pieces taken from the smooth surface, which cooled rapidly on a plastic sheet and pieces taken from the interior of the sample. The results are shown in table 32. These results clearly indicate a segregation of impuri64 
Table 32. Variation in impurities found - surface vs interior of sample (ppm by weight).

Sample
$\underline{\mathrm{Mn}}$
$\mathrm{Cu}$

As

Smooth Surface

$\begin{array}{lllll}1 & 0.7 \times 10^{-3} & 0.9 \times 10^{-2} & 4 \times 10^{-3} & 0.014 \\ 2 & 0.9 \times 10^{-3} & \text { N.D. } & \text { N.D. } & 0.015 \\ 3 & 3.1 \times 10^{-3} & 1.1 \times 10^{-2} & 3 \times 10^{-3} & 0.008 \\ 4 & 0.9 \times 10^{-3} & 0.7 \times 10^{-2} & \text { N.D. } & 0.017\end{array}$

Average $\quad 1.4 \times 10^{-3}$

$0.8 \times 10^{-2}$

$1.8 \times 10^{-3}$

0.013

$\begin{array}{lllll}1 & 5.6 \times 10^{-3} & 8 \times 10^{-2} & 1.1 \times 10^{-2} & 0.008 \\ 2 & 3.3 \times 10^{-3} & 5 \times 10^{-2} & 3.2 \times 10^{-2} & 0.019 \\ 3 & 7.0 \times 10^{-3} & 8 \times 10^{-2} & 1.2 \times 10^{-2} & 0.005 \\ 4 & 1.5 \times 10^{-3} & 2 \times 10^{-2} & \text { N.D. } & 0.010\end{array}$

Average $\quad 4.4 \times 10^{-3}$

$5.7 \times 10^{-2}$

$1.5 \times 10^{-2}$

0.011

N.D. = not detected.

ties, except arsenic, to the interior of the sulfur cake. This may have been due to an internal "zone refining" effect as the sulfur cooled.

(H. L. Rook)

7. Potassium Chloride Single Crystal

A survey analysis was conducted of a high purity KCl crystal to determine if impurities from its plastic container were contaminating the crystal surface. Two different procedures were used to detect surface contamination. First, a KCl crystal was transferred and sealed into medical grade polyethylene which had been washed in l:l nitric acid, rinsed with deionized water, followed by absolute ethanol and drying. The sample trnasfer from package to polyethylene tubing was 
conducted without contact with any foreign implements. The sample was irradiated in RT-4 for 5 minutes. Following irradiation, the sample was etched in 3 successive portions of deionized water, each removing about $50 \mathrm{mg}$ of $\mathrm{KCl}$. The solutions were counted in a standard geometry on the $47 \mathrm{cc}$ $\mathrm{Ge}(\mathrm{L}$ ) detector for 30 minutes after a 4 hour delay to allow the decay of chlorine-38. All solutions contained only activity derived from potassium and bromine. The weight percent bromine was the same in all three fractions thus it was concluded to be a bulk impurity rather than a surface contamination.

The second and more sensitive procedure was to open a package with a scalpel along one edge, remove the KCl crystal and reseal the package with polyester tape. The package was irradiated in RT-3 for 25 minutes, the tape removed, the $\mathrm{KCl}$ crystal reinserted, vigorously rubbed with the inside of the package, removed and counted. The only contamination found other than potassium and chlorine, was sodium in the level of $\sim 10^{-3} \mathrm{\mu g}$. It was felt from these results that no significant contamination of the $\mathrm{KCl}$ crystal was occurring from contact with the packaging material.

(H. L. Rook)

8. Determination of Indium and Copper in SRM High Purity Gold Wire by Neutron Activation Analysis

Samples of high purity gold wire were received from the Office of Standard Reference Materials for analysis of impurities. Information available about the samples, from resistivity measurements and survey analyses, indicated that the two trace elements indium and copper were present in the gold at sub-ppm levels. If possible, activation analysis was to be used to obtain a quantitative measurement of these impurity levels for certification.

The gold matrix presented a formidable problem in this analysis. Gold, with a 98.8 barn cross-section for thermal 
neutrons and a $64.8 \mathrm{~h}$ half-life produced approximately 20 millicuries of ${ }^{198} \mathrm{Au}$ for a $50 \mathrm{mg}$ sample irradiated for $30 \mathrm{~m}$ at a thermal neutron flux of $8 \times 10^{12} \mathrm{n} \cdot \mathrm{cm}^{-2} \mathrm{sec}^{-1}$. In contrast, a copper impurity of $0.1 \mathrm{ppm}$ would produce only $5 \times 10^{-7}$ as much radioactivity as the gold. In addition, both the indium-116m $\left(\mathrm{T}_{1 / 2}=54 \mathrm{~m}\right)$ and the copper-64 $\left(\mathrm{T}_{1 / 2}=12.9 \mathrm{~h}\right)$ have considerably shorter half-lives than the gold. As a result of these unfavorable analysis conditions, a radiochemical separation of the copper and indium was required. This separation not only had to be rapid, in order to count the short-lived indium radioisotope, but had to be very clean, with a decontamination factor of at least $10^{5}$ and preferably $>10^{6}$

A radiochemical separation which would fill these requirements was developed. It consisted of spontaneous deposition of the gold matrix on a metallic silver-asbestos column, with a final purification by precipitation of the copper and indium cyanoferrates.

a. Experimental

The samples as received were in the form of $1.4 \mathrm{~mm}$ diameter wire. They were separated into short sections and flattened between two clean lucite blocks to reduce neutron self-shielding effects. Individual samples weighed approximately $50 \mathrm{mg}$ and were irradiated along with suitable standards and flux monitors for 30 minutes in the NRL in-core facility at a thermal neutron flux of $8 \times 10^{12} \mathrm{n} \cdot \mathrm{cm}^{-2} \mathrm{sec}^{-1}$. After irradiation, the samples and standards were returned to NBS, etched in aqua regia to remove exterior contamination, and then dissolved for the analysis with addition of copper and indium carriers. After dissolution, perchloric acid was added and the solutions taken to fumes of perchloric acid to remove the chloride ions. The silver column was prepared by adding a metallic silver slurry to purified asbestos and thoroughly mixing in a blender. This suspension of silver metal on asbestos was then poured into a filter chimney and 
kept under water. After preparation of the column, the concentrated perchloric acid sample solution was diluted and passed twice through the silver-asbestos column. The column was then washed once with dilute perchloric acid. This procedure provided both a $10^{6}$ decontamination of the filtrate from gold-198 and a quantitative recovery of the copper and indium in the eluate.

In a final separation step, to remove the last traces of gold and to remove a small amount of sodium-24 contamination, the copper and indium were precipitated as their cyanoferrates. To do this, the eluate was $\mathrm{pH}$ adjusted and a saturated solution of $\mathrm{K}_{4} \mathrm{Fe}(\mathrm{CN})_{6}$ added. The highly insoluble copper and indium cyanoferrates were centrifuged, the supernate decanted, and the precipitates then washed with dilute $\mathrm{K}_{4} \mathrm{Fe}(\mathrm{CN})_{6}$ solution.

The copper and indium precipitates were counted while still in the centrifuge tubes, on a $3 \times 3$ NaI(TI) scintillation detector with a 400 channel PHA. Half-lives of both copper and indium were measured to verify isotopic purity, and appropriate calculations and corrections applied to the data.

b. Results and Discussion

The results of these analyses are found in table 33 . These results include a calculated neutron self-shielding correction of almost $20 \%$.

The analytical results have rather large uncertainties ( $\pm 22 \%$ for copper and $\pm 26 \%$ for indium) due primarily to the extremely unfavorable matrix involved. In spite of the difficulties encountered, the agreement between these values and other analytical techniques was satisfactory and the high-purity gold was certified for its copper and indium impurity concentrations [20]. 
Table 33. Analyses for copper and indium in SRM gold wire.

\section{Sample}

1

2

3

4

Mean

ts $/ \sqrt{n}$ b
Element Determine

\section{Copper}

$0.12,0.11$

0.25

N.D. ${ }^{\mathrm{a}}$

$0.11,0.21,0.15$

$0.18,0.16$

$$
0.16
$$

0.010

$\pm 0.034$

0.0027

$a_{\text {Not determined. }}$.

b5\% confidence level.

(W. D. Kinard, D. A. Becker, and P. D. LaFleur)

9. The Determination of Cobalt in Cobalt-Aluminum Flux Wires

The NBS Standard Reference Material program is certifying cobalt-aluminum wires for use in neutron flux determinations in the nuclear industry. The wires are approximately $10 \mathrm{mil}$ diameter and contain $0.1 \%$ cobalt. These wires were analyzed by the Activation Analysis Section for their homogeneity and cobalt content.

a. Experimental

The wires were cut into 1.5 inch lengths and weighed. The average weight of each sample was approximately $14 \mathrm{mg}$. The wires were then encapsulated in clean quartz. Attached to each sample was a $5.0 \mathrm{mg}$ copper foil to monitor the flux variation. Cobalt metal $(99.9+\%)$ was also encapsulated in 
Table 34. Analysis results for cobalt in cobalt aluminum wire.

\begin{tabular}{|c|c|c|c|c|c|}
\hline Sample No. & $\begin{array}{c}\text { Cobalt } \\
\% \\
\end{array}$ & $\begin{array}{l}\text { Average \% } \\
\text { Cobalt } \\
\end{array}$ & Sample No. & $\begin{array}{c}\text { Cobalt } \\
\% \\
\end{array}$ & $\begin{array}{c}\text { Average \% } \\
\text { Cobalt }\end{array}$ \\
\hline 1 & $\begin{array}{l}.118 \\
.117 \\
.115\end{array}$ & $.117 \pm .004$ & 9 & $\begin{array}{l}.117 \\
.118 \\
.118\end{array}$ & $.118 \pm .001$ \\
\hline 2 & $\begin{array}{l}.117 \\
.118 \\
.116\end{array}$ & $.117 \pm .002$ & 10 & $\begin{array}{l}.116 \\
.117 \\
.119\end{array}$ & $.117 \pm .004$ \\
\hline 3 & $\begin{array}{l}.118 \\
.122 \\
.119\end{array}$ & $.120 \pm .05$ & 11 & $\begin{array}{l}.118 \\
.114 \\
.116\end{array}$ & $.116 \pm .005$ \\
\hline 4 & $\begin{array}{l}.117 \\
.117 \\
.116\end{array}$ & $.117 \pm .001$ & 12 & $\begin{array}{l}.119 \\
.118 \\
.119\end{array}$ & $.119 \pm .001$ \\
\hline 5 & $\begin{array}{l}.117 \\
.118 \\
.118\end{array}$ & $.117 \pm .002$ & 13 & $\begin{array}{l}.116 \\
.119 \\
.115\end{array}$ & $.117 \pm .005$ \\
\hline 6 & $\begin{array}{l}.116 \\
.117 \\
.118\end{array}$ & $.117 \pm .002$ & 14 & $\begin{array}{l}.118 \\
.115 \\
.116\end{array}$ & $.116 \pm .004$ \\
\hline 7 & $\begin{array}{l}.119 \\
.121 \\
.116\end{array}$ & $.119 \pm .006$ & 15 & $\begin{array}{l}.110 \\
.114 \\
.109\end{array}$ & $.111 \pm .006$ \\
\hline 8 & $\begin{array}{l}.117 \\
.118\end{array}$ & $.117 \pm .006$ & & & \\
\hline
\end{tabular}

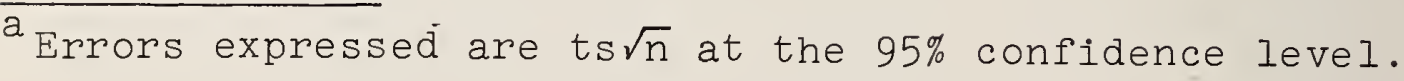

quartz and was irradiated along with the samples. The samples and standards were irradiated for approximately five hours at a neutron flux of $8 \times 10^{12} \mathrm{n} \cdot \mathrm{cm}^{-2} \mathrm{sec}^{-1}$ in the glory tube of the Naval Research Reactor in Washington, D. C.

After irradiation the samples were allowed to stand for three weeks so that the sodium-24 activity produced by the ${ }^{27} \mathrm{AI}(n, \alpha)^{24} \mathrm{Na}$ reaction on the matrix could decay. Sodium-24 
has a fifteen hour half-lif'e and a $1.36 \mathrm{MeV}$ gamma ray that is very close to the $1.33 \mathrm{MeV}$ of $\mathrm{Co}^{60}$. After the sodium-24 activity had decayed, the samples and standards were counted using a 3 in. $x 3$ in. NaI(Tl) scintillation detector and a 400 channel pulse height analyzer. The cobalt content of the samples were calculated using the standard-comparator method. The results were normalized for flux variations using the flux monitors. The results are given in table 34 .

b. Results and Discussion

The results showed no significant difference between samples 1-14. Sample 15 is significantly lower in cobalt content. This result was confirmed by spectrophotometry.

(T. E. Gills and D. A. Becker)

10. Analysis of Cast Iron Steels for Tellurium

SRM No. 1140 (Cast Iron Steel) was analyzed for its tellurium content using destructive neutron activation analysis $[21,22]$. The procedure utilizes the nuclear reaction:

$$
\mathrm{Te}^{130}(\mathrm{n}, \mathrm{\gamma}) \mathrm{Te}^{131} \mathrm{\beta}^{\prime} \mathrm{I}^{131}
$$

a. Experimental

\section{$25 \mathrm{~m}$}

Individual cast iron samples of $\sim 145 \mathrm{mg}$ were weighed and encapsulated in polyethylene tubing. To each sample was attached a copper flux monitor to measure flux variation. The samples were irradiated for $\sim 1$ hr at a flux of $\sim 5 \times 10^{13}$ $\mathrm{n} . \mathrm{cm}^{-2} \mathrm{sec}^{-1}$. After allowing to decay for 12 hours, the iron samples were dissolved in a closed system and the radioactive iodine distilled and precipitated as AgI. Iodine-125 was used as a tracer for yield measurements. Standards were small ( $\sim \mathrm{mg}$ ) particles of 5-9's tellurium metal.

b. Results

The results are shown in table 35. The cause of the lack of precision is unknown at present, but may be due 
Table 35. Results of tellurium analysis in SRM cast iron steel.

$\begin{array}{lcc}\text { SRM No. } & \text { Te Concentrations } \% & \text { Average } \\ 1143 & .022 & .020 \\ & .019 & .016 \\ 1143 a & .016 & \\ & .017 & .019 \\ 1144 & .022 & \\ 1144 a & .017 & .022 \\ & .017 & \\ 1147 & .026 & .015 \\ & .011 & \\ 1147 a & .013 & .032 \\ 1148 & .029 & \\ & .036 & .015 \\ 1149 & .018 & .011 \\ & .011 & \end{array}$

either to inhomogeneity in the samples or to factors not defined in the analytical technique.

(T. E. Gills and D. A. Becker)

11. Activation Analysis of SRM Steels Using Group Radiochemical Separations

Concentrations of several minor constituents in SRM steels 1263, 1264 and 1265 have been determined by activation analysis using the NBS Reactor and group radiochemical separations reported previously [23]. For SRMs 1263 and 1264 the analyses were for acceptance testing of the sample submitted by the manufacturer, and for 1265 the purpose was certification. The results are shown in table 36 . 
Table 36. Results of analysis of SRMs 1263, 1264, 1265 (\% by weight unless otherwise noted).
Element
$\underline{1263}$
$\underline{1264}$

$\mathrm{Cu}$

As

Co

$\mathrm{Cr}$

W

$\mathrm{Sb}$,

As

La

$\mathrm{Ta}$

Al
0.0093

0.0279

0.00153

0.00052

0.00103
0.0299

0.000795 $\frac{1265(365)}{0.00593 \pm 0.00029^{a}}$

$0.000224 \pm 0.000010$

$0.00742 \pm 0.00038$

$0.00698 \pm 0.00063$

$0.000033 \pm 0.000007$

$<0.5 \mathrm{ppm}$

0.0000965

$<0.02 \mathrm{ppm}$

$<0.1 \mathrm{ppm}$

$<0.5 \mathrm{ppm}$

aimits quoted are $t s / \sqrt{n}$ for the $95 \%$ C.L.; $n=6$.

SRM ductile irons $1140,1141,1142,1140 \mathrm{~A}, 1141 \mathrm{~A}$, and Il42A were analyzed for $\mathrm{Cu}, \mathrm{As}$, and Mo by neutron activation. $\mathrm{Cu}$ and $\mathrm{As}$ were determined by counting with a $47 \mathrm{cc}$ Ge(Li) detector after extracting $\mathrm{Fe}$ and $\mathrm{Sb}$ as the chlorides with isopropyl ether. Mo was determined by a double extraction with HDEHP, using $99 \mathrm{~m}_{\mathrm{TC}}$ as a measure of the Mo present [24]. The results obtained are shown in table 37.

Table 37. Results of analysis of SRM ductile irons (\% by weight).

SRM No.

$\underline{\mathrm{Cu}}$

$\underline{\text { As }}$

Mo

1140

$0.1084 \pm 0.0018^{a}$

$0.0718 \pm 0.0022$

$0.1003 \pm 0.0021$

1141

$0.2473 \pm 0.0065$

$0.0360 \pm 0.009$

$0.0478 \pm 0.0028$

1142

$1.169 \pm 0.016$

$0.00503 \pm 0.00010$

$0.0213 \pm 0.0006$

$1140 \mathrm{~A}$

$0.1013 \pm 0.0019$

$0.0782 \pm 0.0008$

$0.1620 \pm 0.0048^{b}$

$1141 \mathrm{~A}$

$0.2331 \pm 0.0082$

$0.0213 \pm 0.0008$

$0.0439 \pm 0.0053^{b}$

$1142 \mathrm{~A}$

$1.187 \pm 0.011$

$0.00456 \pm 0.00022$

$0.0235 \pm 0.0012^{b}$

$\overline{\mathrm{a}_{\mathrm{a}} \text { Limits quoted }}$ are $t \mathrm{~s} / \sqrt{\mathrm{n}}$ for the $95 \%$ C. L.; $\mathrm{n}=6$. $n=3$. 
Molybdenum was determined in SRM lolf using the HDEHP double extraction method. The results obtained on two different samples of $101 \mathrm{f}$ were:

$$
\begin{aligned}
& 101 f-1 \quad 0.00703 \pm 0.00031 \% \text { Mo } \\
& 101 f-4 \quad 0.00668 \pm 0.00021 \% \text { Mo }
\end{aligned}
$$

where the limits are $t s / \sqrt{n}$ for the $95 \%$ C. L.; $n=6$.

Arsenic was determined in SRM $122 e$ (cast iron car wheel) by neutron activation analysis. Any potential antimony interference was removed by extraction with isopropyl ether from $\sim 7 \mathrm{~N} \mathrm{HCl}$ in the presence of $\mathrm{NaBrO}_{3}$. The value found was

$$
0.01766 \pm 0.00010 \% \text { As }
$$

where the limits are $t s / \sqrt{n}$ for the $95 \%$ C. L.; $n=6$.

(B. A. Thompson)

12. The Determination of Cobalt in SRM Steels by Neutron Activation Analysis

Cobalt was determined in NBS steels $101 f_{1}$ and $101 f_{4}$ by nondestructive neutron activation analysis. The steels are part of the certification program at the National Bureau of Standards.

a. Experimental: The steel samples were weighed ( $100 \mathrm{mg}$ ea) and encapsulated in polyethylene tubing. Copper foils were attached to both samples and standards to monitor flux variation. The samples were irraditaed for 10 minutes at a neutron flux of $1.3 \times 10^{13} \mathrm{n} \cdot \mathrm{cm}^{-2} \mathrm{sec}^{-1}$. The samples were allowed to decay for 48 hours before counting. The counting was done on a $47 \mathrm{cc}$ Ge(Li) detector in conjunction with a 4096 channel analyzer. The flux monitors were used to normalize results.

b. Results

The results of the analysis are given in table 38 . NBS certified SRM Steel No. 1166 was analyzed concurrently to verify the accuracy of analysis. 
Table 38. Results of analysis for cobalt

(\% by weight).

\begin{tabular}{lll} 
Sample & Amount found & Average \\
\cline { 2 - 2 } $\mathrm{fl}_{1}$ & .0873 & \\
& .0851 & \\
& .0869 & \\
& .0877 & \\
& .0871 & \\
$101 \mathrm{f}_{4}$ & $.0868 \pm .0012$ \\
& .0875 & \\
& .0840 & \\
& .0877 & \\
& .0853 & \\
& .0883 &
\end{tabular}

(T. E. Gills)

13. Determination of Copper and Cobalt in SRM Steel $1265 \mathrm{~B}-1$

SRM Steel No. 1265 B-l was analyzed for copper and cobalt using destructive neutron activation analysis. The sample contained as many as 13 elements which when irradiated in a thermal neutron flux produce a gamma spectrum too complex for resolution by a Ge(Li) detector. Therefore, a separation of copper and cobalt from the matrix was necessary.

a. Procedure

The steel chip samples were weighed, ( $100 \mathrm{mg}$ ea.) encapsulated in polyethylene tubing and irradiated for 10 minutes. To each sample $10 \mathrm{mg}$ copper foils were attached as flux monitors. The samples were allowed to decay for 24 hours to reduce ${ }^{56} \mathrm{Mn}$ activity. The samples were then dissolved in $\mathrm{HClO}_{4}-\mathrm{HNO}_{3}$ and ${ }^{64} \mathrm{Cu}$ and ${ }^{60} \mathrm{Co}$ were separated using a solvent extraction procedure [25]. The separated fractions were counted on a 47cc Ge(Li) detector in conjunction with a 4096 channel analyzer. Concentrations were determined using the standard comparator method. Flux monitor foils were used to normalize results.

b. Results

The results are given in table 39. 
Table 39. Results of activation analysis of SRM 1265 B-l.

$\begin{array}{ll}\text { Element } & \text { \% Found } \\ \text { Copper } & .00597 \\ & .00605 \\ & .00632 \\ & .00628 \\ & .00603 \\ & .00602 \\ & .00584 \\ & .00637 \\ & .00614 \\ & .00724 \\ & .00715 \\ & .00771 \\ & .00740 \\ & .00758 \\ & .00758 \\ & .00735 \\ & .00749\end{array}$

$\begin{array}{r}\text { Average } \pm \text { ts } / \sqrt{n} \\ (95 \% \text { C.L. }) \\ \hline\end{array}$

$.00611 \pm .00014$

$.00747 \pm .00020$

(T. E. Gills)

14. Nondestructive Determination of Manganese and Vanadium in Iron and Steel Standard Reference Materials

Nine iron-based SRM's were analyzed for their manganese and vanadium content. The method used was nondestructive neutron activation analysis. Besides the two elements of interest, the SRM had additional added elements to a maximum of 13 per SRM, averaging about 10 certified elements. The elements range in concentration from $\leq .1 \%$ to over $3 \%$ (except for major constituents such as chromium which is $220 \%$ ).

Similar samples have been priviously analyzed for manganese and vanadium in this laboratory [26, 27]. In most cases the procedures were essentially the same. Therefore only the general procedure will be discussed here.

a. Experimental

Samples were analyzed as received. Approximately 100 mgs each of the steels were sealed in polyethylene snap-cap vials. The standards were prepared by pipetting solutions of manganese and vanadium onto filter paper inside the vials 
and allowing-t dry. The vials were then heat-sealed. Copper foils were attached to both sample and standard to normalize the results.

The procedure used was as follows:

(1) The sample or standard was irradiated for $3 \mathrm{sec}$ in pneumatic tube RT-4 of the NBS reactor.

(2) Allowed the sample to decay for 3 minutes. During this time the flux monitor was removed and the sample positioned on the detector.

(3) At the end of the decay, the sample was counted for 4.0 minutes live time with the clock time recorded and used for decay correction.

The peak areas of both manganese and vanadium was integrated using Covell's method [28]. The concentrations of each sample was then calculated using the standard-comparator method. Flux variations from sample to sample were corrected using the flux monitors. Shelf-shielding and gamma-ray attenuation corrections were calculated and found to be negligible for these conditions.

b. Results and Discussion

The analysis of the eleven SRMs can be found in table 40 .

Table 40. Results of manganese and vanadium analyses of iron and steel Standard Reference Materials.

\begin{tabular}{|c|c|c|c|c|}
\hline $\begin{array}{c}\text { SRM } \\
\text { Sample }\end{array}$ & $\begin{array}{r}\text { No. Of } \\
\text { Samples }\end{array}$ & $\begin{array}{l}\text { Average \% } \\
\text { Mn Found }\end{array}$ & $\begin{array}{r}\text { No. of } \\
\text { Samples } \\
\end{array}$ & $\begin{array}{l}\text { Average } \% \\
\mathrm{~V} \text { Found }\end{array}$ \\
\hline $\begin{array}{l}101 \mathrm{fl} \\
101 \mathrm{f} 4 \\
342 \mathrm{a} \\
1261 \\
361 \\
1265 \mathrm{~B}-1 \\
1265 \\
1138 \\
1139 \\
362 \\
1262\end{array}$ & $\begin{array}{r}6 \\
6 \\
- \\
12 \\
12 \\
43 \\
8 \\
4 \\
4 \\
6 \\
6\end{array}$ & $\begin{array}{r}.0904 \pm .0013^{a} \\
.0905 \pm .0026 \\
- \\
.6845 \pm .0101 \\
.6803 \pm .0054 \\
.0066 \pm .0002 \\
.0074 \pm .0002 \\
.4393 \pm .0140 \\
.9662 \pm .0184 \\
1.024 \pm .012 \\
1.030 \pm .009\end{array}$ & $\begin{array}{l}6 \\
6 \\
5 \\
6 \\
6 \\
9 \\
9 \\
4 \\
4 \\
6 \\
6\end{array}$ & $\begin{array}{l}.0284 \pm .0003 \\
.0287 \pm .0007 \\
.0192 \pm .0009 \\
.01132 \pm .00021 \\
.01132 \pm .00028 \\
.00058 \pm .00002 \\
.00054 \pm .00002 \\
.0225 \pm .0020 \\
.2405 \pm .0081 \\
.0394 \pm .0004 \\
.0392 \pm .0007\end{array}$ \\
\hline
\end{tabular}

$a_{\text {Uncertainties are }}$ s $/ \sqrt{n}$ at $95 \%$ C. L. 
15. The Multielement Analysis of SRM Steels 1261 and and 361 by Destructive Neutron Activation Analysis

As a part of the SRM program at NBS, steel SRM's 1261 and 361 were analyzed for final certification. These steels contain approximately 13 elements of which 8 were analyzed by neutron activation analysis (W, Cr, Cu, Cg, As, Sb, La and Mo).

\section{a. Experimental}

Six samples of each of the steels were irradiated for one hour in RT-3 of the NBS Reactor. The samples were allowed to decay over night to reduce the ${ }^{56} \mathrm{Mn}$ activity. The samples were then dissolved and separated into three groups using a solvent extraction procedure [25]. W and Mo were determined by counting the $\alpha$-benzoinoxime $\mathrm{CHCl}_{3}$ fraction. T_e other elements were determined by counting the ether extraction and aqueous fraction. The counting was done on a 47 Li(Ge) detector in conjunction with a4096 pulse height analyzer.

b. Results

The results obtained are shown in table 41.

Table 41. Results of multielement analysis of SRM steels 1261 and 361 .

Element/Sample

Cobalt

Copper

Chromium

Arsenic

Antimony

Lanthanum

Molybdenum

Tungsten
1261

$.0325 \pm .0007^{a}$

$.0432 \pm .0016$

$.624 \pm .060^{\mathrm{b}}$

$.0128 \pm .0009$

$.00270 \pm .0002$

$.000378 \pm .000030$

$.1531 \pm .0180$

$.0107 \pm .0021$ $\underline{361}$

$.0329 \pm .0016^{a}$

$.0433 \pm .0004$

$.637 \pm .017^{\mathrm{b}}$

$.0145 \pm .0008$

$.00259 \pm .0002$

$.00109 \pm .00008$

$.1582 \pm .0045$

$.0107 \pm .0009$

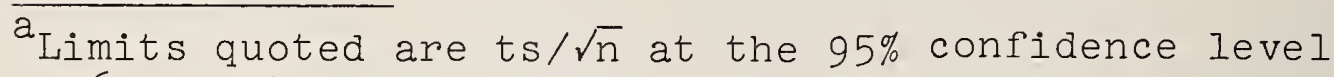
$n=6$ except where noted. $\mathrm{b}_{\mathrm{n}}=4$ 
E. Special Analyses

1. Determination of Copper Homogeneity in a Copper-Lead Alloy

In an attempt by the Activation Analysis Section to obtain an ideal flux monitor (see also section 3-C-4, this report) a bid was submitted by a private company to fabricate a homogeneous alloy of $0.01 \%$ copper in lead. The specifications stated that the absolute copper concentration was not critical, but that the homogeneity must be at least $\pm 1 / 2 \%$. The lead diluant was to be at least $99.999 \%$ pure, and samples of the lead to be used were to be submitted to us for analysis before alloying with copper.

The successful production of a homogeneous copper-lead alloy on a small scale had already been made in this laboratory [29]. In this previous work 0.1\% copper foils with an $\mathrm{S}_{\mathrm{d}}$ of $1.08 \%(\mathrm{n}=26)$ were produced. It was expected that with professional facilities a more homogeneous alloy could be produced.

a. Experimental

The foil was obtained as a series of long narrow sheets of $\sim 5$ mil thickness. After cleaning the foil surface with ethyl alcohol, samples for analysis were taken from various portions of the sheets. These samples were irradiated along with a copper standard for 15 minutes in the RT-3 pneumatic facility of the NBSR. Flux normalization was made for both samples and standards.

After verifying the radiochemical purity of the ${ }^{64} \mathrm{Cu}$ activity using a Ge(Li) detector and 4096 channel PHA, the samples and standards were counted on a 3 x $3 \mathrm{NaI}(\mathrm{TI})$ detector and 400 channel PHA.

b. Results and Discussion

The results of these analyses are much too numerous to list. However, at no time did the foils received meet specifications. In a number of cases, the homogeneity from one 
area would be quite good ( $0.5 \% \mathrm{~S}_{\mathrm{d}}$ ); however, over the whole batch the foil was quite inhomogeneous (up to $3.0 \% \mathrm{~s}_{\mathrm{d}}$ ). Reruns of our in-house produced foil showed significantly better homogeneity than the commercially produced foil.

In addition to the problem of the copper inhomogeneity, reworking of the alloy by the company introduced a relatively large, inhomogeneous manganese impurity into the foil. This impurity ranged from 28 to $24 \mathrm{ppm}$, and immediately after irradiation produced a greater radioactivity due to ${ }^{56} \mathrm{Mn}$ than that of the ${ }^{64} \mathrm{Cu}$. Thus, the alloy was determined to be unsatisfactory for use as a flux monitor material.

(W. D. Kinard and D. A. Becker)

2. Analysis of Neodymium Laser Glass by Neutron Activation

The Activation Analysis Section was asked by the Inorganic Materials Division to carry out analyses on five different neodymium laser glasses as part of an overall program of characterization of these glasses. The glasses are very complex mixtures with, in some cases, as many as ten principal constituents ( $\mathrm{Si}, \mathrm{Na}, \mathrm{Ba}, \mathrm{K}, \mathrm{Al}, \mathrm{Nd}, \mathrm{Sb}, \mathrm{Zn}, \mathrm{Li}, \mathrm{Ti}$ ). For activation analysis the constituents representing the most serious interferences to the measurement of other activities are sodium, antimony ( $1 \%$ or more in 4 of the glasses), and neodymium ( $3-5 \%$ in all 5 glasses). Neodymium is especially aggravating since on irradiation it yields three radionuclides: ${ }^{15} 1_{\mathrm{Nd}}$, with a 12 minute half-life; ${ }^{149} \mathrm{Nd}$, with a 1.8 hour half-life; and $147 \mathrm{Nd}$ with a 11 day half-life. ${ }^{151_{\mathrm{Nd}} \text { and }}$ ${ }^{149} \mathrm{Nd}$ also decay to form radioisotopes of $\mathrm{p}$ omethium with half-lives of 28 hours and 53 hours. All th se nuclides have very complex decay schemes and emit many gamm -rays.

To try to obtain the maximum amount of inf rmation with a reasonable expenditure of effort, three sets of irradiations were carried out. For each of the glasses a small sample (10-50mg) was irradiated for 2 seconds and used to determine 
$\mathrm{Na}, \mathrm{K}$, and $\mathrm{Sb}$. A larger sample (100-200 mg) of each glass was irradiated for 1 minute and dissolved in $\mathrm{HF}-\mathrm{HClO}_{4}$. After fuming to eliminate $\mathrm{HF}$, the residue was taken up in $9 \underline{\mathrm{N}} \mathrm{HCl}$ and most of the antimony was removed by extraction into isopropyl ether. Sodium was then removed by passing the aqueous phase through column of hydrated antimony pentoxide. The effluent was examined for low-level constituents of intermediate half-life using a 47-cc Ge(Li) detector. Those observed included La, Pr, Eu, and Sm. Common impurities such as $\mathrm{Cu}$ and $\mathrm{Ga}$ were not observed.

A third set of samples (100-200 mg) was irradiated for 15 minutes and set aside for two weeks to allow all shorterlives activities to decay away. After dissolution and extraction of antimony as before, the aqueous phase was counted and $\mathrm{Nd}, \mathrm{Ce}$, and $\mathrm{Ba}$ were determined. (Glass "D" did not require these chemical separations since it had very low concentrations of $\mathrm{Na}$ and $\mathrm{Sb}$. )

All the samples were irradiated in pneumatic tube RT-3 of the NBSR at a flux of about $5 \times 10^{13} \mathrm{n} \cdot \mathrm{cm}^{-2}$ sec. ${ }^{-1}$. The maximum irradiation time-sample size combination was fixed by the ${ }^{24} \mathrm{Na},{ }^{122} \mathrm{Sb}-{ }^{124} \mathrm{Sb}$, and $147 \mathrm{Nd}$ activities produced. All the samples were specially cut l-mm thick slices. With samples this thin, neutron self-shielding was not a problem even for the "D" glass with $14 \% \mathrm{Li}_{2} \mathrm{O}$. For this latter glass irradiation time was not limited by $\mathrm{Na}$ or $\mathrm{Sb}$ because these were quite low, but by $\mathrm{Nd}$ and by heating of the sample during irradiation by absorption of tritons and alpha particles from the reaction ${ }^{6} \mathrm{Li}(\mathrm{n}, \alpha)^{3} \mathrm{H}$. All the samples were rinsed in acetone and in dilute $\mathrm{HNO}_{3}$ after irradiation to remove surface contamination. Concentrations were determined by comparison to standards irradiated under the same conditions.

The results obtained are shown in Table 42. For most elements the standard deviation is estimated to be about $\pm 5 \%$. For the lower reported values of $\mathrm{Sm}$ there is severe interference from Nd and these values are probably no better than 
Table 42. Results of activation analysis of neodymium glasses.

\begin{tabular}{|c|c|c|c|c|c|}
\hline & "A" & "B" & "C" & "D" & "E" \\
\hline $\mathrm{Na}_{2} \mathrm{O}, \%$ & 7.83 & 3.19 & 6.39 & 0.044 & 6.85 \\
\hline $\mathrm{K}_{2} \mathrm{O}, \%$ & 13.8 & 16.6 & 17.1 & $<0.04$ & 9.21 \\
\hline $\mathrm{Sb}_{2} \mathrm{O}_{3}, \%$ & 0.86 & 2.54 & 0.93 & 0.0015 & 0.90 \\
\hline $\mathrm{Na}_{2} \mathrm{O}_{3}, \%$ & 5.84 & 3.53 & 5.73 & $3 \cdot 52$ & 5.52 \\
\hline $\mathrm{BaO}, \%$ & 3.27 & 3.46 & 2.83 & $<0.3$ & 5.06 \\
\hline $\mathrm{CeO}_{2}, \%$ & 0.005 & $<0.006$ & $<0.006$ & 0.52 & $<0.006$ \\
\hline $\mathrm{La}_{2} \mathrm{O}_{3}, \mathrm{ppm}$ & 10.2 & 17.8 & 7.8 & $<0.8$ & $<0.9$ \\
\hline $\operatorname{Pr}_{2} \mathrm{O}_{3}, \mathrm{ppm}$ & $<3$ & $<3$ & 8.4 & $<2$ & $<3$ \\
\hline $\mathrm{Eu}_{2} \mathrm{O}_{3}, \mathrm{ppm}$ & 0.6 & $\leq 0.1$ & 1.1 & $<0.5$ & 29.7 \\
\hline $\mathrm{Sm}_{2} \mathrm{O}_{3}, \mathrm{ppm}$ & 0.2 & 0.2 & 2.2 & $<0.03$ & 0.37 \\
\hline
\end{tabular}

$\pm 20 \%$. For the lower reported values of Eu and Ce the signalto-background ratio was poor and these values also are probably no better than $\pm 20 \%$.

(B. A. Thompson)

3. Determination of Trace Elements in Ruby Laser Crystals

At the request of the Inorganic Materials Division a number of ruby laser crystals were analyzed quantitatively for trace elements by neutron activation. The analysis is quite difficult for other methods such as emission spectroscopy and spark source mass spectrometry because the ruby $\left(\alpha-\mathrm{Al}_{2} \mathrm{O}_{3}\right.$ with $\left.\leq 0.1 \% \mathrm{Cr}_{2} \mathrm{O}_{3}\right)$ is such a good insulator and thus does not spark. $\mathrm{Al}_{2} \mathrm{O}_{3}$ is almost an ideal matrix for activation analysis since the longest lived radionuclide produced from the matrix is $15-$ hour ${ }^{24} \mathrm{Na}$ formed by the reaction $27 \mathrm{Al}(\mathrm{n}, \alpha)^{24} \mathrm{Na}$. 
$\alpha-\mathrm{Al}_{2} \mathrm{O}_{3}$ is extremely refractory and so all the analyses were carried out nondestructively. The crystals were cut to a standard geometry (see fig. 22) before irradiation and were irradiated in pneumatic tube RT-4 of the NBSR which has a high ratio of thermal-to-fast neutrons. This facility

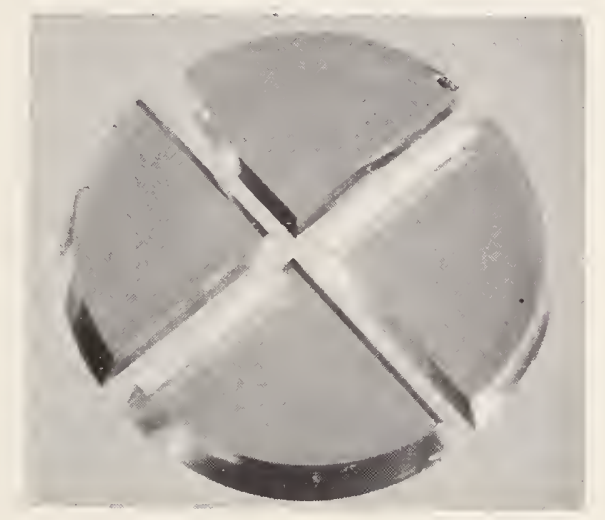

Figure 22. Ruby crystal as cut for analysis.

has a flux of $\sim 10^{13} \mathrm{n} \cdot \mathrm{cm}^{-2}$ sec. ${ }^{-1}$. After irradiation and decay of ${ }^{28} \mathrm{Al}$, the samples were counted at several times with a 47-cc Ge(Li) detector. Concentration levels for the observed trace elements were determined by comparison to standards irradiated under the same conditions and dissolved in a few drops of appropriate solvent to give the same geometry as the samples. Three sets of crystals were analyzed and the results are shown in tables 43, 44, and 45. The results listed in table 45 were obtained on current production material. Detection limits for all the elements which could have been detected if present under these conditions were determined so that upper limits could be set for those elements not observed and these are given in table 46.

As can be seen from tables 44 and 45, some trace elements, such as $\mathrm{Ga}$, Ir, and the Cr dopant, appear to be fairly uniformly distributed within a given crystal. Others, especially $\mathrm{Ba}$, may show differences of more than two orders of 
Table 43. Results of activation analysis of ruby crystals, group I, ppm.

\section{Crystal Designation}

$\underline{\text { Element }} \quad \underline{050(4)} \quad \underline{050(9)} \quad \underline{075(4)} \quad \underline{075(9)} \quad \underline{100(4)} \quad \underline{100(9)}$

$\begin{array}{lllllll}\mathrm{Cr} & & & & \\ \left(\% \mathrm{Cr}_{2} \mathrm{O}_{3}\right) & 0.059 & 0.068 & 0.078 & 0.086 & 0.0104 & 0.098 \\ \mathrm{Ga} & 0.024 & 0.030 & 0.070 & 0.096 & 0.034 & 0.039 \\ \mathrm{Ir} & 0.040 & 0.037 & 0.033 & 0.038 & 0.023 & 0.033 \\ \mathrm{Ba} & 89 & 111 & 68 & 5.3 & 1.1 & 16 \\ \mathrm{Sr} & 2.5 & 3.0 & 1.9 & <0.1 & <0.1 & 0.5 \\ \mathrm{Ca} & 0.051 & 0.089 & 0.050 & 0.054 & 0.038 & 0.028 \\ \mathrm{Mn} & 0.0018 & 0.022 & 0.0023 & 0.0027 & <0.001 & <0.001 \\ \mathrm{~W} & 2.22 & 1.49 & 3.08 & 3.95 & 0.12 & 0.14 \\ \mathrm{Au} & 0.0028 & <0.0008 & 0.0014 & <0.001 & 0.0021 & <0.0003 \\ \mathrm{La} & 0.036 & 0.027 & 0.011 & <0.003 & <0.004 & <0.008\end{array}$

Table 44. Results of activation analysis of ruby crystals, group II, ppm.

$\begin{array}{lllll}\text { Element } & \frac{\text { Crystal "A" }}{\underline{1}} & \underline{2} & \frac{\text { Crystal "B" }}{1} & \underline{2}\end{array}$

$\begin{array}{cccccc}\left.\mathrm{Cr}_{\mathrm{Cr}} \mathrm{O}_{3}\right) & 0.0222 & 0.0215 & 0.0442 & 0.0463 & 0.0443 \\ \mathrm{Ga} & 3.05 & 2.98 & 0.014 & 0.016 & <0.004 \\ \mathrm{Ir} & 0.239 & 0.245 & 0.050 & 0.054 & 0.026 \\ \mathrm{Ba} & 42.6 & 0.24 & 15.2 & 1.26 & 9.4 \\ \mathrm{Sr} & 1.55 & <0.2 & 0.49 & 0.066 & 0.41 \\ \mathrm{Cu} & 0.075 & 0.062 & 0.026 & 0.060 & 0.070 \\ \mathrm{Mn} & 0.0013 & 0.002 & - & 0.0018 & 0.0012 \\ \mathrm{~W} & 0.032 & 0.052 & 0.015 & 0.0030 & 0.034\end{array}$


Table 45. Results of activation analysis of ruby crystals, group III, ppm.

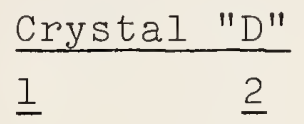

$\operatorname{Cr}\left(\% \mathrm{Cr}_{2} \mathrm{O}_{3}\right)$

$\mathrm{Ga}$

Ir

$\mathrm{Ba}$

$\mathrm{Sr}$

$\mathrm{Cu}$

$\mathrm{Mn}$
0.0373

0.008

0.045

0.28

$<0.03$

0.024

0.0008
0.0351

$<0.006$

0.041

1.74

0.085

0.016

0.0006

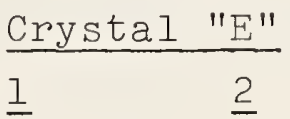

I

0.0389

0.0377

0.030

0.031

0.052

0.051

16.5

265

0.54

$8 \cdot 3$

0.027

0.137

0.0009

magnitude between adjacent pieces of the same crystal. To find out whether these extreme fluctuations could be caused by $\mathrm{Ba}$ segregation within the crystals, two of these adjacent pieces were analyzed for Ba by K. F. J. Heinrich and C. Fiori using electron probe microanalysis. In one of the pieces they found actual inclusions which contained $25-30 \% \mathrm{Ba}$.

A paper describing this work was presented at the 159 th National Meeting of the American Chemical Society, Houston, Texas, February 1970 and is now being prepared for publication. 
Table 46. Detection limits ${ }^{a}$ for trace elements in ruby, ppm.

\begin{tabular}{|c|c|c|c|c|c|c|}
\hline $\mathrm{Ag} \quad 0.05$ & $\mathrm{Eu}$ & 0.0005 & Mn & 0.0005 & $\mathrm{Sr}$ & 0.1 \\
\hline Ar 0.1 & $\mathrm{Fe}$ & 5 & Mo & 0.05 & $\mathrm{Ta}$ & 0.1 \\
\hline 0.002 & $\mathrm{Ga}$ & 0.003 & $\mathrm{Nd}$ & 1 & $* \mathrm{~Tb}$ & 0.2 \\
\hline 0.0001 & $\mathrm{Gd}$ & 0.02 & $\mathrm{Ni}$ & 1 & $\mathrm{Te}$ & 0.6 \\
\hline 0.1 & * Ge & 0.2 & *Os & 0.01 & *Th & 0.02 \\
\hline 0.01 & $\mathrm{Hf}$ & 0.001 & $\mathrm{Pd}$ & 10 & ${ }^{*} \mathrm{Tm}$ & 0.02 \\
\hline $\mathrm{Cd}$ & ${ }^{*} \mathrm{Hg}$ & 0.5 & $\mathrm{Pr}$ & 0.05 & ${ }^{*} U$ & 0.02 \\
\hline${ }^{*} \mathrm{Ce} \quad 0.1$ & Ho & 0.2 & $* P t$ & 0.03 & W & 0.01 \\
\hline $\mathrm{Cl} \quad 0.05$ & I & 0.01 & $\mathrm{Rb}$ & 1 & $\mathrm{Xe}$ & 0.01 \\
\hline 0.01 & In & 0.0001 & ${ }^{*} \operatorname{Re}$ & 0.001 & $\mathrm{Yb}$ & 0.01 \\
\hline $\mathrm{Cr} I$ & Ir & 0.005 & $\mathrm{Ru}$ & 1 & $\mathrm{Zn}$ & 0.5 \\
\hline Cs 0.5 & K & 1 & $\mathrm{Sb}$ & 0.02 & $\mathrm{Zr}$ & 100 \\
\hline 0.005 & $\mathrm{Kr}$ & 1 & $\mathrm{Sc}$ & 0.05 & & \\
\hline Dy $\quad 0.001$ & La & 0.003 & $\mathrm{Se}$ & 3 & & \\
\hline 0.005 & ${ }^{*} \mathrm{Lu}$ & 0.02 & $\mathrm{Sm}$ & 0.001 & & \\
\hline
\end{tabular}

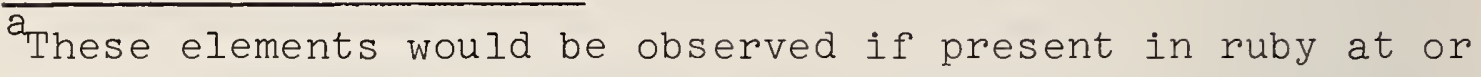
above the levels indicated, assuming the following conditions: $1 \mathrm{gm}$ sample, 1 hour irradiation at $10^{13} \mathrm{n} . \mathrm{cm}^{-2} \mathrm{sec}^{-1}$, 1 hour cooling time for decay of ${ }^{28} \mathrm{Al}, 10$ min counting time for short-lived activities ( $<2 \mathrm{hr}), 100 \mathrm{~min}$ counting time for long-lived activities ( $>2 \mathrm{hr}$ ), all counting done with 47-cc Ge(Li) detector. For elements marked * the limit will be about a factor of ten poorer in the presence of $\mathrm{Ir}$ and Cr at the levels normally observed to date. Elements not listed would not be detected under these conditions even if present.

(B. A. Thompson and E. C. Miller) 
4. Determination of Trace Elements in Synthetic Sapphire Crystals

At the request of the Inorganic Materials Division two pieces of a synthetic sapphire crystal were analyzed for trace element content by neutron activation analysis. The procedure followed was essentially the same as that previously described for the analysis of ruby crystals. The crystals were extremely pure and only a few elements could be observed. Their concentrations are given in table 47.

Table 47. Trace elements observed in sapphire crystals.

Element

$\mathrm{Cu}$

$\mathrm{Mn}$

Ir

$\mathrm{SC}$
Sample No. 1

$$
\begin{aligned}
& 0.027 \mathrm{ppm} \\
& \sim 0.0005 \mathrm{ppm} \\
& 0.002 \\
& \sim 0.015 \mathrm{ppm}
\end{aligned}
$$

Sample No. 2

$0.038 \mathrm{ppm}$

$\sim 0.0005 \mathrm{ppm}$

$0.0005 \mathrm{ppm}$

$\sim 0.015 \mathrm{ppm}$

For the elements not observed the upper limits listed in table 46 can be applied.

(B. A. Thompson)

5. Determination of Gold in Semiconductor Silicon by Neutron Activation Analysis

At the request of the Electronic Technology Division five silicon wafers were analyzed for gold by neutron activation. The most accurate possible values were required and therefore several special precautions were taken. The specimens as received were broken approximately in half to obtain samples which would fit horizontally in the irradiation container. Experiments by D. A. Becker (this report) have shown there is no significant flux variation ( $<1 \%)$ within this plane. Each sample (40-60 mg) was wrapped in polyethylene and a small piece $(\sim 0.5 \mathrm{mg})$ of $1 \mathrm{mil}$ gold foil was taped to 
Table 48. Results of analysis of silicon wafers.

Sample Designation

$1250^{\circ} \mathrm{C}$
$1150^{\circ} \mathrm{C}$
$1050^{\circ} \mathrm{C}$
$950^{\circ} \mathrm{C}$
$850^{\circ} \mathrm{C}$

Au Concentration, $\mathrm{gm} \mathrm{Au/gm} \mathrm{Si}$

$$
\begin{aligned}
& 1.10 \times 10^{-5} \\
& 3.35 \times 10^{-6} \\
& 6.37 \times 10^{-7} \\
& 7.41 \times 10^{-8} \\
& 2.89 \times 10^{-7}
\end{aligned}
$$

the outside of the polyethylene to serve as a standard. The samples were irradiated for 45 minutes in pneumatic tube RT4 of the NBSR which has the most highly thermalized flux of the available facilities (cadmium ratio( $\mathrm{Au}$ ) 100). The neutron flux was about $10^{13} \mathrm{n} \cdot \mathrm{cm}^{-2} \cdot \mathrm{sec}^{-1}$. After irradiation the samples were set aside for several days to allow any short-lived activities to decay away. Each sample was rinsed in $\mathrm{HNO}_{3}$ to remove surface contamination and then dissolved in a small volume of $\mathrm{HF}_{-\mathrm{HNO}_{3}}$. Each solution was made up to a standard volume and counted with a 47-cc Ge(Li) detector. The $0.412 \mathrm{MeV}$ peak of ${ }^{198} \mathrm{Au}$ was used for analysis and no $\gamma$-rays corresponding to any other radionuclides were observed. The gold foil standards were dissolved in aqua regia and a small aliquot of each was counted at the same geometry as the samples. The results are shown in Table 48. The precision of each value is estimated to be about $\pm 2 \%$ ( $95 \%$ C.L.) and the accuracy about $\pm 5 \%$ ( $95 \%$ C.L.).

(B. A. Thompson)

F. The Nuclear Track Technique

1. Introduction

The Nuclear Track Technique (NTT) is a method of analysis in which tracks produced by charged particles in plastic detectors are used for the determination of a few very specific elements. Usually the tracks are produced by either alpha particles (e.g. for the analysis of boron and lithium) 88 
or by fission fragments (e.g. uranium, and thorium). In most cases, the element to be determined is placed in intimate contact with a suitable plastic detector, the sandwich irradiated with either neutrons or photons, the plastic detector etched in a suitable etchant, and the track density compared to a standard [30] or the weight fraction of the element of interest calculated from known constants [31] using the following equation.

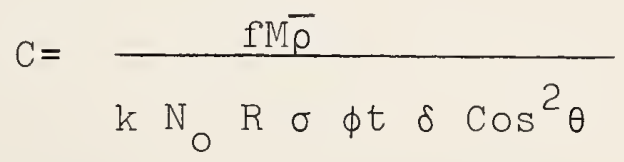

$f$ = multiplication factor ( 4 for boron, 1 for uranium)

$\mathrm{M}=$ Molecular weight of element determined

$\bar{\rho}=$ Average track density (tracks $\mathrm{cm}^{-2}$ )

$\mathrm{N}_{\mathrm{O}}=$ Avogradro's number

$\phi t=$ Thermal neutron fluence (neutrons. $\mathrm{cm}^{-2}$ )

$\mathrm{R}$ = Range of fission or alpha particle $(\mathrm{cm})$ in detector

= Thermal neutron cross section for ${ }^{235_{U}} \mathrm{n}(\mathrm{n}, \mathrm{f})$ or ${ }^{10} \mathrm{~B}(\mathrm{n}, \alpha)$ 7 Ii (cm)

$\delta=$ Density of material (grams. $\mathrm{cm}^{-3}$ )

$\cos ^{2} \theta=$ Etching efficiency factor; $\theta$ is the cone half angle at the tip of the etched track

$\mathrm{K}$ = Isotopic abundance of element determined

2. Analysis of Uranium and Boron in Trace Elements in

Glass

a. Experimental - Uranium Analysis

The trace elements in glasses to be analyzed were received in wafer form. They were selected cuts from 600 feet of glass canes. The glasses were doped with trace elements at three levels; $50 \mathrm{ppm}, I \mathrm{ppm}$, and $0.02 \mathrm{ppm}$. Prior to analysis, the glass wafers were cleaned by wiping the surfaces with alcohol and then placing the wafers in a cold dilute nitric acid solution ( 1 part $\mathrm{HNO}_{3}$ to 10 parts $\mathrm{H}_{2} \mathrm{O}$ ) for 5 minutes. After 
drying on a laminar flow clean bench, the wafers were weighed and measured. The $\sim 1 \mathrm{~mm}$ thick by $\sim 13 \mathrm{~mm}$ diameter wafers were placed on Lexan* polycarbonate slides, $20 \mathrm{~mm}$ wide $\times 40 \mathrm{~mm}$ long $\mathrm{x} 0.25 \mathrm{~mm}$ thick. The sample and detector were then bound together using paraffin wax. The resulting combination was enclosed in polyethylene bags to prevent external contamination, and irradiated in the NBS reactor at a thermal neutron flux of $1.1 \times 10^{13} \mathrm{n} \cdot \mathrm{cm}^{-2} \cdot \mathrm{sec}^{-1}$. The irradiation times were dictated by expected concentration of uranium in the wafers. After irradiation, the activity in the samples was allowed to decay for two half lives of ${ }^{24} \mathrm{Na}$, since the majority of the activity produced was from the $14 \% \mathrm{Na}_{2} \mathrm{O}$ content of the glass matrix.

After cooling, the plastic detectors were separated from the wafers, and were etched for 45 minutes at $50^{\circ} \mathrm{C}$ in

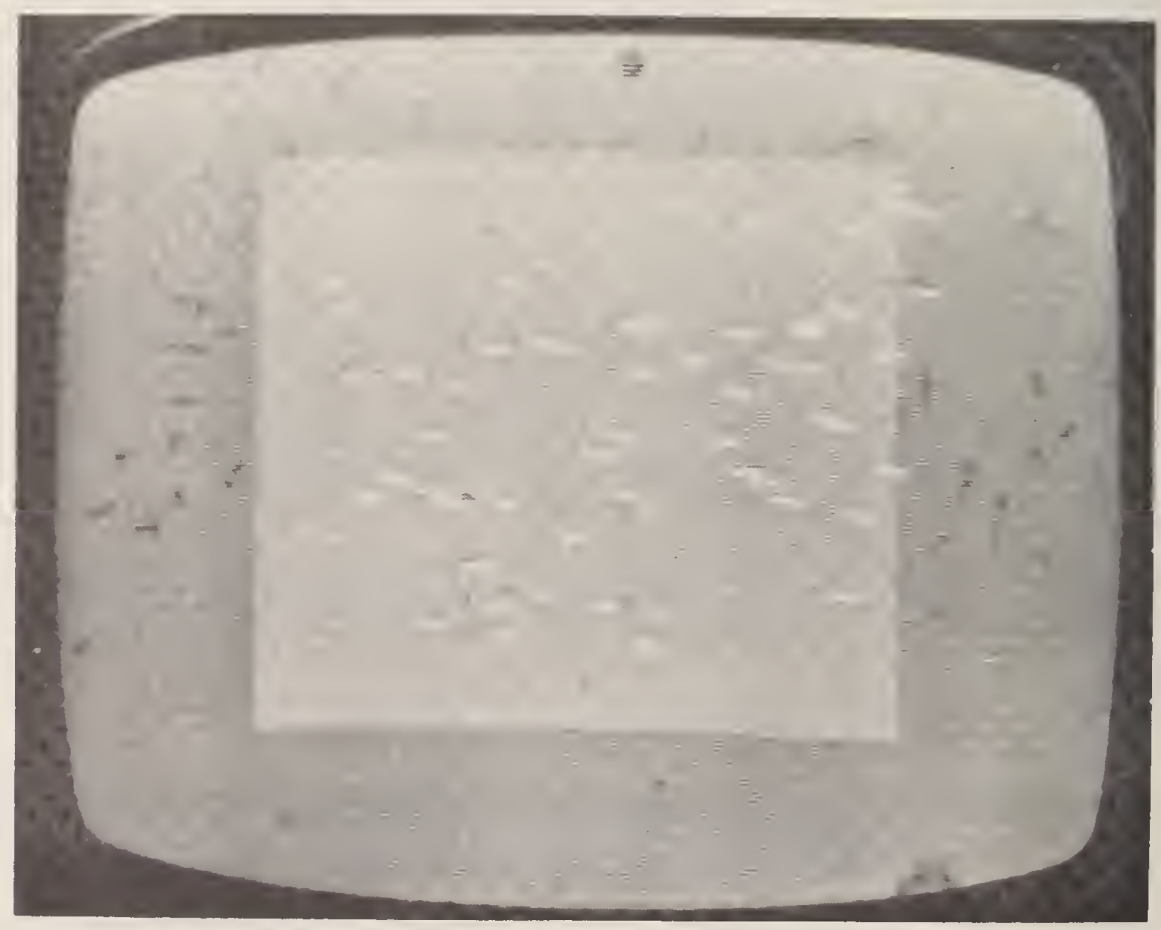

Figure 23. Microscope display for fission track counting. 
$6.5 \mathrm{~N} \mathrm{NaOH}$, rinsed in distilled water, and air dried. The etched detectors were mounted on microscope slides with tape. The tracks were counted with the use of a QTM quantimet* microscope. Using a magnification of $4800 \mathrm{x}$ on the monitor, fission track counts within a prescribed area were made in random fields of view (fig. 23).

During the course of analysis, however, it was discovered that the ${ }^{235} \mathrm{U}$ isotopic abundance in the glasses was different from natural uranium, i.e. the isotopic abundance was $0.238 \%$ for the $50 \mathrm{ppm}$ glass, $0.280 \%$ for the $1 \mathrm{ppm}$ glass, and $0.616 \%$ for the $0.02 \mathrm{ppm}$ glass. The analysis also indicated that the uranium was homogeneously distributed throughout the glasses. Typical results of the microscope fields observed are given in tables 49, 50, and 51.

Table 49. Boron and uranium content in the trace elements in glass (50 ppm).

Sample

$2 \mathrm{~A}$

$37 \mathrm{~A}$

$44 \mathrm{~A}$

$68 \mathrm{~A}$

$114 \mathrm{~A}$

Average
Boron Conc. (ppm)

$31.22 \pm 1.49^{a}$

$32.43 \pm 1.57$

$32.51 \pm 2.95$

$34.01 \pm 2.44$

$31.78 \pm 1.47$

$32.39 \pm 1.04$
Uranium Conc. (ppm)

$36.90 \pm 0.78$

$38.22 \pm 0.79$

$36.45 \pm 0.78$

$37.09 \pm 0.72$

$36.02 \pm 0.87$

$36.94 \pm 0.83$

$\overline{a_{ \pm}}$values are the standard deviation of the mean $(n=2)$.

Metals Research trade name for an image analyzing computer microscope. See disclaimer in Preface. 
b. Experimental - Boron Analysis

The boron analysis was similar to the uranium except that cellulose acetate butyrate ( $C A B$ ) detectors were used. The $\mathrm{CAB}$ was used to record the alpha track produced from nuclear reaction ${ }^{10} \mathrm{~B}(\mathrm{n}, \alpha)^{7} \mathrm{Li}$. These detectors were etched after irradiation for eight minutes in $6.5 \mathrm{~N} \mathrm{HaOH}$ at $70^{\circ} \mathrm{C}$. The boron distribution across the diameter of a wafer is shown graphically in figure 24. The average number of tracks across the diameter can be seen readily and is compared with the average value obtained from random fields of view taken across the entire wafer.

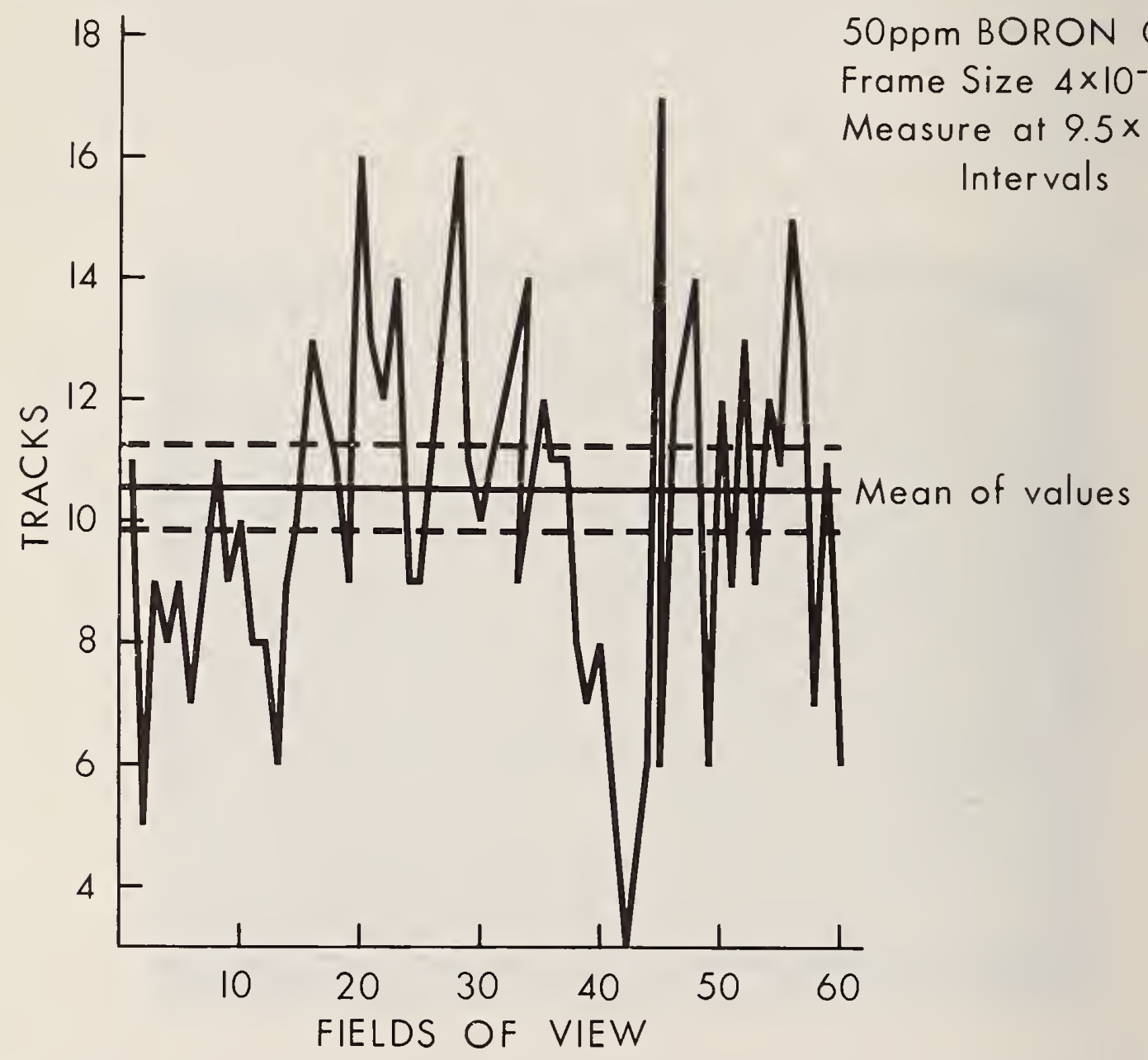

Figure 24. Boron distribution across the diameter of a glass wafer.

(B. S. Carpenter) 
Table 50. Boron and uranium content in the trace elements in glass ( $1 \mathrm{ppm}$ ).

Sample

$2 \mathrm{~A}$

$36 \mathrm{~A}$

$40 \mathrm{~A}$

$69 \mathrm{~A}$

$76 \mathrm{~A}$

$114 \mathrm{~A}$

Average
Boron Conc. (ppm)

$1.293 \pm 0.154^{\mathrm{a}}$

$1.251 \pm 0.201$

$1.379 \pm 0.135$

$1.287 \pm 0.086$

$1.296 \pm 0.135$

$1.227 \pm 0.160$

$1.295 \pm 0.052$
Uranium Conc. (ppm)

$0.839 \pm 0.021$

$0.835 \pm 0.019$

$0.853 \pm 0.031$

$0.801 \pm 0.029$

$0.818 \pm 0.025$

$0.824 \pm 0.021$

$0.828 \pm 0.018$

$a_{ \pm}$values are the standard deviation of the mean $(n=2)$.

Table 51. Boron and uranium content in the trace elements in glass $(0.02 \mathrm{ppm})$.

Sample

$2 \mathrm{~A}$

$33 \mathrm{~A}$

$43 \mathrm{~A}$

$51 \mathrm{~A}$

$61 \mathrm{~A}$

$76 \mathrm{~A}$

$130 \mathrm{~A}$

Average

$\overline{a_{ \pm}}$values are the standard deviation of the mean $(n=2)$.

\section{Boron Conc. (ppm) Uranium Conc. (ppb)}

$0.199 \pm 0.033^{a}$

$72.6 \pm 1.3$

$0.208 \pm 0.033$

$72.1 \pm 1.2$

$0.211 \pm 0.040$

$72.2 \pm 1.4$

$0.204 \pm 0.062$

$73 \cdot 5 \pm 2.2$

$0.206 \pm 0.058$

$72.0 \pm 1.1$

$0.195 \pm 0.037$

$0.198 \pm 0.019$

$0.203 \pm 0.057$

$72 \cdot 5 \pm 0.2$ 
3. Analysis of Orchard Leaves for Boron and Uranium

Previous work [30] had demonstrated that the analysis of biological material by the NTT was possible. In order to eliminate blank and standard problems present in the previously used methods, the direct determination method was used. In this method prior to analysis the orchard leaves were dried for 24 hours under vacuum at $90^{\circ} \mathrm{C}$. Then $250 \mathrm{mg}$ of the finely powdered leaves were pressed into $13 \mathrm{~mm}$ diameter pellets which were $1.5 \mathrm{~mm}$ thick, using 20,000 lbs of pressure from a hydraulic press.

The solid pellets were then bonded to plastic detectors with paraffin wax for irradiation. CAB was used for boron analysis and Lexan for uranium analysis. After irradiation the slides were etched using the conditions discussed above, and the average track density determined. The weight fractions are then calculated from the track densities. At present, the orchard leaves are still being analyzed.

(B. S. Carpenter)

4. Photo-fission Studies

Studies [32] have been continued on track production from high energy photon induced fission of $\mathrm{Bi}, \mathrm{Te}, \mathrm{Pb}, \mathrm{Th}$, and $U$. The first study entailed using different plastic detectors to determine if the detection sensitivity varied. Such detectors as Cronar* (a polyester), cellulose acetate, cellulose acetate butyrate, mylar, and Lexan polycarbonate were studied.

The second study was to determine the uranium weight fraction to total track response using bremsitrahlung produced by $35 \mathrm{MeV}$ electrons and a beam current of $20 \mu \mathrm{A}$. The response was linear over the interval taken as is indicated in figure 25.

* Dupont trade name for polyester. See disclaimer in Preface. 


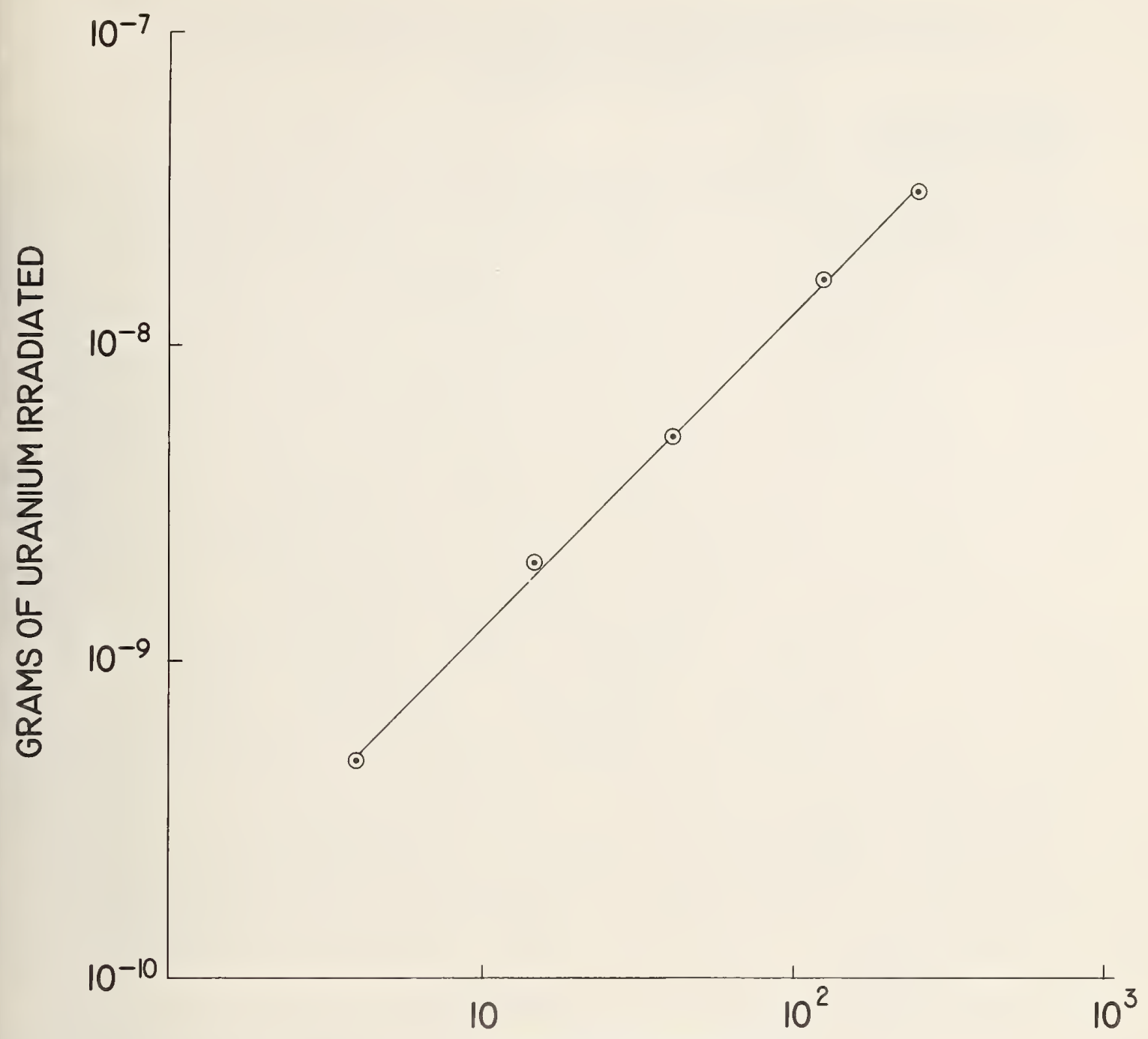

TRACKS OBSERVED

Figure 25. Photon activation study depicting uranium weight fraction to total track response.

Further work in this area will utilize both studies in attempts to determine trace quantities of various elements by photo-fission.

(B. S. Carpenter) 
4. ACTIVATION ANALYSIS WITH THE COCKCROFT-WALTON NEUTRON GENERATOR

\section{A. Introduction}

With a view to extending the usefulness of our neutron generator laboratory, several research studies initiated earlier [33] have been completed. These are; 1) Gamma-ray spectra and experimental sensitivities for 3-MeV neutron activation analysis; 2) 3-MeV neutron buildup interferences in 14-MeV activation analysis; 3) Thermalization characteristics of 3-MeV neutrons and 4) Pulse pile-up and nuclear interferences in $14-\mathrm{MeV}$ neutron activation analysis for trace oxygen. From an examination of the results reported herein it is now possible to obtain a broad perspective on the applicability of this type of neutron source for the solution of compositional problems in analytical chemistry. Typical examples illustrating the capability of the neutron generator as a versatile analytical tool are given. In this respect the generator has been applied for the certification of several organometallic standard Reference Materials in the analysis of which, thermalized neutrons, 3-MeV and $14-\mathrm{MeV}$ neutrons have been utilized.

In our continuing effort to establish a self-sufficient fast neutron activation facility, we have developed techniques and fabrication capability for reconditioning our own ion sources. In the past the maintenance of this critical unit involved a cost of several hundred dollars and a downtime of at least one month. Currently it is possible to recondition and replace key components of our Penning ion source and be functional within a few hours at a cost of a few tens of dollars. The ion source electrodes are fabricated in our shops from materials whose suitability was investigated in this laboratory.

As emphasized previously [33], olr awareness of the health physics problems associated with low-energy particle accelerators prompted a study of the possible neutron hazard 
resulting from the bombardment of blank targets with a hydrogen ion beam. Although hydrogen-on-blank experiments are routinely conducted during tune up operations, the possibility of a radiation hazard from neutron production by the interaction of the deuterium component of hydrogen gas with deuterons embedded in the target from previous irradiations, exists. Our results are indicative of the extent of this hazard and provide guide lines for experimenters in the safe operation of their individual facilities.

In conclusion, it is our opinion that the progress over the past four years has promoted this laboratory to the status of a self-sufficient and versatile fast neutron activation facility. The many investigations carried out and reported in our earlier progress reports [33,34,35] over this period of time have resulted in obtaining a sound analytical competence which has been successfully employed not only for the certification of standard materials, but to advance the state of the art significantly. In the latter instance our efforts in the achievement of high accuracy and precision have been successful such that it is possible to evaluate this technique in terms of other techniques of analysis.

(S. S. Nargolwalla)

B. Research Activities

1. Gamma-ray Spectra and Experimental Sensitivities for 3-MeV Neutron Activation Analysis

Past efforts in activation analysis with neutron generators have been primarily focused on the use of $14-\mathrm{MeV}$ neutrons because of the high yield from the D-T reaction. In certain applications, however, 3-MeV neutrons from the D-D reaction can be extremely useful. For example, interferences from nuclear reactions having thresholds above $3 \mathrm{MeV}$ are eliminated and the analysis fnr major components activated by 3-MeV neutrons can be easily performed. In addition, some elements have relatively high $3-\mathrm{MeV}$ neutron activation cross sections 
for $(n, \gamma)$ and $\left(n, n^{\prime} \gamma\right)$ reactions and are therefore about to be determined in the presence of matrix materials which would be highly activated by thermal or $14-\mathrm{MeV}$ neutrons. Perhaps one of the principal reasons for a comprehensive study on the 3-MeV neutron activation of the elements stems from the fact that 3-MeV neutron buildup with time during $14-\mathrm{MeV}$ neutron production could introduce significant systematic errors in $14-$ $\mathrm{MeV}$ neutron activation analysis. Therefore if the sensitivities for 3-MeV neutron activation were known in conjunction with the extent of $3-\mathrm{MeV}$ neutron buildup with time on the tritium target, it would be possible to estimate interferences from this mode of neutron production.

In this work, experimental sensitivities calculated from gamma-ray emission from 73 elements irradiated with $3-\mathrm{MeV}$ neutrons have been determined. Critical information, such

Table 52. Experimental sensitivity for 3-MeV neutron activation analysis.

Sensitivity Range

Counts/g $10^{6} \mathrm{n} \cdot \mathrm{cm}^{-2} \mathrm{sec}^{-1}$

Element

$$
\begin{aligned}
& \text { ca. } \quad 10^{8} \\
& \text { ca. } \quad 10^{6} \\
& >10^{5} \leq 10^{6} \\
& >10^{4} \leq 10^{5} \\
& >10 \leq 10^{4} \\
& <10^{3}
\end{aligned}
$$

$\mathrm{Au}, \mathrm{Hg}$

$\mathrm{Ag}, \mathrm{W}$

$\mathrm{Cd}$, In

$\mathrm{Sc}, \mathrm{V}, \mathrm{Mn}, \mathrm{Se}, \mathrm{Sr}, \mathrm{Y}, \mathrm{Te}, \mathrm{I}$,

$\mathrm{Cs}, \mathrm{Ba}, \mathrm{Sm}$, Dy, Er, Lu, Ta, Re, Ir, Pt.

$\mathrm{Al}, \mathrm{Cu}, \mathrm{Zn}, \mathrm{Ga}, \mathrm{Ge}, \mathrm{As}, \mathrm{Br}$, $\mathrm{Rb}, \mathrm{Mo}, \mathrm{Ru}, \mathrm{Rh}, \mathrm{Pd}, \mathrm{Sn}, \mathrm{Eu}$, $\mathrm{Gd}, \mathrm{Hf}, \mathrm{Tl}$.

$\mathrm{Be}, \mathrm{F}, \mathrm{Na}, \mathrm{Mg}$, Si, $\mathrm{P}, \mathrm{Cl}, \mathrm{K}$, $\mathrm{Ca}, \mathrm{Ti}, \mathrm{Cr}, \mathrm{Fe}, \mathrm{Co}, \mathrm{Ni}, \mathrm{Zr}$, $\mathrm{Nb}, \mathrm{Sb}, \mathrm{La}, \mathrm{Ce}, \mathrm{Pr}, \mathrm{Nd}, \mathrm{Tb}$, $\mathrm{Ho}, \mathrm{Tm}, \mathrm{Yb}, \mathrm{Pb}$.

Not Activated ${ }^{b}$ Li, C, N, O, S, Bi.

\footnotetext{
alo min. irradiation, 0.5 min. decay and a 10 min. count. Response less than twice background.
} 
as detection geometry, gamma-ray counting efficiencies and neutron source-sample configurations, has been provided to facilitate application in other neutron generator laboratories. A summary of the results has been reported [36]. A comprehensive paper describing the procedures in in press [37]. A general compilation of the gamma-ray spectra obtained in this study is available from the authors. In table 52 the experimental sensitivities for 3-MeV neutron activation analysis have been summarized.

From table 52, analytical applications for 3-MeV neutron activation can be predicted. Sensitivity ranges apply to gamma energy peaks of greatest abundance.

(S. S. Nargolwalla, J. Niewodniczanski, J. E. Suddueth)

2. D-D Neutron Activation Interferences in $14-\mathrm{MeV}$ Neutron Activation Analysis

A source of systematic error in $14-\mathrm{MeV}$ neutron activation stems from the build-up of $3-\mathrm{MeV}$ neutron flux. This buildup is due to neutron production from the interaction of the primary deuteron beam with deuterons embedded in the tritium target from previous bombardments. The extent of this buildup was studied using specially fabricated hydrogen-on-titanium targets of various hydrogen loadings. The effects of various parameters such as target loading and acceleration voltage were studied. The $3-\mathrm{MeV}$ neutron buildup as a function of time at a constant beam current was measured with a neutron monitor which had been previously calibrated as described earlier [33]. The results of the entire study are shown in figure 26 . It may be noted that after only $1-2 \mathrm{~mA}$ hour of integrated beam time the 3-MeV neutron flux extrapolated for a deuteron beam current of $2.5 \mathrm{~mA}$ is of the order of $10^{6} 3-\mathrm{MeV} \mathrm{n} \cdot \mathrm{cm}^{-2} \mathrm{sec}^{-1}$. In fact this flux is about the same as when the best deuteron targets were used in the sensitivity study described above.

From a knowledge of the build-up flux as a function of integrated beam time and $3-\mathrm{MeV}$ neutron activation sensitivities 


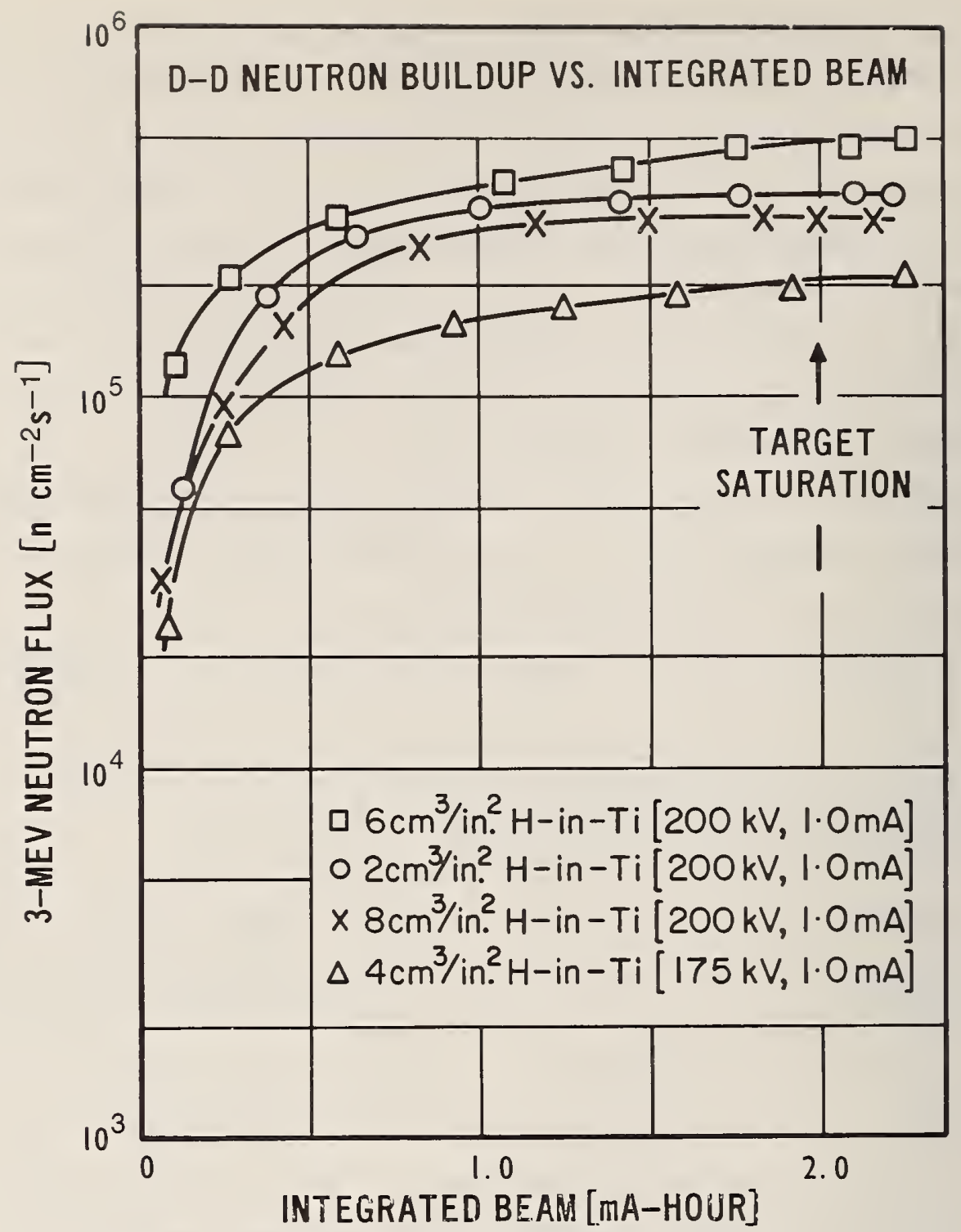

Figure 26. 3-MeV neutron buildup as a function of time at constant beam current.

(table 52), it is possible to estimate potential interferences in any procedure using $14-\mathrm{MeV}$ neutron activation. It is emphasized that the specific data apply only to the facility being used. However, the experimental concepts have been defined so that similar data can be obtained in a relatively short period of time for any other facility.

(S. S. Nargolwalla, J. Niewodniczanski and J. E. Suddueth)

3. Thermalization Characteristics of 3-MeV Neutrons

Activation analysis with neutron generators has been 100 
almost entirely limited to the use of $14-\mathrm{MeV}$ neutrons produced from the ${ }^{3} \mathrm{H}(\mathrm{d}, \mathrm{n})^{4} \mathrm{He}$ reaction, although some use of thermalized $14-\mathrm{MeV}$ neutrons has also been made. The fact that fluxes of $3-\mathrm{MeV}$ neutrons from the ${ }^{2} \mathrm{H}(\mathrm{d}, \mathrm{n})^{3} \mathrm{He}$ reaction are about two orders of magnitude lower than the $14-\mathrm{MeV}$ neutron flux has discouraged the use of the lower energy neutrons. Consequently, information in the literature regarding their use for activation analysis is very limited.

A recent study [38] relating to the analysis of mixed halogens in photographic emulsions suggested a detailed investigation into the applicability of $3-\mathrm{MeV}$ neutrons for the activation analysis of macroconstituents. In this report a summary of results obtained from a study of the thermalization characteristics of $3-\mathrm{MeV}$ neutrons using "limited" volume $\mathrm{H}_{2} \mathrm{O}$ and $\mathrm{D}_{2} \mathrm{O}$ moderator is given. The experimental arrangement was described previously [33].

Using bare and cadmium-covered indium foils, relative flux medsurements as a function of moderator thickness were made. The flux profiles obtained were used to define optimum moderator thickness for both moderators. Using this optimum thickness, measurements were made at the sample positions in our dual sample-biaxial rotating irradiation assembly [39] using gold and yttrium samples for the determination of slow $(<0.14 \mathrm{eV}$ ) and fast ( $>1 \mathrm{MeV}$ ) neutron fluxes respectively. Under these conditions estimates of sensitivities for thermal neutron irradiation can be computed.

Relative flux determinations as a function of moderator thickness are illustrated in figure 27. At thicknesses of approximately 6 and $8 \mathrm{~cm}$, the thermal fluxes reach a maximum for $\mathrm{H}_{2} \mathrm{O}$ and $\mathrm{D}_{2} \mathrm{O}$ respectively. Absolute neutron fluxes as measured at the sample position in our rotating irradiation device placed in contact with the moderator of optimum thickness were:

$$
\begin{array}{cc}
\mathrm{H}_{2} \mathrm{O}(6 \mathrm{~cm} .): \quad & 3.1 \times 10^{4} \mathrm{n} \cdot \mathrm{cm}^{-2} \mathrm{sec}^{-1}[<0.14 \mathrm{eV}] \\
& 3.6 \times 10^{4} \mathrm{n} \cdot \mathrm{cm}^{-2} \mathrm{sec}^{-1}[>1 \mathrm{MeV}] \\
& 101
\end{array}
$$




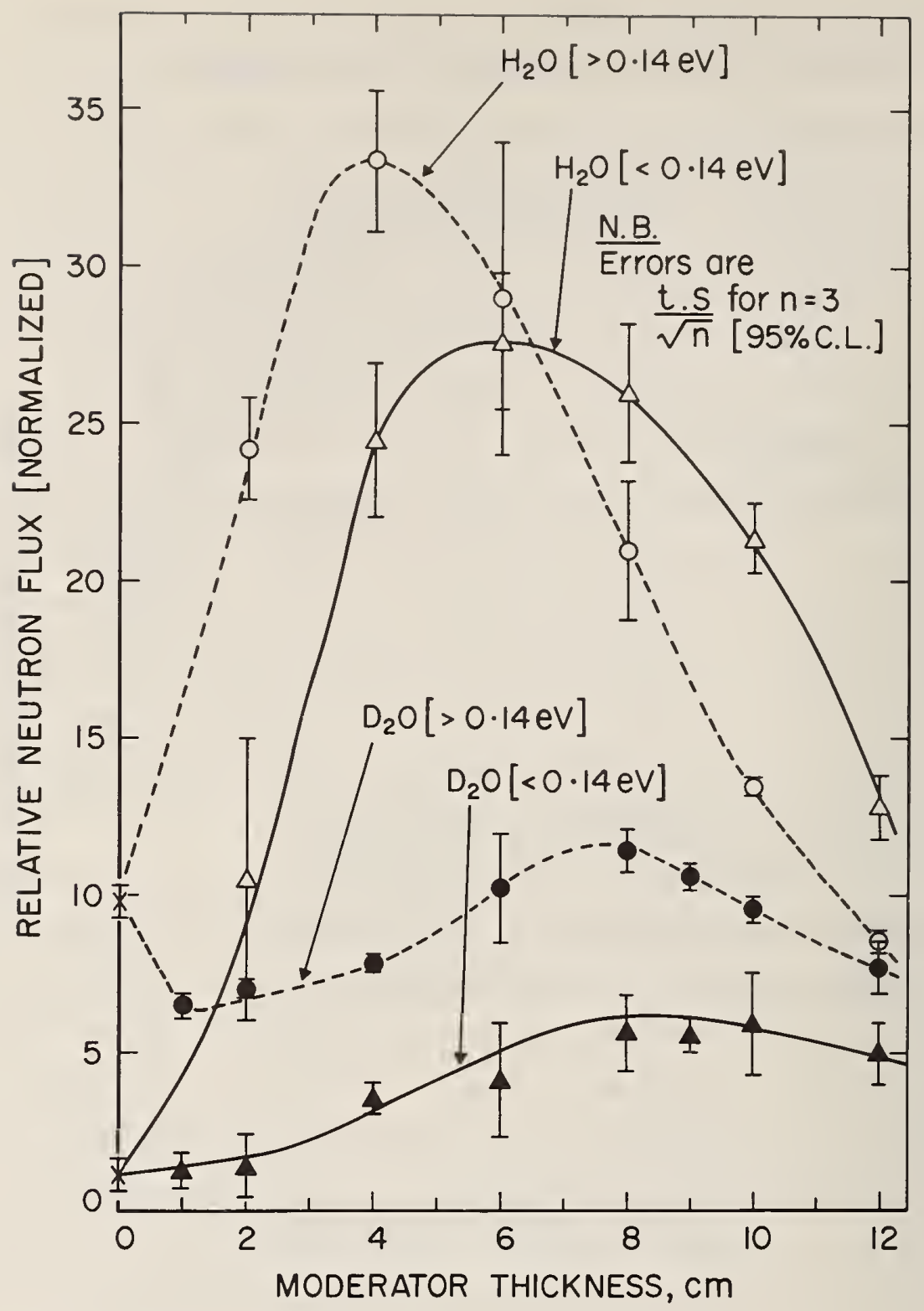

Figure 27. Relative flux determinations as a function of moderator thickness.

$$
\begin{aligned}
\mathrm{D}_{2} \mathrm{O}(8 \mathrm{~cm}): \quad & 6.2 \times 10^{3} \mathrm{n} \cdot \mathrm{cm}^{-2} \mathrm{sec}^{-1}[<0.14 \mathrm{eV}] \\
& 2.1 \times 10^{4} \mathrm{n} \cdot \mathrm{cm}^{-2} \mathrm{sec}^{-1}[>1 \mathrm{MeV}]
\end{aligned}
$$

Without moderator, 3-MeV neutron fluxes at the sample position of our rotating device located at 6 and $8 \mathrm{~cm}$ from the surface of the target cooling cap were $8.6 \times 10^{4}$ and $6.2 \times 10^{4}$ $\mathrm{n} . \mathrm{cm}^{-2} \mathrm{sec}^{-1}$ respectively.

(S. S. Nargolwalla, J. Niewodniczanski and J. E. Sudaueth) 102 
4. Pulse Pile-up and Nuclear Interferences in the Analysis for Trace Oxygen

In 14-MeV neutron activation analysis for trace oxygen, nuclear interferences from most matrix elements can be eliminated if counting of the high energy gamma emission from the ${ }^{16} \mathrm{O}(\mathrm{n}, \mathrm{p})^{16} \mathrm{~N}$ reaction is restricted to gamma energies greater than about $4.5 \mathrm{MeV}$. The well known $[40,41]$ exceptions are boron, fluorine and uranium. Oxygen determinations in these matrices does not follow the normal comparative analytical approach, and additional time-dependent matrix activation information must be obtained. Further, pulse pile-up contributions from highly activated matrix elements are caused by the inability of the normal scintillation detection system to register individual gamma photons emitted at a high rate. An earlier study [42] revealed pulse pile-up problems in the analysis of trace oxygen in aluminum and copper. In this report a comprehensive study of interferences for trace oxygen determination in 22 different elements is described. These elements serving as matrix materials were carefully selected on the basis of their importance to industry, their high 14-MeV neutron activation cross sections and unfavorable decay schemes in terms of short half-life, emission of high energy gamma photons and bremstrahlung production.

The general method for the evaluation of these interferences consisted of simultaneously irradiating the sample and standard in our rotating assembly, and sequential counting between two 4-in. x 3-in. NaI(TI) scintillation detectors. Benzoic acid served both as an oxygen standard and for flux normalization. Samples of the individual elements were prepared to conform closely to the geometrical configuration of the benzoic acid standard. The experimental sequence controlled by our sequence programmer included a 30 second irradiation, 4 second decay, 22 second count (sample), 4 second decay and a 22 second count (standard). This sequence was repeated three times with a time interval of 2 103 
minutes between sequences. The residual sample counts between the energy interval 4.8-8.0 MeV were accumulated in the multiscaler mode of the pulse-height analyzer and used to calculate the pulse pile-up contribution, and when possible, the half-life of the interfering nuclide.

A summary of results to be published [43] is shown in table 53. As expected the elements boron, fluorine and uranium present severe problems even when oxygen is present at levels of $1 \%$ or more. Interference from pulse pile-up is observed in the case of aluminum and copper. Therefore, at oxygen levels below 100 ppm in these elements, due consideration must be given to this pulse pile-up effect. Expected interferences from the 0.8 second isomers $90 \mathrm{~m} \mathrm{Zr}$ and $207 \mathrm{~m} \mathrm{~Pb}$ were not observed due to the 4 second decay prior to counting the sample. Finally, bremsstrahlung interferences resulting from the ${ }^{28} \mathrm{Si}(\mathrm{n}, \mathrm{p})^{28} \mathrm{Al}$ reaction was not observed because of the $2 \mathrm{~cm}$ thick lucite beta absorber employed in the design of our detector system.

Table 53. Results of trace oxygen interferences.

Matrix Interference ${ }^{a}$

Element

Boron

Fluorine

Aluminum

Copper

Uranium

$$
\begin{aligned}
& \frac{\text { Nuclear }}{1.3 \times 10^{4}} \\
& 4.9 \times 10^{5}
\end{aligned}
$$

20

\footnotetext{
For the elements $\mathrm{Be}, \mathrm{N}, \mathrm{Na}, \mathrm{Si}, \mathrm{P}, \mathrm{Ti}, \mathrm{V}, \mathrm{Cr}, \mathrm{Mn}, \mathrm{Fe}, \mathrm{Ni}$, $\mathrm{Zn}, \mathrm{Se}, \mathrm{Zr}, \mathrm{Mo}, \mathrm{Ba}$ and $\mathrm{Pb}$, interferences, if any, were not detectable
}

It is emphasized that the quantitative evaluations given in table 53 apply to the particular conditions used in our study. These conditions are normal in comparison to those 
prevailing during our routine oxygen determinations at the trace level. It is obvious that any significant alterations of the experimental set up will alter the data. However, the above investigation provides information which is useful for designing suitable experiments for the determination of trace oxygen in diverse matrices.

\section{(S. S. Nargolwalla)}

C. Analysis of Standard Reference Materials

In this area of analysis, the neutron generator was used to provide comparative data in the case of two organo-metallic SRMs. These were analyzed for their metallic component.

1. Cadmium Cyclohexane Butyrate SRM 1053a

Three samples drawn from the composite were analyzed. Each sample weighed approximately one gram. Individual samples were irradiated simultaneously with a standard of SRM 1053 (Certified $\mathrm{Cd}=24 \%$ ). Activation analysis of these samples was performed using $3-\mathrm{MeV}$ neutrons and counting the gamma

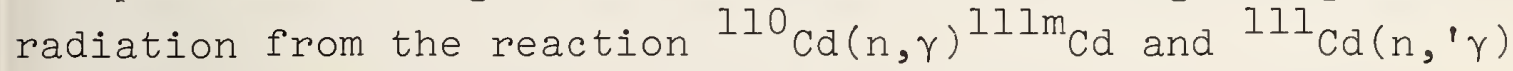
${ }^{1}{ }^{1} \mathrm{Cd}$. A result of $23.35 \pm 0.25 \%$ cd was obtained. The uncertainty is that of the mean of 3 samples at the $95 \%$ confidence level computed from $t s / \sqrt{3}$, where $s$ is the computed standard deviation of a single sample with (3-1) degrees of freedom.

\section{(S. S. Nargolwalla and J. E. Suddueth)}

2. Magnesium Cyclohexane Butyrate SRM 106lc

Six samples drawn from the bulk supply were analyzed. Sample weights were about one gram each. Each sample was irradiated with an equivalent weight standard (SRM 106lb; $\mathrm{Mg}=$ $6.53 \%)$. Activation of the samples were carried out with 14-MeV neutrons and counting the gamma radiation from the ${ }^{24} \mathrm{Mg}(\mathrm{n}, \mathrm{p}){ }^{24} \mathrm{Na}$ reaction. A value for the magnesium concentration of $6.73 \pm 0.05 \%$ was obtained. The uncertainty in this 
instance is of the mean of 6 samples at the $95 \%$ confidence level computed from $t s / \sqrt{6}$, where $s$ is the computed standard deviation of a single sample with (6-1) degrees of freedom.

(S. S. Nargolwalla and J. E. Suddueth)

D. Special Analyses

1. Oxygen in Metallic Barium

Two samples of metallic barium were received. Each sample as received was encapsulated in a sealed $8 \mathrm{~cm}^{3}$ polyethylene vial which was sealed inside a glass ampoule. The samples were analyzed inside the polyethylene vials using the standard 14-MeV neutron comparative technique with benzoic acid as the primary standard. Each sample was analyzed in duplicate and the results obtained are as follows:
Capsule \#l;
$0.356 \pm 0.007 \%$ Oxygen
Capsule \#2;
$0.358 \pm 0.007 \%$ Oxygen

The errors indicated are weighted mean standard errors of the weighted means based on Poisson counting statistics.

(S. S. Nargolwalla and J. E. Suddueth)

2. Oxygen in Niobium Metal and Niobium-Titanium Alloy

Small samples of $\mathrm{Nb}$ metal and $\mathrm{Nb}-\mathrm{Ti}$ alloy were analyzed for oxygen using the standard technique for oxygen in metals. Results for quadruplicate determinations obtained are given below.

Sample \#35 (Nb); $0.31 \pm 0.06 \%$ Oxygen

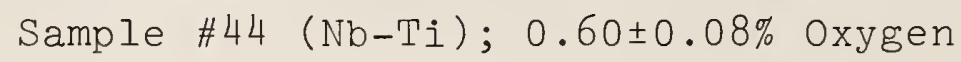

The errors quoted are the weighted mean standard errors of the weighted mean of quadruplicate determinations based on Poisson counting statistics. Corrections for all known sources of systematic errors have been accounted for in the final results.

(S. S. Nargolwalla and J. E. Suddueth) 
E. Status of the Neutron Generator Facility

1. Reconditioning the Penning Ion Source

In the past it has been our experience that reconditioning and/or repair of our Penning ion source was both an expensive and time consuming operation. We have therefore developed the capability for reconditioning our ion sources and can be operational within a few hours. In order to get satisiactory results from our ion sources, experiments were conducted to evaluate the life of several types of aluminum as suitable materials for the Penning source detectors. Our final conclusion was that both cathodes and anodes constructed from either 2024 or 6061 aluminum served adequately. Pure aluminum (type 1100) although possessing a high sputtering threshold burnt out within a few minutes at high beam current operation. It is expected that our reconditioned sources will operate satisfactorily in excess of 200 hours at a beam current of $2.5 \mathrm{~mA}$. A factor of ten reduction both in terms of cost and time has resulted from our own efforts to recondition our Penning ion source.

2. Health Physics

As mentioned in the introduction to this section, our concern in the practice of proper health physics precautions has always been paramount. It is well known that neutror generator operation manuals contain details for initial operation of the generator using a hydrogen beam on a blank plate of tantalum or copper. Our recent experiments concern the evaluation of a potential hazard from $3-M e V$ neutron emission. This hazard potentially exists since the small ( $0.015 \%$ deuterium) amount of deuterium contamination in hydrogen gas will generate 3-MeV neutrons by interacting with deuterium embedded in the target from earlier bombardments. Two types of experiments were conducted. In the first case hydrogen gas was allowed to bombard a deuterium loaded copper target, and in the second, hyarogen was allowed to bombard a new blank copper target. 
Initially a $2.5 \mathrm{~mA}$ deuterium beam was run on a clean copper target for approximately 3 hours. As expected the neutron flux built up steadily for the first 1 l/2 hours and then leveled off. The steady state neutron flux was measured to be approximately $1.4 \times 10^{5} \mathrm{n} . \mathrm{cm}^{-2} \mathrm{sec}^{-1}$ at the sample position in our rotating target assembly placed against the generator target. The deuterium bottle was now replaced by one containing hydrogen. The generator was then operated at a beam current of $2.0 \mathrm{~mA}$ for 2 hours. The measured neutron flux at saturation was about $1.8 \times 10^{3} \mathrm{n} \cdot \mathrm{cm}^{-2} \mathrm{sec}^{-1}$. The generator was shut down for about 20 minutes. The gas lines were evacuated and the vacuum pump allowed to pump down the entire system to $10^{-8} \mathrm{~mm} \mathrm{Hg}$.

The generator was then restarted and operated at $2.0 \mathrm{~mA}$ beam current. The measured neutron flux after an irradiation of 2 hours on a clean copper target approximately $100 \mathrm{n} . \mathrm{cm}^{-2}$ $\mathrm{sec}^{-1}$ at the sample position. Since the maximum permissable dose for 3-MeV neutron is of the order of $10 \mathrm{n} . \mathrm{cm}^{-2} \mathrm{sec}^{-1}$ the potential hazard at the target position is about 10 times greater. It is suggested as a general precaution that beam viewing operations during tune up be extremely limited at the target position.

(S. S. Nargolwalla and J. E. Suddueth) 
A. Facilities

During the year, a new irradiation facility for the NBS electron linear accelerator was designed and constructed. Irradiation facilities at the NBS LINAC are used for a variety of purposes other than photon activation analysis. These include the production of isotopes for Mössbauer and radiochemical separations studies, measurements of photonuclear yields from thick target bremsstrahlung for nuclear model interpretaiton, production of sources for decay scheme elucidation and EM isotope separator experiments, and measurement of high energy neutron and photofission yields of the heavier elements. Irradiations are conducted with electrons ranging in energy from 20 to $110 \mathrm{MeV}$. Planning of a new facility required the accommodation of all of these experiments, preferably permitting more than one irradiation to be performed at the same time.

An irradiation facility consists essentially of three components - a target made of high atomic number material in which electrons radiate to produce bremsstrahlung, a sample transfer system to send samples from the laboratory to the irradiation position and return, and a beam dump or backstop to absorb the intense photon beam. The irradiation assembly is shown in figure 28 .

1. Target System

A major consideration in the planning of an irradiation facility that is to accommodate a wide range of electron energies is the radiation target configuration. In practice one would desire a target that is just thick enough to absorb the electron beam. Since, however, the target will be irradiated by electrons of different energies, a compromise is necessary.

The bremsstrahlung target consists of six water-cooled l/16 in. tungsten foils. The foils are plated with a thin 


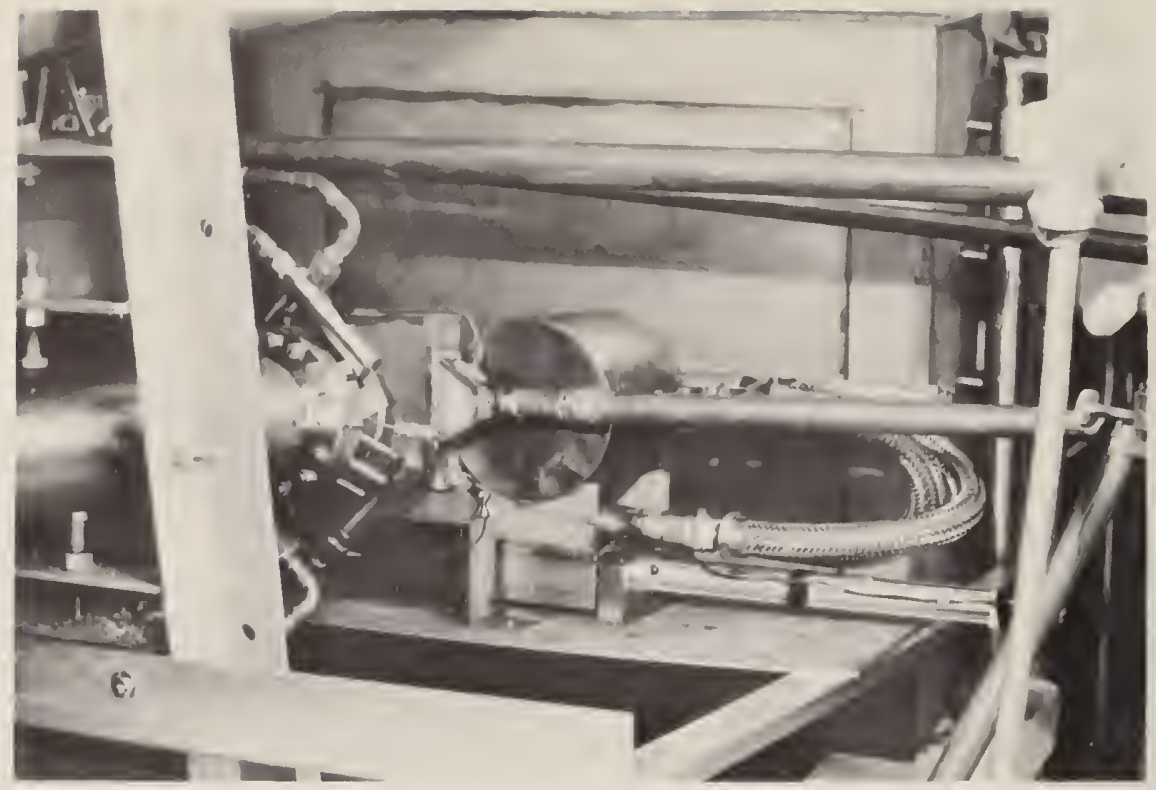

Figure 28. LINAC irradiation assembly.

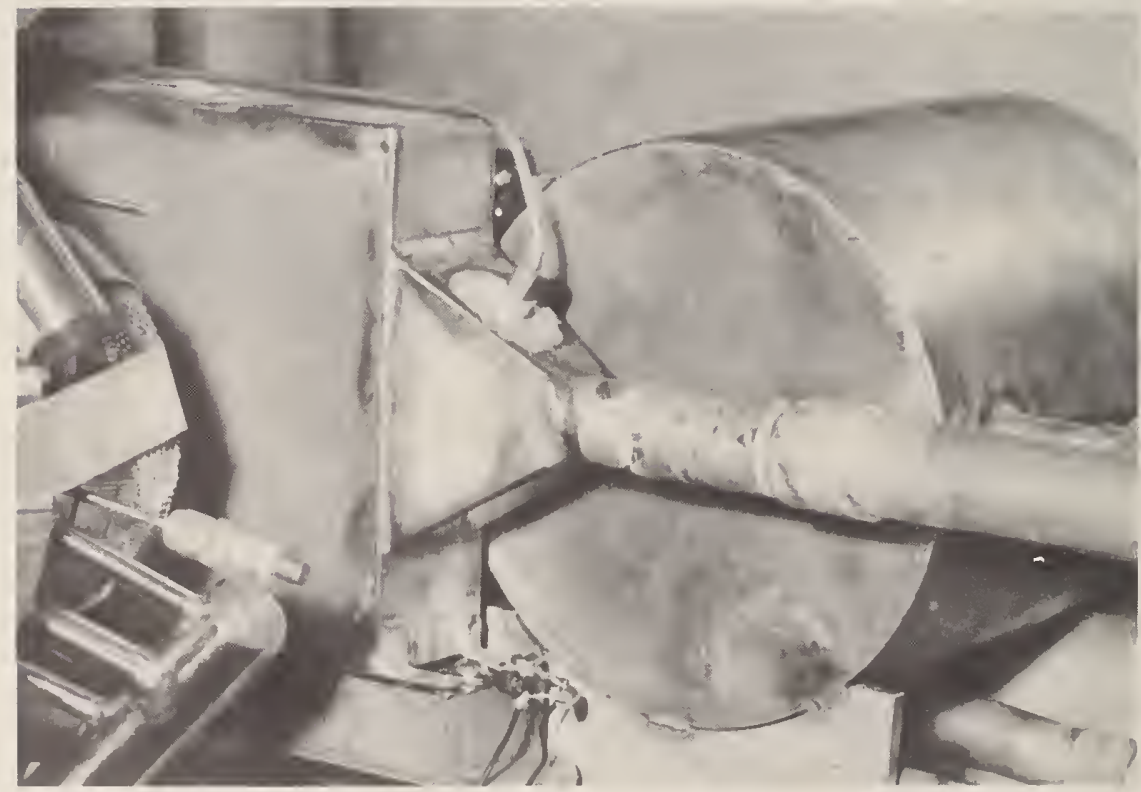

Figure 29. LINAC target assembly. 
coating of gold to minimize corrosion of the tungsten. The target is shown in figure 29.

Since it is difficult to weld tungsten to stainless steel, the plates were press fit into the grooves provided and the target holder welded shut. The one inch water inlet line minimizes direct channeling of the water through the center of the target. The front and back faces of the target holder are 0.15 inch thick stainless steel to reduce power deposition and subsequent heating by the electron beam.

This target will stop electrons of about $40 \mathrm{MeV}$ energies.

2. Sample Terminal

A dual irradiation terminal is located directly behind the target which is loaded pneumatically from the laboratory area in Service Area 2. One terminal is utilized for photon energies of up to about $40 \mathrm{MeV}$. For higher energies a watercooled aluminum block eight inches thick is moved directly behind the tungsten target to absorb unconverted electrons. The samples are loaded end on in the terminal. The terminal face is $15 \mathrm{mil}$ thick stainless steel to minimize absorption of photons.

\section{Beam Dump}

Behind the terminal assembly is a 34 in. $x 34$ in. x 8 in. stainless steel box which contains 52 two inch diameter lead filled copper tubes (fig. 30). This beam dump absorbs the intense photon beam and prevents it from striking structural material or equipment. A high volume of water is circulated around the tubes by a $11 / 2$ in. supply system.

4. Pneumatic Transfer and Shuttle Systems

In order to effectively irradiate samples in the target facility, a pneumatic transfer system was purchased from Reactor Experiments, Inc. of San Carlos, California* (fig. 31). The system consists of three separate air operated $7 / 8$ in. dia- 


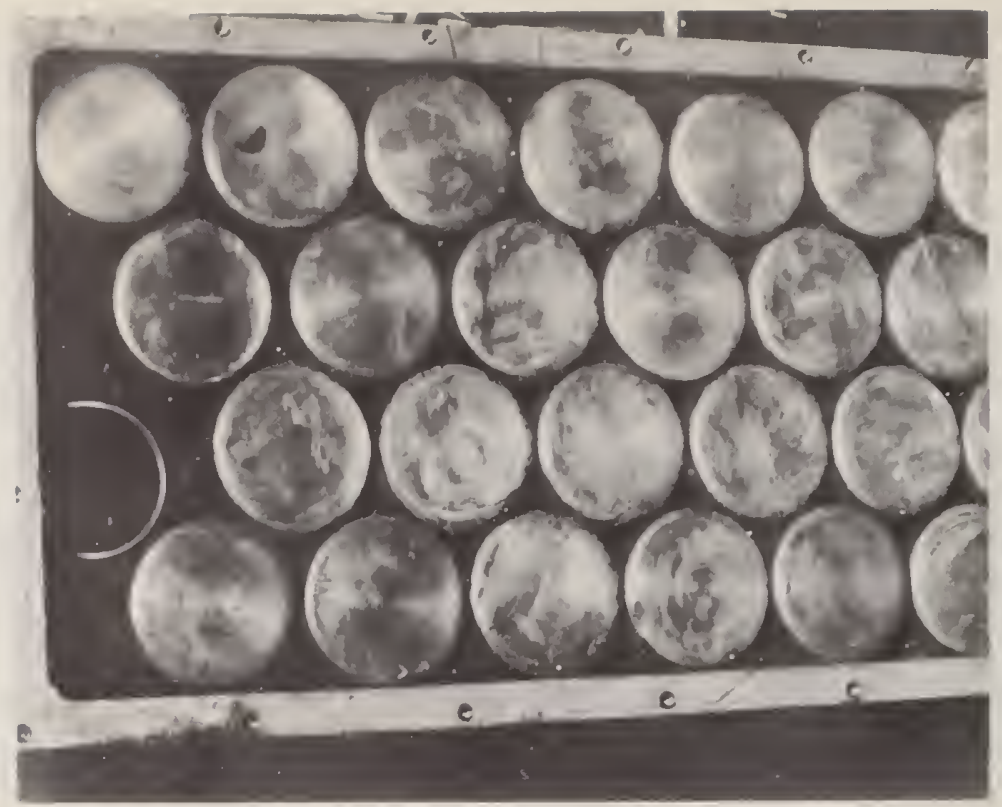

Figure 30. LINAC beam dump.

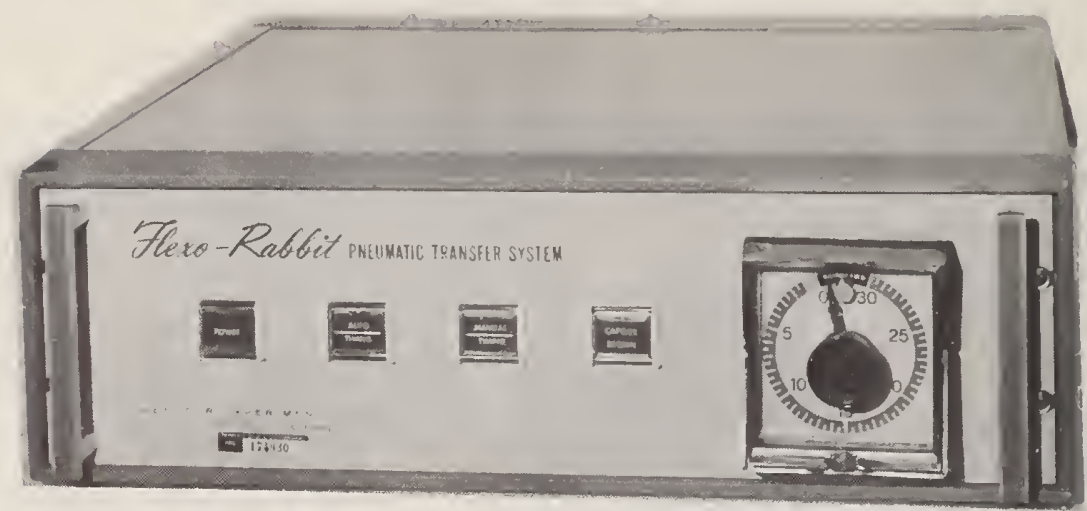

Figure 31. LINAC pneumatic transfer system control.

meter armoured polyethylene tubes. Two of these tubes are equipped to send a sample into the terminal, sense photoelectrically when it is near the tip, and then brake the samples' forward speed by the use of an air cushion arrangement. One of these two tubes will be connected to the low energy irradiation terminal while the other will be used in connection with a multi-position lazy suzan which will be designed and built at a later date. 
The third tube will be connected to the high energy irradiation terminal and will be loaded in much the same manner as the other two; however, at the discretion of the operator the sample may be sent to Isotope Separator Building instead of to the irradiation terminal, thereby providing a shuttle system from the Photon Activation Laboratory in Service Area \#2 of the LINAC Building to the Isotope Separator Building nearly $800 \mathrm{ft}$. away. Transit times are expected to be in the order of $10 \mathrm{sec}$. or less.

\section{LINAC Interface System}

In order that an electron beam can not be sent inadvertently to the photon activation target, a series of interlocks must be closed. These interlocks are to insure that there is cooling water flowing in all parts of the system and that no unusually high temperatures exist at the critical points. The various temperature and flowrate interlocks are tied into the accelerator by way of the LINAC Interface System (fig. 32). It is also possible with this unit to monitor the flow rate in G.P.M through the target and also the beam current through the target.

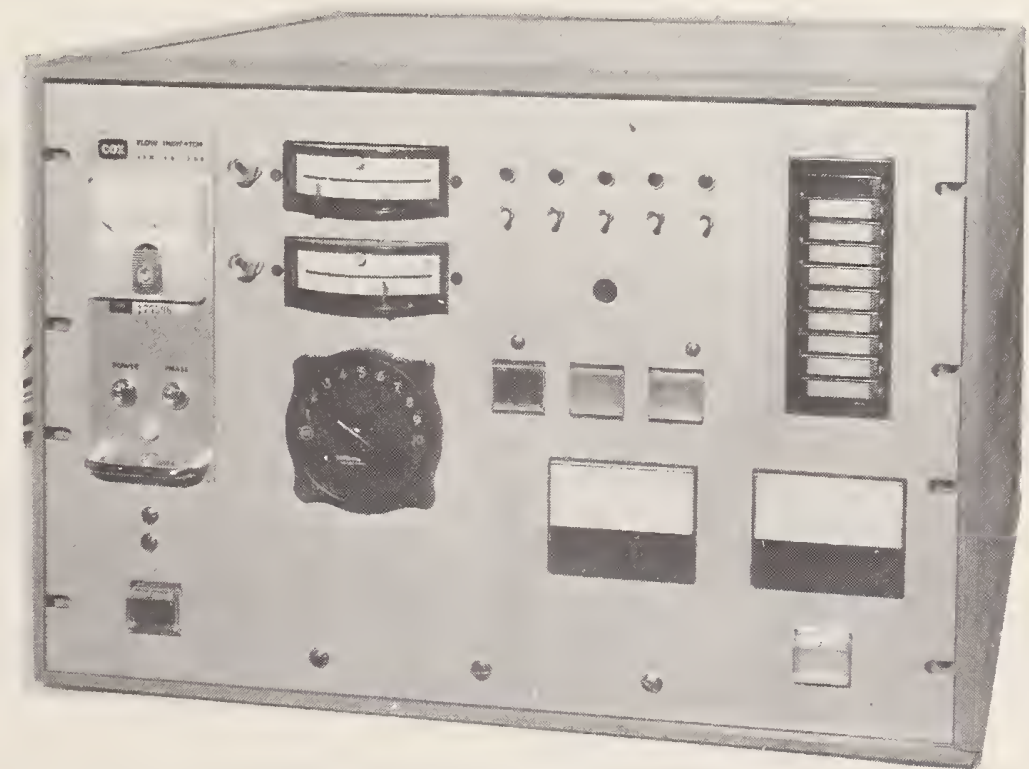

Figure 32. LINAC interface system control. 


\section{Pneumatic Tube Coupled Lazy Susan}

Plans have been made for a multi-position (lo or more) lazy susan to be located in close proximity to the target. This unit will have pneumatic tube capabilities and will be so constructed that samples can be inserted or removed at random. (G. J. Lutz, F. A. Lundgren and J. E. Suddueth)

B. Analyses

1. Determination of Thallium in Glass and Zinc Using Photon Activation

a. Introduction

Neutron activation analysis has been used for the determination of thallium in various substances such as zinc and zinc salts [44], potassium iodide [45], stone meteorites [46] and rocks [47].

Thermal neutron activation leads to 3.75 year ${ }^{204} \mathrm{Tl}$ and $4.3 \mathrm{~min} .{ }^{206} \mathrm{Tl}$, neither of which is a gamma emitter. The low cross section ( 0.11 barn) for the reaction ${ }^{205} \mathrm{Tl}(\mathrm{n}, \mathrm{\gamma})^{206} \mathrm{TI}$ and the short half-life of the product make this reaction less attractive for thallium determinations than the reaction $203_{\mathrm{Tl}}(\mathrm{n}, \mathrm{\gamma})^{204} \mathrm{Tl}$. The thermal neutron cross section for the latter reaction is about 11 barns.

The procedure described by Delberg et al. [45] utilizes the reaction ${ }^{203} \mathrm{TI}(\mathrm{n}, \gamma)^{204} \mathrm{Tl}$ with a sensitivity of approximately $0.1 \mu \mathrm{gm}$ thallium. The determination of pure beta emitters, however, requires rigorous chemical purifications and the long half-life of ${ }^{204} \mathrm{Tl}$ does not permit the observation of sample decay.

Fast neutron activation has also been applied to the determination of thallium [48], utilizing the reaction ${ }^{203} \mathrm{Tl}(\mathrm{n}, 2 \mathrm{n})^{202} \mathrm{Tl}$. Thallium 202 has a convenient half-life of 12.4 days and has a $0.44 \mathrm{MeV}$ gamma ray. The threshold for this reaction is in the order of 8-9 MeV and a relatively poor sensitivity of about $20 \mathrm{\mu gm}$ is obtained for an irradiation of 30 hours at a total flux of $10^{13} \mathrm{n} / \mathrm{cm}^{2} / \mathrm{sec}$. 
Thus the fact that thermal neutron activation produces pure beta emitters of thallium and fast neutron activation yields poor sensitivities make the determination of thallium by photonuclear activation most attractive. The work described here was undertaken to evaluate the potential for determining trace amounts of thallium in various matrices by the induction of the reaction ${ }^{203} \mathrm{Tl}(\gamma, n)^{202} \mathrm{Tl}$ with bremsstrahlung from an electron linear accelerator.

b. Chemistry of Thallium

Thallium has two stable oxidation states, univalent and trivalent, whose chemical properties differ markedly. Thallium (I) ions resemble the heavy alkali ions and lead and silver ions while thallium (III) ions are similar to indium and gallium ions. Thallium (III) is a strong oxidizing agent and forms complexes with various inorganic and organic liquids. The existance of two stable oxidation states constitutes the basis of a number of analytical methods. Various combinations of thallium (III) extraction with ethyl ether and isopropyl ether, TI (I) extractions with N-benzoyl-N-phenyl-hydroxylamine (BPHA) [49], and TI (I) precipitations of iodide and chromate have been used to obtain purified thallium in neutron activation analysis. A procedure has also been described for the separation of trallium (I) by amalgam exchange [50].

It was observed during the course of an investigation of a chelating agent that thallium (III) and gold (III) extracted quantitatively into benzyl alcohol and octyl alcohol from various concentrations of acid solution. Mercury (II) gave a partial extraction under these conditions (on the order of $35 \%$ extracted per extraction). Thallium (I) did not extract into either alcohol.

These extractions were applied to the chemical separation of thallium after irradiation by bremsstrahlung from the NBS electron linear accelerator in NBS standard glass samples and various zinc salts and zinc metal. 


\section{c. Irradiation Facility}

Most irradiations were performed in the $45^{\circ}$ facility of the NBS LINAC. The electron energy was about $35 \mathrm{MeV}$ with an average beam current of about $40 \mu \mathrm{A}$. Bremsstrahlung was produced by allowing the electron beam to radiate in a water cooled tungsten target. The converter, target and pneumatic transfer system have been described previously [51]. The samples were usually irradiated 1-2 hours.

An auxiliary irradiation facility has been placed in the magnet room of the NBS LINAC. The bremsstrahlung is obtained from extraneous scatter from the slit in another experimenters facility. The flux obtained in this facility is lower by a factor of about 10 than the facility previously described. The electron energy used in this facility is typically about $100 \mathrm{MeV}$. Although the higher electron energy makes the possibility of interfering reactions more probable, we feel much useful data has been obtained using this facility. Samples are transported from the laboratory to the irradiation facility and returned using a rabbit system which uses compressed air to facilitate transfer.

\section{d. Standards and Flux Monitors}

Samples of thallium sulfate were encapsulated in polyethylene snap cap vials of the same size as those used for the samples and were used as standards. Accurately weighed discs of copper were taped to each end of the vials as flux monitors and the vials were placed in screw cap aluminum rabbits. The $\mathrm{Tl}_{2} \mathrm{SO}_{4}$ standard was treated chemically in the same manner as the particular sample of interest.

\section{e. Experimental Procedure for the Determination of Thallium in Glass Samples}

Samples weighing approximately two grams were encapsulated in polyethylene snap cap vials and copper disc flux monitors were placed on each end of the vial. After encapsulation in a screw cap aluminum rabit the samples were irradi- 
ated in the $45^{\circ}$ facility of the NBS LINAC for 1-3 hours depending on the level of thallium in the sample.

The glass was dissolved in $10 \mathrm{ml}$ perchloric acid, with a few $\mathrm{ml}$ of $\mathrm{HF}$ and approximately $20 \mathrm{mg} \mathrm{Tl}_{2} \mathrm{SO}_{4}$ carrier added. Thallium (I) was oxidized to thallium (III) by the addition of a small amount of potassium chlorate solution. The mixture was diluted to $30 \mathrm{ml}$ with distilled $\mathrm{H}_{2} \mathrm{O}$, cooled to room temperature, and extracted twice with $15 \mathrm{ml}$ portions of octyl alcohol. The combined organic phases were washed three times with $6 \mathrm{~N} \mathrm{HCl}$ and the separator funnels centrifuged each time to facilitate the separation of layers. The organic phase was collected in a $2 \mathrm{oz}$. polyethylene bottle and counted using a $47 \mathrm{cc} \mathrm{Ge}(\mathrm{Li})$ detector. The $\mathrm{Tl}_{2} \mathrm{SO}_{4}$ standard was treated and extracted under similar conditions. The results obtained are reported in table 54.

Table 54. Results of analyses for thallium in NBS glass.

Sample Number

95GCG SRM 610

95GCE SRM 612

$95 \mathrm{G}$

SRM 614

$$
\begin{aligned}
& \frac{\text { Thallium (ppm) }}{\text { 66., 66., 62., 61., 61. }} \\
& \text { 15., 15., 12. } \\
& 0.20,0.24
\end{aligned}
$$

The use of a Ge(Li) detector permits the determination of thallium without a separation of gold. However, if desired, the gold can be readily separated before the extraction step by adding $\mathrm{Au}$ (III) carrier and passing $\mathrm{SO}_{2}$ through the solution to reduce gold to the metal and filtering. Thallium is reduced to thallium (I) during this step but may readily be oxidized again to thallium (III) and then extracted.

f. Experimental Procedure for the Determination of Thallium in Zinc and Zinc Salts

Samples weighing 1-3 grams were encapsulated in snap cap polyethylene vials and copper discs taped to each end to serve as flux monitors. The activation was performed in the 
auxiliary facility using another experimenters spray at a maximum electron energy of approximately $100 \mathrm{MeV}$. Since the flux in this facility was much lower than that obtained in the $45^{\circ}$ facility irradiation times of up to 8 hours were used.

The activated sample was dissolved in HCl and approxmately $20 \mathrm{mg}$ thallium carrier added. Thallium (I) was oxidized to thallium (III) by the addition of $\mathrm{H}_{2} \mathrm{O}_{2}$ and the sample heated to expel excess peroxide. The solution was diluted with distilled water to give a final HCl concentration of about IM. The sample was cooled to room temperature and extracted twice with $15 \mathrm{ml}$ portions of benzyl alcohol that had been pre-equilibriated with $1 \mathrm{~N} \mathrm{HCl.} \mathrm{The} \mathrm{separatory}$ funnels were centrifuged at 21000 rpm for 5 minutes to facilitate separation of layers and the organic phase washed at least five times with IN HCl with added zinc and copper carrier Some zinc which extracts under these conditions, is sometimes not completely washed from the organic phase. In these cases it is necessary to allow decay of the ${ }^{69 \mathrm{~m}} \mathrm{Zn}$ which has a 440 $\mathrm{keV}$ gamma ray - the same as that of ${ }^{202} \mathrm{Tl}$. The half-life of $69 \mathrm{~m} \mathrm{zn}$ is 14 hours.

The organic phase was counted on a $47 \mathrm{cc}$ Ge(Li) detector to obtain the thallium activity. The results obtained are presented in table 55.

Table 55. Thallium content in zinc and zinc salts.

Sample

Zinc Metal

Zinc Chloride

Zinc Acetate ppm Thallium

$1.5,2.0$

$2.5,2.5,3.5$

$7.0,8.0,5.4,6.7$

Octyl alcohol can also be used to extract the thallium in this procedure but the $\mathrm{HCl}$ concentration must be approximately $6 \mathrm{~N}$. Using octyl alcohol the thallium is not quantitatively extracted until the $\mathrm{HCl}$ concentration is $\sim 6 \mathrm{~N}$. But at this concentration a large amount of zinc is also extracted 
and problems were encountered in removing all the zinc by washings .

The spread in the results in table 55 can be attributed mainly to poor counting statistics. A typical sample of $7 \mathrm{ppm}$ thallium if counted in the Ge(Li) detector for 1200 min produces only 621 counts in the peak channels. The background for the same number of channels and for the same time is 468 counts. Thus, the net counts attributable to the sample in this instance have a standard deviation due to counting of approximately $21 \%$.

The sensitivity of this method should be increased significantly with the installation of a new target assembly that is in progress at the present time.

g. Interfering Reaction

A possible interference in the photon activation procedure for thallium is the nuclear reaction $204 \mathrm{~Pb}(\gamma, 2 \mathrm{n})^{202} \mathrm{~Pb}$. The ${ }^{202} \mathrm{~Pb}$ then decays by electron capture to ${ }^{202} \mathrm{Tl}$.

A sample of high purity $\mathrm{Pb}$ metal was irradiated in the $45^{\circ}$ facility at $35 \mathrm{MeV}$ for two hours to evaluate the possible $\mathrm{Pb}$ interference. The lead was dissolved, thallium carrier added, and thallium oxidized to the thallium (III) state with chlorate. The solution was extracted twice with $15 \mathrm{ml}$ benzyl alcohol and the combined organic phases washed three times with $1 \mathrm{~N} \mathrm{HNO}_{3}$. The organic layer was counted in a 47cc Ge( $\mathrm{ci}$ ) detector and compared to a $\mathrm{Tl}_{2} \mathrm{SO}_{4}$ standard that had been extracted into benzyl alcohol and counted under similar conditions. The sum of the counts in the thallium channels was not significantly more than the background indicated that interference from this source is negligible at $35 \mathrm{MeV}$.

(I. Masters and G. J. Lutz) 
2. Determination of Gold, Tantalum, Manganese and Iron in Cadmium Metal by Photon Activation

a. Introduction

Samples of one-fourth inch cadmium rod were received in the Section from the Office of Standard Reference Materials for determination of gold, tantalum, manganese and iron. Because of the extreme difficulties that would be encountered doing an analysis of cadmium by thermal neutron activation analysis due to neutron self-shielding, it was decided to accomplish the determinations by photon activation analysis.

\section{b. Experimental Determination of $\mathrm{Au}$ and $\mathrm{Ta}$}

The cadmium metal rod weighing approximately $4-5$ gms was irradiated in the NBS LINAC. Tantalum foil and gold foil were used as standards and were positioned so they surrounded the cadmium metal. The tantalum foil standard was dissolved in $H F$ and the gold foil in aqua regia for comparison with the sample.

The cadmium metal was dissolved in nitric acid, diluted to about $6 \mathrm{~N} \mathrm{HNO}_{3}$, and approximately $10 \mathrm{mg}$ Au carrier was added. The solution was extracted twice with $15 \mathrm{ml}$ benzyl alcohol that was pre-equilibrated with $6 \mathrm{~N} \mathrm{HNO}_{3}$. The separatory funnels were centrifuged for a few minutes at $1000 \mathrm{rpm}$ to facilitate the separation of layers. The organic phases were combined and washed 4-5 times with $6 \mathrm{~N} \mathrm{HNO}_{3}$ containing approximately $10 \mathrm{mg} / \mathrm{ml}$ cadmium. The aqueous phase and the washings were kept for the Ta analysis.

The combined organic phases were drained into a 2 oz. polyethylene bottle and counted for ${ }^{196} \mathrm{Au}$ using a $47 \mathrm{cc}$ Ge( Li) detector. An aliquot of the gold standard was also extracted into benzyl alcohol and counted under the same conditions as the sample.

The combined aqueous phase and the washings were made to 2M HF and extracted twice with $15 \mathrm{ml}$ methyl isobutyl ketone (MIBK). The tantalum is quantitatively extracted into MIBK 
under these conditions. The combined organic phases were washed several times with a mixture of $\mathrm{HNO}_{3}-\mathrm{HF}$ of the same concentration as the aqueous phase.

The organic phase was drained into a 2 oz. polyethylene bottle and counted on a $47 \mathrm{cc}$ Ge(Li) detector for $180 \mathrm{~m}$ Ta. This tantalum isotope has a half-life of 8.1 hours so the tantalum portion was counted before the gold portion. The tantalum standard was extracted and counted under similar conditions.

c. Determination of $\mathrm{Nin}$

The cadmium metal rod weighing approximately $4-5$ gms was irradiated in the NBS LINAC. Manganese metal chips were placed around the sample and were used as the standard.

After irradiation the cadmium metal was dissolved in $6 \mathrm{~N} \mathrm{HNO}_{3}$ and the solution heated. Approximately $100 \mathrm{mg} \mathrm{Mn}$ carrier followed by sodium bromate were added. The manganese was precipitated under these conditions as $\mathrm{MnO}_{2}$. The precipitate was collected on filter paper using a Buchner apparatus and washed several times with $6 \mathrm{~N} \mathrm{HNO}_{3}$. The $\mathrm{MnO}_{2}$ was then dissolved in $3 \mathrm{~N} \mathrm{HCl}$ and silver nitrate added to precipitate AgCl. The mixture was filtered and the precipitate discarded. This precipitation was repeated several times to remove the various silver isotopes formed from stable cadmium isotopes during the irradiation. The solution and the washings were combined and evaporated to $30 \mathrm{ml}$ and this solution counted on the $47 \mathrm{cc}$ Ge(Li) detector. An HCI solution of the manganese standard was counted under conditions similar to those of the sample. The sum of the counts in the channels of the $0.84 \mathrm{MeV}{ }^{54} \mathrm{Mn}$ peak were used to quantitate the results.

b. Determination of Fe

The cadmium metal rod surrounded by chips of the Fe foil was irradiated and the reaction ${ }^{57} \mathrm{Fe}(\gamma, \mathrm{p}){ }^{56} \mathrm{Nin}$ was used for the determination of iron. 
The sample was dissolved in $6 \mathrm{~N} \mathrm{HNO}_{3}$ and the solution heated. Approximately $100 \mathrm{mg}$ Mn carrier followed by sodium bromate were added. The manganese was precipitated as $\mathrm{MnO}_{2}$ and the precipitate collected by filtration. The $\mathrm{MnO}_{2}$ was dissolved in $\mathrm{HCl}$ and $\mathrm{AgCl}$ precipitations as in the procedure for manganese.

The resulting solution was evaporated as rapidly as possible to $\sim 30 \mathrm{ml}$ and the $0.845 \mathrm{MeV}$ peak of $2.58 \mathrm{hr}{ }^{56} \mathrm{Mn}$ used to quantitate the manganese. The iron standard was dissolved in $\mathrm{HNO}_{3}$ and the manganese precipitated as MnO using $\mathrm{NaBrO}_{3}$. The precipitate was dissolved in $\mathrm{HCl}$ and the solution counted under the same conditions as the sample for ${ }^{56}$ Mn activity.

(G. J. Lutz and L. Masters)

3. Determination of Nitrogen in Metals by Photon Activation

Preliminary experiments are being conducted to develop reliable techniques for the determination of nitrogen in metals by photon activation analysis. The reaction induced is ${ }^{14} \mathrm{~N}(\gamma, n)^{13} \mathrm{~N}$. The product nucleus decays by positron emission and has a half-life of 10.0 minutes.

Generally, the most difficult problem in developing methods for determination of the light elements by photon activation is assuring a clean quantitative separation. It appears that for many metals, after irradiation and dissolution in an oxidizing medium, the nitrogen is present as ammonium ion. This can be liberated as ammonia by addition of concentrated sodium hydroxide. It is difficult to distill all of the radioammonia quantitatively in the relatively short period of time required so a known amount of ammonia was added before the sodium hydroxide. The sodium hydroxide is added to the solution in a closed glass system and the solution is heated and agitated for several minutes. Radioammonia is trapped in a known amount of sulfuric acid. After counting the annihilation radiation from the ${ }^{13} \mathrm{~N}$ by 
coincidence spectrometry with $2 \mathrm{NaI}$ detectors, the sulfuric acid is back-titrated to determine the yield. Yields of 60-80\% are typical.

The standards are ammonium sulfate. After irradiation, the standards are placed in a special aluminum counting container of sufficient thickness to annihilate all of the positrons in a small volume. They were counted with one detector only at a distance of about eighteen inches. The counting container was then filled with copper powder in which ${ }^{64} \mathrm{Cu}$, a positron emitter, had been induced. This was counted at exactly the same geometry as the nitrogen standards. The copper powder was then dissolved in nitric acid and an aliquot of the solution counted under the same conditions as the sample. The ratio of the counting rate of the ${ }^{64} \mathrm{Cu}$ allowed a computation of the counting correction factor between samples and nitrogen standards.

Fluxes are monitored with copper. The reaction ${ }^{63} \mathrm{Cu}$ $(\gamma, n){ }^{62} \mathrm{Cu}$ is induced. ${ }^{62} \mathrm{Cu}$ has the same half-life as ${ }^{13} \mathrm{~N}$ and is thus very satisfactory for this purpose.

A few runs have been made on some NBS Standard Reference Materials already certified for nitrogen. These were in the form of chips. The results for ferrous alloys were within $15 \%$ of the certified values, which is moderately acceptable at this preliminary stage of our investigations. Alloys of titanium and zirconium gave unreasonably high values. This is attributed to recoil nitrogen in the air. The chips are too small to etch. Inert atomsphere facilities for packaging will be required.

(G. J. Lutz and L. Masters) 
6. APPLICATION OF COMPUTERS TO ACTIVATION ANALYSIS

\section{A. Introduction}

Assistance from computers can be of great benefit in data handing and computation of results for activation analysis, thereby relieving the experimenter of much tedious work. The application of computer techniques to achieving this goal is described in this section of the progress report. Before proceeding with the description of what has been accomplished, it may be valuable to present an overview of some of the problems in data handing and result computation, and to indicate how these problems are partially or completely solved by computer techniques.

Computers are useful in two distinct, but not unrelated, areas of activation analysis data processing. These areas, alluded to above, may be labeled data acquisition and manipulation (DAM), and result computation (RC) or computation of results (COR). The first area (DAM) could also be called data handling, but this often implies result computation also. Because the two areas are actually quite distinct, they should have distinct labels. Therefore, what we shall call data acquisition and manipulation refers first of all to retrieval of data from experimental apparatus; for example, the recording on magnetic tape of a spectrum from a multichannel analyzer is one type of retrieval. This step in process is the acquisition of the data.

Once the data has been acquired, hopefully in a form compatible with the computer to be used, it may be stored for use at a later date, or it may be processed by the computer for result computation. It is likely that the data, as originally recorded, will need further manipulation before the result computation can begin. This manipulation involves translation of the data into appropriate form for use internally by the computer. Additional manipulation may be required since the initial data recording may contain errors, 
and these errors must be taken into account before processing the data for result computation. Manipulation may also be performed to create permanent records of certain data, such as catalog spectra. The term data acquisition and manipulation, therefore, includes readout of the data from the analytical instrument, storage of the data, correction of errors in the record, translation into internal computer format, and permanent file generation. After completion of the data acquisition and minipulation phase, the data has been stored in the core of the computer as real or integer numbers. Result computation then proceeds by the location of peaks, computation of peak areas, calculation of concentration (e.g. parts per million), performance of decay curve analysis, and so on. Some of the advantages of digital computers for data acquisition and manipulation will become apparent from the following example. Suppose an analyst takes a spectrum using a Ge(Li) detector and a 4096 channel analyzer. If the memory capacity is six decimal digits (i.e. 0-999999) then each experiment produces $6 \times 4096$ or 24576 digits of data. If lead zeros are suppressed, the number of digits to be considered is significantly reduced, but, even so, a teletype printout of 4096 channels of data occupies 512 printed lines and about 0.75 hours of readout time while a paper printer will give 4096 lines; clearly, this is a very cumbersome amount of data to record, store, or even scan visually. If the spectrum is a complicated one, it may have as many as 50 peaks, and it will be a tedious, time consuming chore to locate the peaks in which the analyst is interested. It is, therefore, very advantageous to let a computer help with the analysis. The computer can easily cope with the 24576 digits, assuming the analyzer has furnished the data in computer compatible form. Once the spectrum is in core, the computer can quickly search for peaks, calculate peak areas and print the peak locations and areas. 
Having briefly indicated some of the advantages of computer processing of activation analysis data, it is pertinent to discuss some of the problems.

The first problem is acquisition in computer format of the information accumulated by the radiation measuring device. If an appreciable volume of data is accumulated, i.e. by a multichannel analyzer, readout onto magnetic tape is often available. These readouts are not necessarily in a convenient format for utilization in the computer. Records written on magnetic tape by multichannel analyzers are usually too long to be handled by FORTRAN, since the inputoutput buffer is arranged to handle one line of print, 132 characters. Two of the analyzers used by the Activation Analysis Section write the data in blocks of 1542 characters (256 channels of data plus one tag word, 6 characters per word or channel). Fortunately, the UNIVAC 1108 software subroutine, NTRAN, makes it easy to read the longer tape records; it should be pointed out, however, that previous experience with an IBM-360 was very different, for it took several months, several hundred dollars, and several man weeks to read a similar tape with our own assembly language program. Reading a block of data with NTRAN gives an array of the UNIVAC equivalent of BCD characters. These characters must then be converted into integer or real numbers. Illegal characters on the tape can be a problem here.

Once the data are in core and ready for some sort of numeric processing, we have to contend with statistical scatter in the data and with the fact that no mathematical function exists which describes more than a small region of a Ge(Li) detector spectrum. The numerical analysis of gamma ray spectra has received considerable attention from many scientists, and fairly good progress has been made [52]. Considerable progress is needed in estimation of minor components 
in Ge(Li) detector spectra and in decay curve analysis, however.

In particular, the computation of net full energy peak areas is a very important one for activation analysis, since many activation analysis determinations utilize peak areas obtained from Ge(Li) or NaI(TI) spectra. The problem lies in the calculation of base area, which is subtracted from the gross area, to obtain the desired net peak area. If the net area is large, relative to the base area, there is no problem because a significant error in the base area will not result in a significant relative error in the net area. Very often the opposite situation occurs, however, and we need to base quantitative results on peaks which have small net areas compared to base areas. In this case, shape and height of the baseline are crucial. Some methods of studying this problem have been published [53, 54], but further study is still needed.

Other topics which need study are complex peak resolution, decay curve analysis, and component identification. An example of interfering peaks which would benefit from numerical resolution is the interference between the $559 \mathrm{keV}$ 76 As peak and the $564 \mathrm{keV}{ }^{122} \mathrm{Sb}$ peak. These isotopes are frequently found together, especially in the analysis of steels.

Our group sometimes analyzes samples about which little is known concerning chemical composition. It would be verty useful if we had a computer routine to examine the Ge(Li) detector spectra and identify the spectral components. Particular attention must be given, in this type of analysis, to those components which are obscured by other, more intense components, and to those components which are present at amounts just above their detectable limits.

In the foregoing paragraphs a brief summary of areas of application of computer techniques to activation analysis has been given. Some of the problem cases have been outlined 
roughly. In the remainder of this section the progress that has been made since this work was begun last October (1969) is discussed.

(H. P. Yule)

B. ALSPIS - Activation Analysis Peak Area Program

ALSPIS is a Fortran V computer program which locates peaks in Ge(Li) detector spectra, and computes their areas, widths, and energies. Data are read from cards or from magnetic tape, and checked for errors. Various options allow the analyst considerable control over the course of the subsequent calculations. Details of the program are indicated below. The program is by no means finished, for it will have to be changed to meet the changing requirements of the activation analysis group, and the basic algorithm can be modified, improved, extended, or expanded with new ideas; or a new alogrithm can be written.

ALSPIS (pronounced: all-spice) may be considered a two part program with the addition of a third part to control program flow. Data are read into the computer and manipulated by part one, and result computation is performed in part two. The three parts will be described below in order.

The data manipulation may be best described in terms of its development. At the outset, our group had a TMC-4096 channel analyzer coupled to a DATAMEC* magnetic tape unit. All that was known about the form of the data on the tape other than the density, 200 BPI, was that a four digit tag word was written on the tape. Operation of the units had been limited to using the tape for storing spectra which could later be read back into the analyzer.

Inspection of the tape unit indicated that it probably wrote $B C D$ characters on the tape, and using the utility routine, TPRINT, available at the NBS UNIVAC-1108, it was found that each channel of the data (data word) is represented by six BCD characters and that each record on the

*See disclaimer in Preface. 
tape consists of an unbroken string of 257 words. The first two characters of the first word in a record are the address of the 256 channel memory segment in the analyzer, and the next four are the tag word which the analyst set at the time of recording. The remaining 256 words are data. If more than 256 channels of data are written on the tape, the analyzer records them in groups (records) of 257 words as described above - a 2048 channel spectrum contains eight such groups, for example. The parity of the recording is even.

As indicated in the introduction, it is not possible to read the 1542-character records with a FORTRAN READ statement since it is limited to about 132 characters. However, the utility subroutine NTRAN* may be used for this purpose, and the 1542-character record is stored in an array of 257 words of 6 characters each. The next step is to convert them from a string of characters to real numbers.

An easy way to accomplish this is to write them onto a magnetic drum scratch area, using a FORTRAN write statement with an alphanumeric format, rewind the drum, and read the data back into core using a FORTRAN statement with a numeric format. The program simply repeats the process as many times as necessary to read a complete spectrum.

Data manipulation in this way is sufficient to provide numbers for result computations unless errors occurred when the tape was written. These errors are of two possible types. The first type is the transcription of a non-numeric character by improperly functioning electronics, and the second is transcription of records which are not the prescribed length of 1542 characters. The second type of error is easily overcome by programming to ignore records not having 1542 characters. Most of the records so ignored are short and

*Unless specifically stated otherwise, program names used are utility routines available at the NBS Computer center. 
meaningless. Reading of the data from the drum is not possible when there are errors of the first type, and at the time this part of the data manipulation was being programmed, an attempt to read a non-numeric character on a numeric format resulted in termination of the reading process and proceeding to the next executable statement. Thus, to overcome errors of type one, the invalid character must first be located and then altered to be acceptable to the computer (if not the analyst).

One way to locate the bad character is to reread the 1542 characters into a 1542 word array, and compare them against the information contained in table 56. The digits zero through nine, the alphabet, and some punctuation marks are listed in column l. Each of these characters is represented internally in the 1108, by a particular 36-bit string. The octal representation of the bit string for each character is shown in column 2, and the same "number" printed out on an integer (base ten) format is shown in column 3. Inspection of table 56 shows that valid numeric characters must be in the base ten range -17094651578 to -7430975162 (inclusive). Characters outside this range will not be the digits 0-9, and thus it is easy to detect non-numeric or good characters without testing each one to determine if it is one of the ten acceptable digits. Using this technique requires care to avoid overflows. An IF statement comparing a letter "Z" and a numeric "O" might result in the subtraction of the "O" from the "Z": i.e.

$$
\begin{array}{r}
33371214149 \\
+17094651578 \\
\hline 50465865727
\end{array}
$$

while the computer gets, incorrectly, -18253611008. To avoid this difficulty, the first test must determine whether the character is "positive" (invalid) or "negative" (valid or invalid), and the second test, on "negative" characters determines if the value is in the range indicated above. 
Table 56. Representation of alphanumeric character string internally and as base ten numbers.

\begin{tabular}{|c|c|c|}
\hline U & 600505050505 & $-170946,5157$ \\
\hline 1 & 610505050505 & -1502090975 \\
\hline 2 & 620505050505 & -14914716793 \\
\hline 3 & 630505050505 & -13873420105 \\
\hline 4 & 640505050505 & $-1 \geq 799684282$ \\
\hline 5 & 650505050505 & -11725942455 \\
\hline 6 & 660505050505 & $-1: 65220 n 63:$ \\
\hline 7 & 670505050505 & -957845881 \\
\hline$d$ & 700505050505 & $-35047169 R=$ \\
\hline o & 710505050505 & $-74.31097516 ?$ \\
\hline A & 06,0505050505 & $652766.854 ?$ \\
\hline$\sqrt{x}$ & 070505050505 & 760141.1373 \\
\hline C & 100505050505 & 3675152157 \\
\hline $\mathrm{L}^{\prime}$ & 110505050505 & $9748004 n ? 1$ \\
\hline$f$ & 120505050505 & 1,822635945 \\
\hline $\mathrm{F}$ & 130505050505 & 118963776 ng \\
\hline 1 & 140505050505 & 12970117493 \\
\hline Fi & 150505050505 & $1+043861317$ \\
\hline I & 160505050505 & 1511760.3141 \\
\hline J & 170505050505 & 10191344965 \\
\hline k & 200505050505 & 172650106789 \\
\hline L & 210505050505 & 183389.28613 \\
\hline$M_{i}$ & 2205050505015 & 194125704.37 \\
\hline 1.1 & 230505050505 & 2048631ว?6i \\
\hline 0 & 240505050505 & 21560054095 \\
\hline $\mathrm{P}$ & 250505050505 & 22633795909 \\
\hline 0 & 260505050505 & 237075.377 .33 \\
\hline R & 270505050505 & 247812795.57 \\
\hline 5 & 300505050505 & 25355021381 \\
\hline$T$ & 310505050505 & $2692876 x 203$ \\
\hline U & 320505050505 & 23002505020 \\
\hline v & 330505050505 & $2907621+6853$ \\
\hline$w$ & 340505050505 & 3.) 149988677 \\
\hline$x$ & 350505050505 & $3122.373 \cap 5 r_{i 1}$ \\
\hline Y & 360505050505 & 32297472325 \\
\hline$z$ & 3705051150505 & 33371214149 \\
\hline , & 560505050505 & -19242135225 \\
\hline & 750505050505 & -3136007866 \\
\hline+ & 420505050505 & $-3212703711^{4}$ \\
\hline I & 740505050505 & -1209743690 \\
\hline$\pi$ & 470505050505 & -26758.3279011 \\
\hline * & 500505050505 & -2568458617 \\
\hline & 510505050.505 & -24610844346 \\
\hline & & . \\
\hline
\end{tabular}


After a bad character is detected, it must be converted to some numeric character and the repaired array written on a drum so that the reading on a numeric format may continue. When an error is discovered, the magnitude of the data word (channel) in which the bad character(s) occurred is set to zero. It would be possible in some cases to estimate the number of counts in the bad channel if it occurred singly, but errors frequently have appeared in groups of successive channels, and the remedy of setting the bad channels to zero and printing out their channel numbers has been sufficient.

After the error correction part of ALSPIS was completed and debugged, the read statement which has the form $\operatorname{READ}(i, j, E R R=k, E N D=1)$ list

i = unit number

$j=$ format statement number

$k=$ a statement number

$1=$ a statement number

list $=$ the variables being set by the reading process

was modified by the NBS Computer Services Department. Originally, the clause, ERR=k, caused a transfer to statement number $\underline{k}$ in the event an error occurred in data transmission in the computer. The modification resulted in invoking the transfer whenever an attempt was made to read invalid data, such as attempting to read non-numeric characters on a numeric format. This is a very convenient feature, and the Computer Services Department is to be commended. It is unfortunate that computer manufacturers did not implement this feature long ago.

Error locating and correction using this feature would be somewhat simpler than the method described above, but it is pointless to replace that method since it is debugged and operational and errors occur infrequently. 
After the spectrum, with any corrections, has been stored in the computer core by the data manipulation part of ALSPIS, the result computation phase, part two of ALSPIS, is initiated. The Ge(Li) detector spectrum is searched for significant peaks, and their net and gross, boundary channels, full widths at half maximum, energies, and the standard deviations of the net areas are calculated and are printed. A significant peak is arbitrarily chosen as having a net area of more than 50 counts and a standard deviation of 25 percent or less. The analyst has the freedom to modify these criteria. The basic method of peak location utilizes spectrum smoothing and differentiation, which has been published and is summerized in reference [52]. Advantages of the method are speed and sensitivity. Computation time for a 4096 channel spectrum is a few seconds or less, and peaks which are quite small, relative to background, are as easily located as more prominent ones.

The major problem in computing net peak areas is in calculation of the underlying base area. The problem is trivial if the net area is large relative to the base area and there are no interfering peaks or compton edges. The top of the base is well approximated by a straight line tangent to the spectrum at either side of the peak. On the other hand, the top of the base area is probably not accurately given by a straight line when the net area is small or is small relative to the base area. The selection of the junctions of the line representing the base top with the spectrum itself may be quite difficult when an accurate net area is sought. Clearly, computation of the base area is difficult even for prominent peaks if they are not cleanly resolved from other proximate peaks.

These problems are accounted for in the peak area computations of ALSPIS without requiring appreciable amounts of computer time, and the analyst is provided with information on all peaks in the spectrum. By inspection of the results 
printed, he can determine how well (i.e. with what relative error) a net area is determined, and accept the result (which often happens), or use his own calculation method (which is often not really much better) if the peak is not well resolved or is small relative to its base. The latter choice is indicative of the need for further study to improve ALSPIS.

In operation, the peaks and boundary channels are located by finding sign changes in the smoothed first derivative [55, 56]. The net area is computed as indicated in ref. [55], and the results are printed. Boundary channels for the same peak are then re-evaluated by searching for minima in the smoothed spectrum. Using these boundary channels, a second net area for the same peak is computed and printed. Of course, the second area is different from the first only if one or both boundary channels change as a result of the re-evaluation. A second attempt at boundary modification uses the slope method [52], yielding an area computed with a third set of boundary channels. A fourth evaluation involves the regularity of the behavior of the smoothed first derivative; specifically the derivatives should approach zero monotomically as the channel number changes from half height toward the boundary channel. If it does not, the possible presence of an interfering peak is suggested. This behavior of the derivative supplies a fourth area for the same peak. A fifth way to determine peak boundary channel is to use the smoothed first derivative calculated via a different formula [57, 58] which also tends to detect interfering peaks. The last (sixth) method of peak area computation uses smoothed counts in the boundary channels for the base area computation.

Thus, areas are computed with boundary channels selected by six different methods. Usually the same boundaries are selected by several of the methods. If each of the six methods yields substantially the same net area, it is very likely that the peak is free of interference and its net area is accurately calculated for use in subsequent compu- 
tations. If poor agreement is obtained, then there is either an interfering peak or the peak area is subject to uncertainties because of its small size relative to its base. In this case, the analyst should examine the results, and he is then able to accept one of the results, compute the area himself, use a different peak from the same isotope, or use a more elaborate computer program for that particular peak. It should be noted that the accuracy of the general peak area computation has been established [56].

Part three of ALSPIS provides progress flow, guided by data control cards supplied by the analyst. These cards inform the program of various options such as the source of the data (magnetic tape or cards), and various input parameters such as energy coefficients, peak size limits, and so forth. Considerable allowance is made for improperly punched cards, and many spectra may be processed with only a few data control cards. With all parameters set, either by the program or the analyst, the data manipulation phase begins, and when complete, result computation begins. Depending on information specified by the data control cards, computation is followed by data manipulation of a new spectrum, reading of additional data control cards, or job termination.

(H. P. Yule)

C. A Mini-Computer and the Activation Analysis Section

The Activation Analysis Section recently acquired a used Varian-622/i* minicomputer with an 18 bit word size and an 8192 word memory (fig. 33). Basically, there are two ways in which we might choose to use such a computer: (I) for data acquisition and manipulation and (2) for various computations including result computation. There are many ways in which to implement these choices, especially with the addition of various peripherals such as the magnetic drum, not delivered until some time after the computer arrived, and various

* See disclaimer in Preface. 


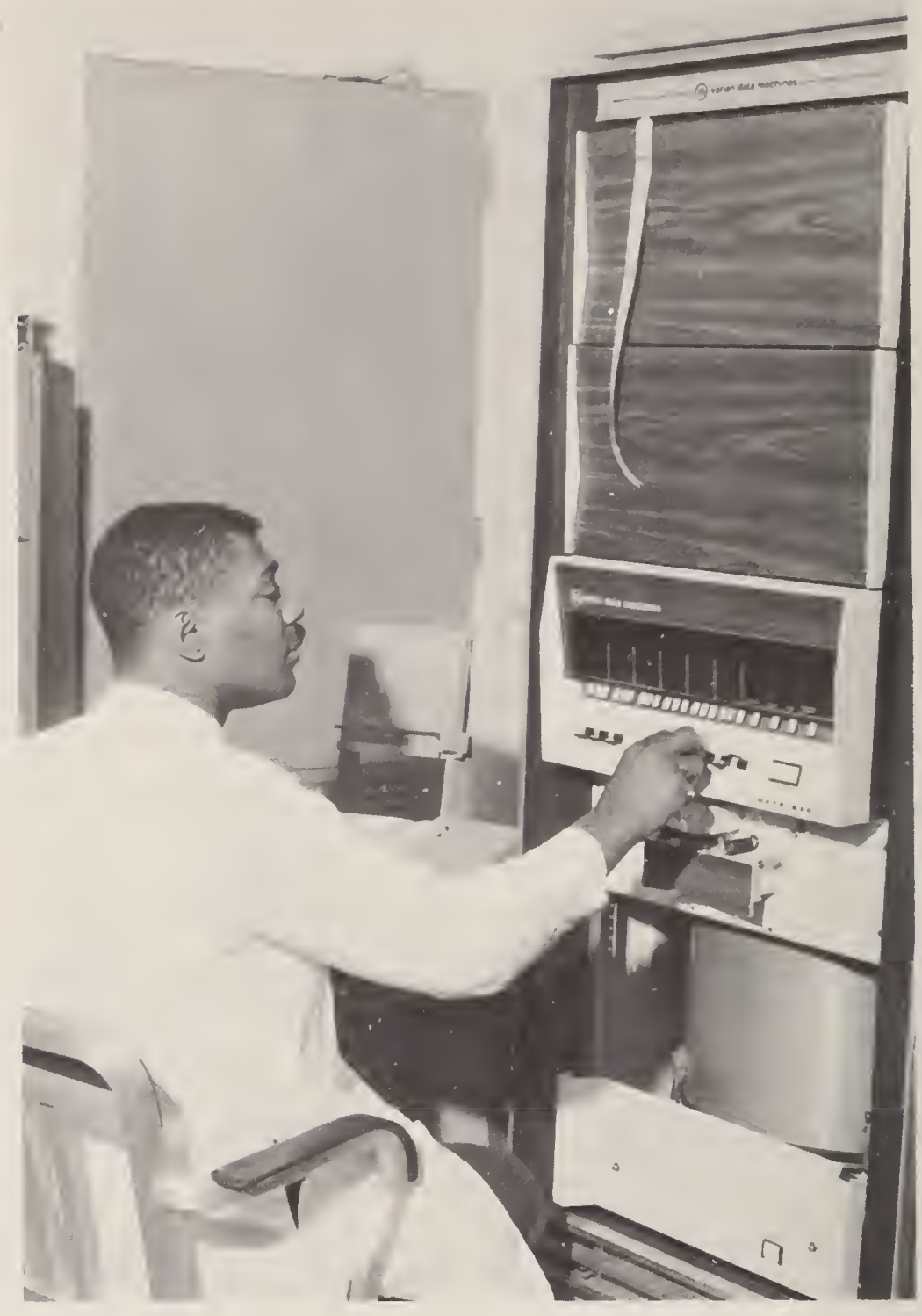

Figure 33. Varian 622/i minicomputer.

magnetic tape units. One possible way to use the computer would be to have it act as a secretary, acquiring spectral data, and storing it on magnetic tape for subsequent processing by the UNIVAC 1108. The data could also be relayed via telephone lines for remote batch processing. Alternately, the Varian could be used to perform the result computations as well as to acquire, manipulate, and store it. In this event, the printing speed of the teletype becomes a factor, 136 
and it would be possible to use the UNIVAC 1108 as a result printer, transmitting results to it either by telephone line or magnetic tape.

With these and many other analyzer-computer-peripheral configurations to be considered, the decision on the optional arrangement clearly could not be made without having a thorough knowledge of the capabilities of the Varian. A relatively near term goal, which could be sought prior to deciding on the final configuration, is to have the computer acquire data directly from the multichannel analyzers or other devices, and transcribe the data on magnetic tape for storage and processing by the UNIVAC.

What has been done, therefore, is to work with the Varian to acquire a thorough knowledge of its capabilities. In this way it has been possible to make steady progress toward several goals: (l) the near term analyzer to magnetic tape capability, (2) a decision on the final configuration of the analyzer-computer-peripheral system, (3) determination of its capabilities as a computer including hardware and software, and (4) acquisition of a knowledge of its operation also in terms of both hardware and software.

The first step in this course of action was to attend a two week course, given by Varian, to learn to operate the computer and to learn to program in the 622/i machine language and also the assembly language, DAS. The next two months were spent trying to get the machine running, since it was not operable when received and had many hardware bugs. During this period, the magnetic drum arrived, but both hardware and some of the software were of the 16 bit word variety, (Varian also manufactures the similar 620/i with 16 bit words) and Varian had to modify the drum controller board and send for new software from the factory. Debugged, the drum has a capability of storing 27,648 words, 18 bits per word. During this period, the TYMSHARE 620/i simulator was used, but it was not too helpful because of differences between 
the TYMSHARE 620/i computers ( 16 bit words, $12 \mathrm{~K}$ word memory) and our machine.

It was apparent from watching machine operation in class that the speed of the teletype (10 characters per second) as an input/output device would severly limit the rate of job throughput. In particular, the reading of punched paper tapes containing source or binary programs consumed considerable time. Therefore a high speed paper tape reader (300 characters per second) was added, effectively reducing program compilation time by roughly a factor of two. In some instances the factor of 30 could be realized - loading of the DAS assembler was reduced from roughly half an hour to one minute, for example. The reader was fully operational when installed, but no supporting software was supplied, and it was necessary to modify the paper tape loader program and the DAS assembler to read paper tapes with the high speed reader rather than the teletype.

With the system operational, a number of programs have been written to approach the goals indicated above. It is not necessary to list all these programs here, and it will be sufficient to indicate briefly the current state of sophistication. The DAS assembler, utility program, and an executive program are stored in the drum, and these programs are easily reloaded in the event of a disasterous programming error. The program to do this is called EXEC-I; it utilizes the Varian supplied drum driver and a number of routines written here. Binary to decimal and decimal to octal subroutines have been written since the Varian supplied software for these purposes is not adequate to handle our requirements. (Their binary to decimal routine is limited to non-negative numbers less than 10000, for example). Gamma ray spectra may be read from punched paper tape, converted to binary, and stored on the drum. This spectrum may be smoothed or differentiated. Peaks may be located using the smoothed first derivative, and peak areas computed using 
double precision integer arithmetic.

The next step will be to program the computer to interrupt computation, save registers and core using the drum, accept a spectrum from an analyzer, store it, and return to the original computation. In this regard, the speed of the teletype for printing results is beginning to be a severe limitation.

Further future developments will seek the goals indicated above. It is also clear at this point that a FORTRAN Compiler is needed.

(H. P. Yule)

D. Miscellaneous

A number of existing computer programs were modified and tested for use by our section. These include CLSQ, the decay curve analysis program [59], and SAMPO, the complex photopeak resolution program [53]. A study of upper limits to non-resolved components was initiated with Prof. Emile Schweikert of the Activation Analysis Research Laboratory at Texas A\&M University. A number of programs were written for our section to use with the on-line computers.

(H. P. Yule) 


\section{A. Personnel Listing}

Activation Analysis Section

Philip D. LaFleur, Section Chief

Betty E. Crowther, Secretary

Patricia Case (Student Aid)

Activation Analysis, Reactor

P. D. LaFleur, Project Leader

T. E. Gills

W. D. Kinard

H. L. Rook

J. S. Olin, Guest Worker, Smithsonian Institution

Stephanie Snead (Summer Worker)

Activation Analysis, Cockcroft-Walton Generator

S. S. Nargolwalla, Project Leader and Assistant Section Chief

J. E. Suddueth

Sheryl Birkhead (Summer Worker)

G. W. Smith (Transferred)

Activation Analysis, LINAC

G. J. Lutz, Project Leader

L. W. Masters ( $1 / 2)$

Radiochemical Separations

B. A. Thompson, Project Leader

W. F. Marlow, Guest Worker, USAEC

L.W. Masters (1/2)

Activation Analysis of Biological Materials

D. A. Becker, Project Leader

Nuclear Data Handling

H. P. Yule, Project Leader

Nuclear Track Technique

B. S. Carpenter, Project Leader 
P. D. LaFleur, "Determination of Molybdenum in Steels and Biological Materials by Activation Analysis," Radiochem. Radioanal. Letters, 1, 255, (1969).

L. T. McClendon, I. H. Qureshi, and P. D. LaFleur, "Extraction Studies of the Group IIIB-VIIB Elements and the Lanthanides Utilizing Bis(2-Ethyl-Hexyl) Orthophosphoric Acid," Radiochim. Acta, 12, 107, (1969).

B. A. Thompson and P. D. LaFleur, "Activation Analysis for Molybdenum in Samples Containing Large Amounts of Tungsten," Anal. Chem. 41, 1888, (1969).

G. J. Lutz and P. D. LaFleur, "Determination of Yttrium in Rare Earths by Photon Activation Analysis," Talanta, 16, 1457, (1970).

G. J. Lutz, "Determination of Oxygen in Sodium by Photon Activation Analysis," Anal. Chem. 42, 531, (1970).

G. J. Lutz, "Determination of Carbon in High Purity Metals by Photon Activation Analysis," Anal. Chem. 42, 948 , (1970).

S. S. Nargolwalla, M. R. Crambes and J. E. Suddueth, "Photon Self-Absorption Corrections for the Minimization of Systematic Errors in 14-MeV Neutron Activation Analysis," Anal. Chim. Acta, 49, 402, (1970).

B. S. Carpenter and C. H. Cheek, "Trace Determination of Uranium in Biological Material by Fission Track Counting," Anal. Chem. 42, 121, (1970).

G. J. Lutz, "Forensic Science: A Bibliography of Activation Analysis Papers," NBS Tech. Note 519 (1970).

G. J. Lutz, "Determination of the Light Elements in Metals: A Bibliography of Activation Analysis Papers," NBS Tech. Note $524(1970)$.

G. J. Lutz, "Pollution Analysis: A Bibliography of the Literature of Activation Analysis," NBS Tech. Note 532 (1970).

G. J. Lutz, "14-MeV Neutron Generators in Activation Analysis: A Bibliography," NBS Tech. Note 533 (1970).

G. J. Lutz, "Oceanography: A Bibliography of Selected Activation Analysis Literature," NBS Tech. Note 534 (1970). 
"Register of Personnel Working in Activation Analysis" NBS Miscellaneous publication.

P. D. LaFleur, "Laboratory of the Issue," J. of Radioanal. Chem. 3, 127 (1969).

S. S. Nargolwalla, J. Niewodniczanski, J. E. Suddueth, "Experimental Sensitivities for 3-MeV Neutron Activation Analysis," J. Radioanal. Chem. (in press).

D. A. Becker and P. D. LaFleur, "Production and Certification of NBS Biological Standard Reference Materials," Proceedings, 4 th Annual Conference on Trace Substances in Environmental Health, June 1970, Columbia, Mo. (in press).

S. S. Nargolwalla, "Application of Neutron Generators to Activation Analysis," Proceedings, Conference on Applications of Small Accelerators, Oak Ridge, Tennessee, March 1970 (in press).

\section{List of Talks}

P. D. LaFleur, "Determination of Cerium, Molybdenum and Scandium in Steel and Rock Samples Using a Radiochemical Separation and Selective Stripping," l58th National Meeting American Chemical Society, New York, New York, 8 September 1965 .

B. A. Thompson, "The Determination of Trace Elements in Glass by Activation Analysis Using Hydrated Antimony Pentoxide for Sodium Removal," (T. E. Gills, W. F. Marlow, and B. A. Thompson) 158th National Meeting, American Chemical Society, New York, New York, 8 September 1969.

D. A. Becker, "Neutron Activation Analysis for Uranium Using a Rapid Radiochemical Separation," (D. A. Becker and P. D. LaFleur) 158th National Meeting, American Chemical Society, New York, New York, 8 September 1969.

G. J. Lutz, "The Determination of Oxygen in Sodium by Photon Activation Analysis," 158th National Meeting, American Chemical Society, New York, New York, 8 September 1969.

L. W. Masters, "The Extraction of the Transition Elements Using the Antibiotic, Tetracycline," 158th National Meeting, American Chemical Society, New York, New York, 12 September 1969.

- D. A. Becker, "Neutron Activation Analysis for Sodium at the Picogram Level," American Nuclear Society, Winter Meeting, San Francisco, 2 December 1969. 
T. E. Gills, "The Determination of Short-Live Trace Impurities in Sodium Metal," (T. E. Gills and P. D. LaFleur) American Nuclear Society, Winter Meeting, San Francisco, 2 December, 1969.

B. A. Thompson, "Analysis of Medeival Stained Glass by Neutron Activation," (J. S. Olin, E. C. Miller and B. A. Thompson) American Nuclear Society, Winter Meeting, San Francisco, 2 December 1969.

P. D. LaFleur, "Novel Techniques in Neutron Activation Analysis," American Nuclear Society, Winter Meeting, San Francisco, 3 December 1969.

S. S. Nargolwalla, "Experimental Sensitivities for 3-MeV Neutron Activation Analysis," (S. S. Nargolwalla and J. E. Suddueth) American Nuclear Society, Winter Meeting, San Francisco, 4 December 1969.

G. J. Lutz, "Determination of Carbon in Metals by Photon Activation Analysis," (G. J. Lutz and L. W. Masters) American Nuclear Society, Winter Meeting, 4 December 1969.

H. L. Rook, "The Determination of Oxygen in Silicon by Charged Particle Activation Analysis," (H. L. Rook and E. A. Schweikert, Texas A\&M University) American Chemical Society Meeting, Houston, Texas, 24 February 1970.

S. S. Nargolwalla, "Advances in Neutron Generator Activation Analysis," American Chemical Society Meeting, Houston, Texas, 24 February 1970.

B. A. Thompson, "Determination of Trace Impurities in Ruby Laser Crystals by Activation Analysis," American Chemical Society Meeting, Houston, Texas, 24 February 1970.

D. A. Becker, "Characterization of a Nuclear Reactor for Neutron Activation Analysis," (D. A. Becker and P. D. LaFleur) American Chemical Society Meeting, Houston, Texas, 24 February 1970 .

G. J. Lutz, "Photonuclear Activation Analysis," American Chemical Society Meeting, Houston, Texas, 24 February 1970.

P. D. LaFleur, "Radiochemical Separations in Activation Analysis," American Chemical Society Meeting, 24 February 1970 .

P. D. LaFleur, "Trace Analysis: A Frontier of Analytical Chemistry," Seton Hall University 1969-1970 Guest Seminar Program of Eminent Scientists, South Orange, New Jersey, 17 February 1970. 
S. S. Nargolwalla, "Application of Neutron Generators to Activation Analysis," Conference on Small Accelerators for Teaching and Research, Oak Ridge Associated Universities, Oak Ridge, Tennessee, 24 March 1970.

B. S. Carpenter, "The Nuclear Track Technique, A Manual Means of Activation Analysis," Morgan state College, Baltimore 1 May 1970.

P. D. LaFleur, "Activation Analysis," Renssalaer PolyTechnic Institute, Troy, New York, 25 May 1970.

P. D. LaFleur, "Standards for Activation Analysis: Are There Any?" Joint Chemical Institute of Canada and American Chemical Society Conference, Toronto, Canada, 27 May 1970.

B. S. Carpenter, "The Nuclear Track Technique: A Growing Microanalytical Tool," Joint Chemical Institute of Canada and American Chemical Society Conference, Toronto, Canada, 28 May 1970 .

G. J. Lutz, "Determination of Carbon and Oxygen in Sodium by Photon Activation Analysis," Society of Applied Spectroscopy, 2lst Mid-America Symposium on Spectroscopy, Chicago, 3 June 1970.

D. A. Becker, "Production and Certification of NBS Biological Standard Reference Materials," (D. A. Becker and P. D. LaFleur) 4th Annual Conference on Trace Substances in Environmental Health, University of Missouri, Columbia, Missouri, 25 June 1970.

B. S. Carpenter, (paper read by L. A. Currie) "Boron and Uranium Determination in Simulated Lunar Glass by the Nuclear Track Technique," Annual Meeting of the American Nuclear Society, Los Angeles, I July 1970.

S. S. Nargolwalla, (paper read by L. A. Currie) Determination of Pulse Pile-up and Nuclear Interferences in $14-\mathrm{MeV}$ Neutron Activation Analysis for Trace Oxygen." Annual Meeting of the American Nuclear Society, Los Angeles, l July 1970. 
8. ACKNOWLEDGMENTS

The Activation Analysis Section wishes to thank Dr. Robert S. Carter, Mr. Tawfik M. Raby, Mr. James F. Torrence and the operating crews of the NBS Reactor for making the irradiation of samples possible. We also wish to acknowledge the assistance of Mr. Hugh E. DeSpain and the NBS Health Physics staff who have helped us with the safe handling of these samples. Further the assistance of Dr. J. E. Leiss and staff of the LINAC Radiation Division for arrangements to use the LINAC facilities is gratefully acknowledged.

We wish to thank the U. S. Atomic Energy Commission for making possible the visit of Mr. W. F. Marlow.

This section acknowledges Mrs. R. S. Maddock for her assistance in preparing this report.

Special appreciation is expressed to Mrs. B. Crowther who typed the entire report and whose tireless efforts as section secretary have made our entire operation more efficient and effective. 


\section{REFERENCES}

[1] LaFleur, P. D. Editor, NBS Tech. Note 508, 14 (1969).

[2] ASTM Publication E 262-65T, "Measuring Thermal Neutron Flux by Radioactivation Techniques," published by ASTM, Philadelphia, Pa. (1965).

[3] DeVoe, J.R., Editor, NBS Tech. Note 404, 14, (1966).

[4] Lafleur, P. D., Editor, NBS Tech. Note 508, 11, (1969).

[5] Euler, B. A., Covell, D. F. and Yamamoto, S., Nucl. Inst. and Meth. 72, 143 (1969).

[6] Olin, J. S. and Sayre, E. V., Proceedings. Modern Trends in Activation Analysis, NBS Special Publication 312, Vol. I, U. S. Government Printing Office, Washington, D. C., (1969).

[7] Perlman, I. and Asaro, F., Archeometry 11, 21 (1969).

[8] LaFleur, P. D., Editor, NBS Tech. Note 508, 34, (1969).

[9] Coleman, R. F. and Wood, G. A., AWRE Report No. 03/68 (April 1968).

[10] Turner, W. E. S., J. Soc. Glass Technology 40, 39 (1956); ibid. 40, 162 (1956); ibid. 40, 277 (1956).

[1] "On Divers Arts," The Treatise of Theophilus, translated by J.G. Hawthorne and C. S. Smith, University of Chicago Press, Chicago, Ill., (1963).

[12] Osmond, R. G. and Smales, A. A., Anal. Chim. Acta 10, 117 ( 1954$)$.

[13] Knight, J. D., Novey, T. B., Cannon, C. V., and Turkevich, A., Radiochemical Studies: The Fission Product edited by Coryell and Sugarman, McGraw-Hill, 1916 ( 1951$)$.

[14] Debrun, J., Barrandon, J., and Albert, Ph., Proceedings, Modern Trends in Activation Analysis, NBS Special Publication 312, Vol. II (1969).

[15] Friedlander, G., Kennedy, J., and Miller, J., Nuclear and Radiochemistry, John Wiley and Sons, New York, 95 (1966). 
[16] This report, Section 3. B., part 1 .

[17] LaFleur, P. D., Editor, NBS Tech. Note 458, 70 (1968).

[18] Girardi, F. and Sabbioni, E., J. of Radioanal. Chem. I, 1969 (1968).

[19] Smith, G. W. and Becker, D. A., "Preparation of an NBS Biological Standard Reference Material for Trace Elemental Analysis," Proceedings, Nuclear Activation Techniques in the Life Sciences, IAEA (1967).

[20] Certificate of Analysis [Provisional], Standard Reference Material 685, High Purity Gold, U. S. Dept. of Commerce, National Bureau of Standards.

[21] DeVoe, J. R., Editor, NBS Tech. Note 276, 72 (1965).

[22] Becker, D. A. and Smith, G. W., Proceedings, Modern Trends in Activation Analysis, College Station, Texas, 230 (1965).

[23] Thompson, B. A. and LaFleur, P. D., Anal. Chem. 41, 852 (1969).

[24] Thompson, B. A. and LaFleur, P. D. Anal. Chem. 41, 1889 (1969).

[25] Thompson, B. A., Proceedings, Modern Trends in Activation Analysis, NBS Special Publication 312, Vol. I, 636 (1968).

[26] LaFleur, P. D., Editor, NBS Tech. Note 508, 40 (1969).

[27] LaFleur, P. D., Editor, NBS Tech. Note 458, 41 (1968).

[28] Covell, D. F., Anal. Chem. 31, 1785 (1959).

[29] DeVoe, J. R. Editor, NBS Tech. Note 404, 42 (1966).

[30] Carpenter, B. S. and Cheek, C. H., Anal. Chem. 42, 121 (1970).

[31] Fleischer, R. L. and Price, P. B., Science, 144, 841 (1964).

[32] Taylor, J. K., Editor, NBS Tech. Note 505, 110 (1969). 
[33] LaFleur, P. D., Editor, NBS Tech. Note 508, 65 (1969).

[34] LaFleur, P. D., Editor, NBS Tech. Note 458, 9 (1968).

[35] DeVoe, J. R., Editor, NBS Tech. Note 428, 13 (1967).

[36] Nargolwalla, S. S., Trans. Am. Nucl. Soc. 12 (2), 510 (1969).

[37] Nargolwalla, S. S. Niewodniczanski, J. and Suddueth, J. E., J. Radioanal. Chem. (in press).

[38] Przybylowicz, E. P., Smith, G. W., Suddueth, J. E., and Nargolwalla, S. S., Anal. Chem. 4l, 819 (1969).

[39] Lundgren, F. A. and Nargolwalla, S. S., Anal. Chem. 40, 672 (1968).

[40] Anders, O. U. and Brichen, D. W., Anal. Chem. 36, 287 $(1965)$.

[41] Wood, D. E., Tech. Note TN-105, Kaman Nuclear, Colorado Springs, Colorado, June 1965.

[42] Hoste, J., DeSoete, D., and Speecke, A., EUR-3565E, University of Ghent, Ghent, Belgium, June 1967.

[43] Nargolwalla, S. S., Suddueth, J. E., and Rook, H. L., Trans. Am. Nucl. Soc. (in press).

[44] Gibbons, D. and Lawson, D., Proceedings, Modern Trends in Activation Analysis, College Station, Texas, 172, (1965).

[45] Delbecq, C. J., Glendenin, I. E., and Yuster, P. H., Anal. Chem. 25, 350 (1953).

[46] Ehmann, W. D. and Huizenga, J. R., Geochem. Cosmochim Acta 17, 125 (1959).

[47] Morris, D. F. C. and Killick, R. A., Talanta 4, 51 $(1960)$.

[48] Talat-Erbin, M. and Okan, S., C.N.A.E.M. - 39, Cekmece Nuclear Research Center, Istanbul, Turkey, Sep. 1966.

[49] Lyle, S. J. and Shedrikar, A. D., Anal. Chim. Acta $\underline{32}$, $575(1965)$. 
[50] Qureshi, I. H., and Meinke, W. W., Radiochim. Acta. 2, 99 (1963).

[51] Devoe, J.R., Editor, NBS Tech. Note 428, 72 (1967).

[52] See, for example, H. P. Yule, "Computation of Experimental Results in Activation Analysis," in J. R. DeVoe, Editor, Modern Trends in Activation Analysis, NBS Special Publication 312, Vol II, 1155 (1969).

[53] Routti, J. T. and Prussin, S. G., UCRL-17672.

[54] Quittner, P., Anal. Chem. 41, 1504 (1969).

[55] Yule, H. P., Anal. Chem. 38, 103 (1966).

[56] Yule, H. P., Anal. Chem 40, 1480 (1968).

[57] Heydorn, K., private communication, (1968).

[58] Barnes, V., IEEE Trans. Nucl. Soc. NS-15, 437 (1968).

[59] Cumming, J. B., BNL-6470; also NAS-NS 3107, 25 (1963). 

Latest developments in the subject area of this publication, as well as in other areas where the National Bureau of Standards is active, are reported in the NBS Technical News Bulletin. See following page. 


\section{HOW TO KEEP ABREAST OF NBS ACTIVITIES}

Your purchase of this publication indicates an interest in the research, development, technology, or service activities of the National Bureau of Standards.

The best source of current awareness in your specific area, as well as in other NBS programs of possible interest, is the TECHNICAL NEWS BULLETIN, a monthly magazine designed for engineers, chemists, physicists, research and product development managers, librarians, and company executives.

If you do not now receive the TECHNICAL NEWS BULLETIN and would like to subscribe, and/or to review some recent issues, please fill out and return the form below.

Mail to: Office of Technical Information and Publications National Bureau of Standards

Washington, D. C. 20234

Name

Affiliation

Address

City State Zip

Please send complimentary past issues of the Technical News Bulletin.

Please enter my 1-yr subscription. Enclosed is my check or money order for $\$ 3.00$ (additional $\$ 1.00$ for foreign mailing). Check is made payable to: SUPERINTENDENT OF DOCUMENTS.

TN 548 


\section{PERIODICALS}

JOURNAL OF RESEARCH reports National Bureau of Standards research and development in physics, mathematics, chemistry, and engineering. Comprehensive scientific papers give complete details of the work, including laboratory data, experimental procedures, and theoretical and mathematical analyses. Illustrated with photographs, drawings, and charts.

Published in three sections, available separately:

\section{Physics and Chemistry a.}

Papers of interest primarily to scientists working in these fields. This section covers a broad range of physical and chemical research, with major emphasis on standards of physical measurement, fundamental constants, and properties of matter. Issued six times a year. Annual subscription: Domestic, $\$ 9.50$; foreign, $\$ 11.75^{*}$.

\section{Mathematical Sciences}

Studies and compilations designed mainly for the mathematician and theoretical physicist. Topics in mathematical statistics, theory of experiment design, numerical analysis, theoretical physics and chemistry, logical design and programming of computers and computer systems. Short numerical tables. Issued quarterly. Annual subscription: Domestic, $\$ 5.00$; foreign, $\$ 6.25 *$.

\section{Engineering and Instrumentation}

Reporting results of interest chiefly to the engineer and the applied scientist. This section includes many of the new developments in instrumentation resulting from the Bureau's work in physical measurement, data processing, and development of test methods. It will also cover some of the work in acoustics, applied mechanics, building research, and cryogenic engineering. Issued quarterly. Annual subscription: Domestic, $\$ 5.00$; foreign, $\$ 6.25 *$.

\section{TECHNICAL NEWS BULLETIN}

The best single source of information concerning the Bureau's research, developmental, cooperative and publication activities, this monthly publication is designed for the industry-oriented individual whose daily work involves intimate contact with science and technology-for engineers, chemists, physicists, research managers, product-development managers, and company executives. Annual subscription: Domestic, $\$ 3.00$; foreign, $\$ 4.00 *$.

- Difference in price is due to extra cost of foreign mailing.

\section{NONPERIODICALS}

Applied Mathematics Series. Mathematical tables, manuals, and studies.

Building Science Series. Research results, test methods, and performance criteria of building materials, components, systems, and structures.

Handbooks. Recommended codes of engineering and industrial practice (including safety codes) developed in cooperation with interested industries, professional organizations, and regulatory bodies.

Special Publications. Proceedings of NBS conferences, bibliographies, annual reports, wall charts, pamphlets, etc.

Monographs. Major contributions to the technical literature on various subjects related to the Bureau's scientific and technical activities.

\section{National Standard Reference Data Series.} NSRDS provides quantitative data on the physical and chemical poperties of materials, compiled from the world's literature and critically evaluated.

Product Standards. Provide requirements for sizes, types, quality and methods for testing various industrial products. These standards are developed cooperatively with interested Government and industry groups and provide the basis for common understanding of product characteristics for both buyers and sellers. Their use is voluntary.

Technical Notes. This series consists of communications and reports (covering both other agency and NBS-sponsored work) of limited or transitory interest.

Federal Information Processing Standards Publications. This series is, the official publication within the Federal Government for information on standards adopted and promulgated under the Public Law 89-306, and Bureau of the Budget Circular A-86 entitled, Standardization of Data Elements and Codes in Data Systems. 
U.S. DEPARTMENT OF COMMERCE

WASHINGTON, D.C. 20230

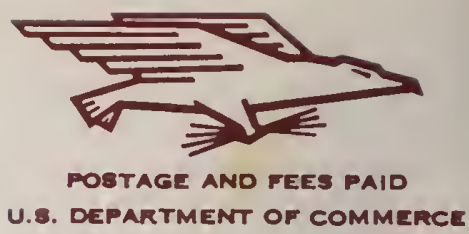

OFFICIAL BUSINESS

U.8. DEPARTMENT OF COMMERCE

PENALTY FOR PRIVATE USE, $\$ 300$ 